\title{
Comparison of Space Launch System Aerodynamic Surface Pressure Measurements from Experimental Testing and CFD
}

\author{
S. Naomi McMillin* and Patrick R. Shea ${ }^{\dagger}$ \\ NASA Langley Research Center, Hampton, VA 23681, USA \\ Derek J. Dalle ${ }^{\ddagger}$, Stuart E. Rogers ${ }^{\S}$, and Nettie H. Roozeboom ${ }^{\text {If }}$ \\ NASA Ames Research Center, Moffett Field, CA 94035, USA \\ Jamie G. Meeroff" ${ }^{\|}$and Henry C. Lee** \\ Science \& Technology Corp., Moffett Field, CA 94035, USA
}

\begin{abstract}
A comparison of surface pressure coefficient measurements obtained using pressure-sensitive paint (PSP) measurements with predictions from the computational fluid dynamics (CFD) code FUN3D is presented for the NASA SLS Block 1B crew vehicle. Overall, the flow features over the SLS configuration were captured by both the PSP data and CFD data at freestream Mach numbers $\left(M_{\infty}\right)$ of 0.8 and 1.3. Overall, the flow features over the SLS are captured by the PSP data but the intensities of large pressure gradients are less intense than what was predicted by the CFD data. Several examples of this observation are given including the flow interaction at the booster nose cone edge, core body, and forward booster attachment hardware at $M_{\infty}=0.8$.
\end{abstract}

\section{Nomenclature}

$\begin{array}{ll}C_{P} & =\text { surface pressure coefficient, } 2\left(P-P_{\infty}\right) /\left(\rho_{\infty} U_{\infty}^{2} S\right) \\ C_{P, C F D} & =\text { surface pressure coefficient from CFD study } \\ C_{P, P S P} & =\text { surface pressure coefficient from PSP measurements } \\ M_{\infty} & =\text { freestream Mach number } \\ P & =\text { local pressure, } \mathrm{lb} / \mathrm{ft}^{2} \\ P_{\infty} & =\text { freestream pressure, } \mathrm{lb} / \mathrm{ft}^{2} \\ R e & =\text { unit Reynolds number, } / \mathrm{ft} \\ S & =\text { reference area, } \mathrm{ft}^{2} \\ U_{\infty} & =\text { freestream velocity, } \mathrm{ft} / \mathrm{sec}\end{array}$

\section{Symbols}

$\alpha_{V}=$ angle of attack in the vertical-plane axis system, deg

$\beta_{V} \quad=$ sideslip angle in the vertical-plane axis system, deg

$\Delta C_{P}=$ surface pressure coefficient delta, $C_{P, C F D}-C_{P, P S P}$

$\phi_{V}=$ roll angle in the vertical-plane axis system

$\rho_{\infty} \quad=$ freestream density, $\mathrm{lbm} / \mathrm{ft}^{3}$

\footnotetext{
*Research Aerospace Engineer, Configuration Aerodynamics Branch, Mail Stop 499, AIAA Senior Member.

${ }^{\dagger}$ Research Aerospace Engineer, Configuration Aerodynamics Branch, Mail Stop 499, AIAA Member.

${ }^{\ddagger}$ Research Scientist/Engineer, Computational Aerosciences Branch, AIAA Member.

$\S$ Aerospace Engineer, Computational Aerosciences Branch, AIAA Associate Fellow.

IAerospace Engineer, Experimental Aero-Physics Branch, Mail Stop 260-1, AIAA Member.

"Research Scientist/Engineer, Computational Aerosciences Branch, AIAA Member.

**Research Scientist, Computational Aerosciences Branch, AIAA Member.
} 


\section{Acronyms}

$\begin{array}{ll}\text { ATT } & =\text { Aerodynamics Task Team } \\ \text { CFD } & =\text { computational fluid dynamics } \\ \text { ESP } & =\text { electronically scanned pressure } \\ \text { EUS } & =\text { exploration upper stage } \\ \text { GOX } & =\text { gaseous oxygen } \\ \text { LAS } & =\text { launch abort system } \\ \text { LOX } & =\text { liquid oxygen } \\ \text { MPCV } & =\text { multi-purpose crew vehicle } \\ \text { OML } & =\text { outer mold line } \\ \text { PSP } & =\text { pressure-sensitive paint } \\ \text { RANS } & =\text { Reynolds-averaged Navier Stokes } \\ \text { SLS } & =\text { Space Launch System } \\ \text { SRB } & =\text { solid rocket booster } \\ \text { TVC } & =\text { thrust vector control } \\ \text { UPWT } & =\text { Unitary Plan Wind Tunnel }\end{array}$

\section{Introduction}

$\mathrm{T}$ HE Space Launch System (SLS) is a launch platform being developed by NASA to extend human exploration beyond Earth's orbit. The SLS vehicle, seen in Fig. 1, is comprised of a liquid fuel core stage with two solid rocket boosters (SRB) common to all of the vehicle configurations. A number of upper stage vehicle components can be integrated with the core stage depending on mission requirements including the exploration upper stage (EUS) and the Orion multi-purpose crew vehicle (MPCV) for crewed missions which are part of the fully integrated Block 1B Crew Vehicle.

In support of vehicle development and characterization, the SLS Aerodynamics Task Team (ATT) is responsible for providing databases consisting of but not limited to configuration forces and moments, surface pressures, line loads, protuberance aerodynamic loads, nozzle hinge moments, base pressures, force and moment partials, unsteady buffet loads. These databases are generated on the launch vehicles throughout the entire launch from lift-off until the vehicle leaves the atmosphere. The ATT uses several tools to produce these products: wind-tunnel tests in three different wind-tunnel facilities, multiple computational fluid dynamics (CFD) computer codes, and three separate CFD groups working on different products.

A key element of the force and moment databases exists in the transonic flight regime when the flow physics can change rapidly. Thus a wind-tunnel test was conducted at the NASA Ames Unitary Plan Wind Tunnel (UPWT) facility on the SLS Block 1B crew vehicle from a freestream Mach number $\left(M_{\infty}\right)$ of 0.5 to 2.5. This test was performed on a high-fidelity, $1.3 \%$ scale model obtaining force and moment data, surface pressure data, and schlieren data. Measurements were also acquired using pressure-sensitive paint (PSP) to obtain surface pressures at higher spatial resolution than was possible with static pressure taps. Concurrent to the wind-tunnel test, computational simulations were performed using Reynolds-averaged Navier Stokes (RANS) modeling at wind-tunnel test conditions. The computational simulations were performed on the same outer mold lines (OML) as the wind-tunnel model.

In recent years, PSP techniques have matured, and although the ATT has not utilized PSP as a data source for any of its databases yet, the team is examining the viability (or accuracy) of using PSP for this purpose. With the right validation/verification regimen, PSP could be used to validate CFD and as a data source for database nominals or uncertainties for: surface pressures, protuberance aerodynamic loads, line loads, and possibly buffet loads.

The goal of the current work is to assess PSP by performing direct comparisons with one of the CFD tools that has already been used to produce multiple aero ascent databases on the present Block 1B crew vehicle and other related vehicles. Two related papers will provide detailed comparisons between the wind-tunnel test and CFD simulations focused on force and moment measurements [1] and sectional loads [2].

\section{Model Description}

The SLS 1.3\% Ascent Force and Moment Wind-tunnel Model is a 1.3\%-scale model of the full-scale SLS geometry designed with a specific focus on acquiring force and moment data in the transonic flow regime. The test hardware was supported in the wind-tunnel test section using a traditional sting-mount, and was designed in a modular fashion 
to accommodate testing of the three SLS configurations of interest. Fig. 2 contains sketches of the three wind-tunnel configurations tested. The three configurations shared a common stack (Fig. 2a) that remained attached to the sting/balance interface throughout the course of testing. The common stack consisted of the SRBs and the core body aft of the EUS. The SLS-10k and SLS-28k configurations (Fig. 2b and 2c, respectively) shared the MPCV and launch abort system (LAS) tower hardware. Similarly, the SLS-28k and SLS-27k configurations (Fig. 2c and 2d, respectively) shared the EUS hardware.

The SLS 1.3\% Ascent Force and Moment Wind-tunnel Model is based on the most up-to-date flight OML definition at the time of the model design. The SLS-10008 flight OML was used to develop the LAS tower, MPCV, SRBs, and the core stage aft of the interim cryogenic propulsion stage. The SLS-28004 geometry was the official SLS-28k flight OML during model design, but the SLS-28005 geometry was nearing completion. The EUS and the universal stage adapter were based on the SLS-28004 OML with modifications for the anticipated changes in the SLS-28005.

Post-test comparisons indicate that the major differences between the SLS-27005 and SLS-28005 wind-tunnel geometries and the official SLS-27005 and SLS-28005 geometries included standard simplifications and modifications to the geometry for scaled fabrication and the clocking angle of the cameras on the EUS. As such, the wind-tunnel configurations most closely represent the SLS-10008, the SLS-27005, and the SLS-28005 flight OMLs. For the purposes of this document, these geometries will be referred to as the SLS-10k, the SLS-27k, and the SLS-28k, respectively.

A significant number of protuberances were included as part of the model design, and a number of these protuberances were removable to allow for clean test configurations. A list of the model protuberances can be found in Table 1 .

\section{Wind Tunnel Test Description}

The experiment was conducted in the NASA Ames UPWT, which is comprised of two interconnected wind tunnels that share a main drive [3] [4] [5]. The two wind tunnels are the UPWT 11- by 11-Foot Transonic Wind Tunnel and the UPWT 9- by 7-Foot Supersonic Wind Tunnel. Both were utilized in the current test effort. Table 2 contains the flow and model conditions at which the test was conducted. Ref. [1] contains a detailed description of the test set-up. Details relevant to the PSP portion of the wind-tunnel test are described in the subsequent section.

The test model was sting mounted. Model positioning was accomplished through the use of the angle resolvers in the knuckle-sleeve adapters and the large model roll mechanism. The angle of attack $\left(\alpha_{V}\right)$, sideslip angle $\left(\beta_{V}\right)$, and roll angle $\left(\phi_{V}\right)$ conditions of the PSP portion of the test are defined in the vertical plane (i.e., the AIAA standard missile axis system) as shown in Fig. 3 [6].

The boundary layer on each of the three SLS geometries was tripped to force turbulent boundary layer development. Trip devices for SLS ascent testing are traditionally placed as rings at key axial stations, and the locations of these rings can be seen in Fig. 4. Table 3 provides the details of the trip dot heights for each wind tunnel.

A total of 42 static surface pressure taps were located at six axial locations on the test model as seen for the SLS-28k configuration in Fig. 5. At each axial station along the model, the surface pressure taps were located in rings at the radial stations defined in Fig. 6. Twenty-six of the pressure taps were located on the core body of the common stack (axial stations 3-6 identified in Fig. 5) and were connected to an internal electronically scanned pressure (ESP) measurement module throughout the duration of the test. The remaining 16 pressure taps were located on the model forebodies (axial stations 1 and 2 identified in Fig. 5) and were connected via quick disconnects to allow for more efficient model changes. A single 64 port, 15 psi ESP module was internally mounted in the common stack forward of the balance block to accommodate the surface pressure measurements. A series of check loads were performed during model build-up with and without the ESP cabling bridging the internal balance to ensure that there was no interference.

The surface pressure taps were used in the processing of the PSP data by providing pressure measurements to anchor the PSP data as discussed in the following section. Several of the tap locations were requested by the PSP team to provide a large range of pressure measurements for paint calibration. For example, the pressure station 1 taps on the forward facing (compression) surface of the model forebody (see Fig. 5) were expected to have higher pressure measurement than elsewhere on the model. Likewise, the pressure station 4 taps aft of the forward booster attachment hardware (see Fig. 5) in the expansion flow region were expected to have lower pressure measurements than elsewhere on the model.

The PSP data measurements required three coats of paint on the model. The epoxy base coat was painted in the model preparation area prior to model installation and remained on the model for the duration of force and moment testing in both tunnels. The PSP base and top coats of paint were applied in the 11- by 11-foot test section prior to commencing PSP measurements. The PSP base and top coats of paint were applied over the boundary layer trip dots following the NASA Ames UPWT best practices. Eight cameras and 40 lamps were mounted around the wind tunnel 
test section. Two cameras and 10 lamps were mounted on each wall of the test section to achieve complete coverage of the wind tunnel model at all test conditions, and to uniformly distribute the lighting. This coverage accommodated all three wind tunnel model configurations tested, removing the need to relocate any equipment during the test campaign. Fig. 7 shows four views of the lamps and cameras set up in the slotted side windows of the test section for the SLS-28k configuration at $\alpha_{V}=0^{\circ}$ and $\phi_{V}=45^{\circ}$. More details regarding the NASA Ames UPWT best practices for steady PSP can be found in the work by Roozeboom and Baerny [7].

\section{PSP Data Acquisition and Processing}

Static surface pressure tap data and pressure-sensitive paint image data were obtained simultaneously in the UPWT 11- by 11-Foot Transonic Wind Tunnel at $0.80 \leq M_{\infty} \leq 1.30$ for all three configurations. This section discusses the process used to convert the PSP image data into PSP surface pressure data.

\section{A. PSP Surface Grid}

The wind-tunnel model CAD geometry was used to generate a surface grid for the PSP data generation process. The PSP surface grid was generated separately from the CFD surface grid which is discussed in the subsequent section. Table 4 contains three parameters (patches, nodes, and cells) for both surface grids. Fig. 8 shows the patches of the upper surface for the PSP surface grid. Fig. 9 shows a close-up view of the lower SLS-28k surface grid near the forward starboard SRB attachment point. The lower left inset shows the SLS-28k surface with a red box outlining the location of the surface grid shown in Fig. 9. The PSP surface grid was generated to optimize the camera to surface resolution. Generally, at least three to five pixels were given to a surface grid cell. Of course, this is not constant as the features of the model change and the grid resolution needs to change in order to properly define certain smaller model features (e.g., cameras, liquid oxygen (LOX) lines). Fig. 10 shows an example of how pixel-to-cell resolution changed with changing cell size. The yellow lines in Fig. 10 are the edges of cells on the surface of the SLS-28k configuration. The intersections of the yellow lines are the nodes of the PSP surface grid. The smaller squares of varying gray colors are the pixels of the PSP camera. The varying gray colors are varying intensities of the PSP under flow conditions in the wind-tunnel test section.

\section{B. PSP Image Processing}

The process for converting PSP images from the camera to PSP surface pressure coefficient $\left(C_{P, P S P}\right)$ data is given in Table 5. The first step is the generation of the surface grid, which occurs once for a given model configuration. Steps 2 through 5 occur at each wind-tunnel test data point for a given condition. When a PSP data point was taken, each camera recorded the intensity the pressure-sensitive paint responding to the surface pressure exerted on the surface by the flow structures occurring over the model. The second step in the image processing maps the camera image data onto the PSP surface grid nodes. This step averaged the image data for portions of the model observed by more than one camera. It also averaged the pixels surrounding a surface grid node based on distance from the node. The next three steps convert the image intensity data to surface pressure data. Step 3 uses a lab calibration obtained using a coupon of PSP data ahead of the test. Step 4 then uses an in situ calibration (i.e., 'pump down' images). Images are taken at several static pressure (wind off) conditions to record the response of the PSP and account for nonuniformities in lighting and paint application. These images were generally obtained before the start of wind-on testing. Step 5 is a calibration using the pressure tap data and applies an overall offset to the data. This is not a local offset. It is global depending on the PSP/tap deltas. More details on the image processing is given in Ref. [7].

\section{Computational Study Description}

The wind-tunnel model CAD geometry was used to generate a surface grid for the CFD study. Table 4 contains three parameters for the CFD surface grid as well as the PSP surface grid, as mentioned earlier. Fig. 11 shows the patches of the upper surface for the CFD surface grid and can be compared to the same view of the PSP surface grid of Fig. 8 . Fig. 12 shows a close-up view of the lower SLS-28k surface grid near the forward starboard SRB attachment point. The lower left inset shows the SLS-28k surface with a red box outlining the location of the surface grid shown in Fig. 12. Fig. 9 shows the same view for the PSP surface grid. These figures show that the CFD surface grid is much finer than the PSP surface grid.

CFD surface pressure data $\left(C_{P, C F D}\right)$ are extracted from simulations of the wind tunnel geometry without wind tunnel 
walls using version 13.1 of FUN3D [8]. FUN3D is a node-based flow solver on 3D unstructured (mixed-element) meshes developed at the NASA Langley Research Center. Best practices from previous work on similar geometries were utilized in setting up the computational study. For this work, the simulations were run in RANS or time-accurate unsteady uRANS (whenever a steady-state RANS solution was not steady) mode using the Spalart-Allmaras turbulence model. Two feature-based adaptations were conducted during every CFD simulation, which increased the size of the volume mesh and helped to resolve sharp features such as shocks. The surface mesh contains $1.45 \times 10^{6}$ triangles, and the initial volume mesh contains $32.6 \times 10^{6}$ nodes and $89.4 \times 10^{6}$ cells (either triangular prism cells, pyramids, or tetrahedra). With mesh adaptation, the volume mesh is allowed to grow to $56.8 \times 10^{6}$ nodes and $228.3 \times 10^{6}$ cells.

\section{Comparison Process}

Developing a numerical comparison of the PSP data to CFD data was the primary objective of this paper. The process developed is presented in Table 6. Steps 2, 4, 5, and 6 utilized Tecplot - a data visualization and CFD post-processing tool used to discover, analyze, and communicate complex data from numerical simulations.

The first step in the comparison process was to determine the patches on the PSP surface grid that did not have PSP data mapped from the wind tunnel measurements. Most of these no-data patches were identified in a list provided by the PSP measurement team but there were other patches set to a null value in the data files. All of the no-data patches are ignored in the comparison process.

The remaining steps in the comparison process utilized Tecplot. The second step in the comparison process used the plotting feature of Tecplot to examine $C_{P, P S P}$ data to find anomalous patches. An anomalous patch was identified as one with PSP data that did not fit in with surrounding data. Fig. 13 shows $C_{P, P S P}$ data on the inboard side of the left SRB at $M_{\infty}=0.8, \alpha_{V}=0^{\circ}, \phi_{V}=0^{\circ}$. The gray patches are those identified in step 1. The patches with blue arrows pointing at them have $C_{P, P S P}$ data that do not fit in with the surrounding patches' $C_{P, P S P}$ values. These anomalous patches should be set as no-data patches.

The third step of the comparison process was to prepare the surface grid for the interpolation process by combining patches. The interpolation process of Tecplot interpolates onto a surface one patch at a time. Since the patches of the two surface grids were not identical (see Fig. 8 and 11), at times more than one CFD surface grid patch would be needed to interpolate onto the entire surface of one PSP surface grid patch. Fig. 14 shows the PSP and CFD patches for a portion of the LAS/MPCV components of the SLS-28k configuration. The yellow and bright blue patches of Fig. 14b are both needed to interpolate onto the PSP red patch in the middle of Fig. 14a. Thus, the patches of the CFD surface grid were combined into 5 large patches defining the following areas: LAS/MPCV, core, left SRB, right SRB, and sting as seen in Fig. 15. This cuts down on mapping the CFD patches to the correct PSP patches and eliminated the cases where more than one CFD patch mapped to the same PSP patch.

Steps 4-6 of the comparison process used different features within Tecplot. Step four used the interpolation feature of Tecplot to impose the CFD solution onto each patch of the PSP surface grid. Step 5 used the equations feature of Tecplot to calculate the surface pressure coefficient delta $\left(\Delta C_{P}\right)$ data, where $\Delta C_{P}=C_{P, C F D}-C_{P, P S P}$. Tecplot was then used in the final step of the comparison to generate contour plots of $\Delta C_{P}$ for analysis.

\section{Results}

Steady PSP measurements were acquired in the UPWT 11- by 11-Foot Transonic Wind Tunnel at $0.80 \leq M_{\infty} \leq 1.30$ for all three SLS configurations. This paper will present results on a smaller subset shown in Table 7 for the SLS-28k configuration. The following results will examine the $M_{\infty}=0.8$ and 1.3 data sets separately. The first section will discuss the baseline case of the SLS-28k configuration at $M_{\infty}=0.8, \alpha_{V}=8^{\circ}$, and $\phi_{V}=0^{\circ}$ by examining three different areas of the SLS-28k configuration. The second section will discuss the $M_{\infty}=1.3$ data by examining the effects of $\alpha_{V}$ and $\phi_{V}$. All data points are at $\beta_{V}=0^{\circ}$.

\section{A. Baseline Case at $M_{\infty}=0.8$}

The baseline case for the present investigation is the SLS-28k configuration at $M_{\infty}=0.8, \alpha_{V}=8^{\circ}$, and $\phi_{V}=0^{\circ}$. Fig. 16 shows $C_{P, P S P}, C_{P, C F D}$, and $\Delta C_{P}$ data for this baseline case. Fig. 16a shows three sets of color contour data for the upper surface of the SLS-23k: $C_{P, P S P}$ data are in the top image, $C_{P, C F D}$ data are in the middle image, and $\Delta C_{P}$ data are in the bottom image. The same set of images for the lower surface of the SLS-28k configuration is shown in Fig. 16b. All of the numerical values on the contour color scales have been removed except for the zero condition due to program considerations. The minimum and maximum values of the scales are not the actual minimum/maximum values of the 
data but were chosen to highlight the flow features evident in the data. The $C_{P}$ and $\Delta C_{P}$ scales are constant throughout the paper. The grey patches on the SLS-28k surface in Fig. 16 and subsequent figures are patches of the PSP surface grid where PSP data were not obtained in the wind tunnel test. Three areas on the SLS-28k configuration are highlighted by red boxes in the $\Delta C_{P}$ data of Fig. 16. These areas are identified as the following: the LAS/MPCV section, the forward SRB section, and the aft section. This section discusses the PSP results for each section on the baseline case.

\section{Aft Section}

Fig. 17 shows $C_{P, P S P}, C_{P, C F D}$, and $\Delta C_{P}$ data on the aft section of the SLS-28k configuration at $M_{\infty}=0.8, \alpha_{V}=8^{\circ}$, and $\phi_{V}=0^{\circ}$. The top left image of Fig. 17a is a color contour plot of $C_{P, P S P}$ for the upper surface and the lower left image is a contour plot of $C_{P, C F D}$. On the right of Fig. 17a is a contour plot of $\Delta C_{P}$. The icon image at the top middle of Fig. 17a has a red box showing the location of the data on the SLS-28k configuration, which for this figure is the aft section. The same set of data is presented for the lower surface in Fig. 17b.

The $\Delta C_{P}$ data of Fig. 17 show that the PSP measurements have good agreement with the CFD data with some disagreement occurring at protuberance sites. An extreme example is the aft booster attachment ring but also includes the smaller structural rings that occur along the length of the core and SRBs. Fig. 18 shows the geometry of these rings on the lower surface of the port SRB. Fig. 19 shows the $C_{P, P S P}, C_{P, C F D}$, and $\Delta C_{P}$ data at this same view for $M_{\infty}=0.8$, $\alpha_{V}=8^{\circ}$, and $\phi_{V}=0^{\circ}$. The $C_{P, C F D}$ data of Fig. 19 show a larger compression in surface pressure occurring on the forward facing step of the aft booster attachment ring than measured by PSP. The resultant $\Delta C_{P}$ data for the forward facing step of the attachment ring are positive (right side of Fig. 19). The $C_{P, C F D}$ data also show a larger expansion on the top of the ring than measured by PSP. This results in a negative $\Delta C_{P}$ on the top of the aft booster attachment ring.

The above $\Delta C_{P}$ results occur to a lesser degree on the structural ring noted in Fig. 19. The difference between the $C_{P, P S P}$ and $C_{P, C F D}$ contour data on the left of Fig. 19 are harder to grasp visually about the smaller structural ring but the $\Delta C_{P}$ data on the right of Fig. 19 clearly show the same trends as observed for the aft booster attachment ring. The same observation is true for the data about both rings at the lower $\alpha_{V}=0^{\circ}$ shown in Fig. 20.

\section{Forward SRB Section}

Fig. 21 shows $C_{P, P S P}, C_{P, C F D}$, and $\Delta C_{P}$ data on the starboard forward SRB at $M_{\infty}=0.8, \alpha_{V}=8^{\circ}$, and $\phi_{V}=0^{\circ}$. The flow feature that jumps out from the upper surface data is the sweep of negative $C_{P, C F D}$ contours from the inboard edge of the nose cone (lower left image in Fig. 21a). This feature is most likely a footprint of a flow interaction occurring between the inboard edge of the SRB nose cone, the core body, and the forward SRB attachment hardware. The origin of this interaction is just ahead of the attachment hardware as seen in the inboard-side view of the starboard SRB shown in Fig. 22. CFD predicts a stronger SRB/core/attachment interaction than was measured by PSP (upper left image of Fig. 21a). Thus, the resultant negative $\Delta C_{P}$ data are a dark blue streak angling outboard and downstream from the interaction site (right image of Fig. 21a). The above flow feature is also present on the port SRB as seen in the expanded view of the forward SRB section in Fig. 23. The expanded view of the forward SRB data has been rotated in Fig. 23 (when compared to the view in Fig. 21) to get the best magnification factor for the figure. The upper surface data of Fig. 23a show symmetric flow over and between the forward port and starboard SRBs.

Another area where the CFD predicts a larger absolute value of $C_{P}$ presence than that measured by PSP is the tip of the SRB nose cones as seen in the lower surface data of Fig. 21b. This result is also seen at the tip of the LOX line fairings as shown in the expanded view of the lower surface in Fig. 23. The expanded view of the forward SRB data has been rotated in Fig. 23 (when compared to the view in Fig. 21) to get the best magnification factor for the figure. The upper and lower LOX line fairings are labelled in Fig. 23.

A third area to note in the forward SRB data is at the SRB nose cone edge (as labelled in Fig. 21). The lower surface data in Fig. $21 \mathrm{~b}$ show a positive $C_{P, C F D}$ right up to the cone edge. In contrast, $C_{P, P S P}$ rapidly decreases just upstream of the cone edge. Thus, a strong positive $\Delta C_{P}$ value (the red contours in the right image of Fig. 21b) lies just ahead of the cone edge. This observation occurs to a lesser degree on the upper surface as noted in Fig. 21a. This observation is an $\alpha_{V}$ effect since the $\alpha_{V}=0^{\circ}$ data of Fig. 24 show that the $\Delta C_{P}$ data just upstream of the cone edge is constant around the circumference of the nose cone.

\section{3. $L A S / M P C V$ Section}

Fig. 25 shows $C_{P, P S P}, C_{P, C F D}$, and $\triangle C_{P}$ data on the MPCV of the SLS-28k configuration at $M_{\infty}=0.8, \alpha_{V}=8^{\circ}$, and $\phi_{V}=0^{\circ}$. Overall, PSP and CFD agree well on this portion of the SLS-28k configuration. There are two areas on the 
LAS tower where this observation is not the case: the LAS nozzle and the LAS nose. On the forward facing surfaces of the LAS nozzle, CFD predicts a more positive $C_{P}$ than was measured by PSP. This trend is clearly evident in the front view of the data as shown in Fig. 26. The $C_{P, C F D}$ data are hitting the maximum limit of the contour map on the forward facing surfaces of the LAS nozzle whereas as the $C_{P, P S P}$ data are much lower in value. The resultant $\Delta C_{P}$ data for the forward facing portions of the nozzle are positive. CFD also predicts a more negative $C_{P}$ than was measured by PSP for the edge of the nozzle ring and the nozzle lip. The resultant $\Delta C_{P}$ data for these edges on the nozzle are negative.

The second exception to the good agreement of CFD and PSP data is on the LAS nose - especially at the tip of the nose and at slope changes on the nose cone. Fig. 26 shows that CFD predicts a larger positive $C_{P}$ on the LAS nose tip than was measured by PSP. This observation results in a high positive $\Delta C_{P}$ for the tip of the nose. Fig. 27 shows a close-up view of the surface pressure data sets on the lower surface of the LAS nose. At each slope change labelled in the right image of Fig. 27, the $\Delta C_{P}$ data show a blue ring of negative $\Delta C_{P}$. These blue rings of negative $\Delta C_{P}$ are also evident in the front view of the data in Fig. 26. CFD predicts a greater expansion at these surface slope changes than was measured by PSP. The LAS nose and nozzles are very small compared to the total size of the SLS-28k configuration (see the icon image at the top of Fig. 27) and thus, the PSP technique may be hitting a resolution limit for capturing these data.

\section{B. $M_{\infty}=1.3$ Data}

Results for $M_{\infty}=1.3$ will only focus on the SRBs. A brief comparison with the baseline case at $M_{\infty}=0.8, \alpha_{V}=8^{\circ}$, and $\phi_{V}=0^{\circ}$ will be given. The effects of $\alpha_{V}$ and $\phi_{V}$ will then be discussed separately.

\section{Effects of $\alpha_{V}$}

Fig. 28 shows $C_{P, P S P}, C_{P, C F D}$, and $\triangle C_{P}$ data on the both SRBs near the forward SRB attachment point at $M_{\infty}=1.3$, $\alpha_{V}=8^{\circ}$, and $\phi_{V}=0^{\circ}$. The CFD data show that the flow feature at the intersection of the SRB nose cone, core, and SRB attachment hardware noted in Fig. 21a for $M_{\infty}=0.8$ is not present on the upper surface of either SRB. However, the following features also occur in the $M_{\infty}=1.3$ data:

- CFD predicts a larger positive $C_{P}$ than that measured by PSP at the SRB nose tip

- PSP measures a rapid expansion just ahead of the nose cone edge whereas CFD predicts a positive $C_{P}$ up to the nose cone edge

- PSP measures a lower absolute value of the $C_{P}$ ahead and aft of the first SRB structural ring than that predicted by CFD

At $M_{\infty}=1.3$, both PSP and CFD data captured diagonal shock patterns in the $C_{P}$ contours on the lower surface emanating from the intersection of the SRB nose cone, core, and the SRB attachment hardware as seen in the two images on the left side of Fig. 28b. The $C_{P}$ contours angle outboard and downstream from the inboard edges of the SRBs. These diagonal shock patterns are symmetric between the SRBs. The $C_{P, P S P}$ data also show another shock pattern beginning behind the first structural ring of the SRB. Actually, Fig. 29 shows this shock pattern exists at each structural ring on the lower surface of the SRB at $M_{\infty}=1.3, \alpha_{V}=0^{\circ}, 2^{\circ}, 4^{\circ}$, and $8^{\circ}$, and $\phi_{V}=0^{\circ}$. The shock patterns are visually faint in the $C_{P, P S P}$ and $C_{P, C F D}$ data at $\alpha_{V}=0^{\circ}$ (Fig. 29a) but grow more distinct with increasing angle of attack (Fig. 29b - 29d). The $\Delta C_{P}$ data of Fig. 29 do not show any signs of the shock patterns indicating that PSP measurements agree very well with the CFD predictions.

The corresponding upper surface data of Fig. 29 are presented in Fig. 30. The upper surface PSP and CFD data show similar shock patterns behind the structural rings occurring at $\alpha_{V}=8^{\circ}$ (see Fig. 30d) up to structural ring 7 on the SRB. For structural ring 7 and subsequent rings, the upper surface flow at $\alpha_{V}=8^{\circ}$ shows more than one shock pattern occurring between structural rings indicating a more complex flow developing that far aft on the SRB. For $\alpha_{V}=0^{\circ}, 2^{\circ}$, and $4^{\circ}$, the contour sweeps are very faint (see Fig. 30a - 30c) down the length of the SRB. The $\Delta C_{P}$ data of Fig. 29 do not show any signs of the sweeping shock patterns for the SRB above structural ring 7 indicating good agreement between PSP and CFD for that portion of the SRB.

\section{Effects of $\phi_{V}$}

Fig. 31 shows surface pressure data for the upper surface on both SRBs at $M_{\infty}=1.3, \alpha_{V}=8^{\circ}$, and $\phi_{V}=0^{\circ}, 45^{\circ}$, and $90^{\circ}$. $C_{P, P S P}, C_{P, C F D}$, and $\Delta C_{P}$ data are shown in the images going from left to right, respectively, for each part of Fig. 31 . The SLS-28k configuration is rolled in the clockwise direction for positive $\phi_{V}$ - meaning the starboard side is the windward 
side for $\phi_{V}=90^{\circ}$. The symmetrical shock patterns evident in the $C_{P, P S P}$ and $C_{P, C F D}$ data for $\phi_{V}=0^{\circ}$ (Fig. 31a) are skewed as $\phi_{V}$ increases (Fig. 31b and 31c). Additionally, new shock patterns now emerge from the fairing and brackets of the upper LOX line as as $\phi_{V}$ increases. The CFD and PSP results are in good agreement as the data agree on the effect of $\phi_{V}$ on the upper surface as indicated by the $\Delta C_{P}$ data of Fig. 31. The same trends observed on the upper surface also appear in the lower surface data similarly presented in Fig. 32.

\section{Conclusions}

In order to support the NASA SLS program, the aerodynamics task team is conducting wind-tunnel experiments to aid in developing force and moment databases. A force and moment wind-tunnel test was conducted at the NASA Ames Unitary Plan Wind Tunnel facility on the SLS Block 1B crew vehicle from $0.5 \leq M_{\infty} \leq 2.5$. Measurements were also acquired using pressure-sensitive paint (PSP) to obtain surface pressures at higher spatial resolution than possible with static pressure taps. Concurrent to the wind-tunnel test, computational simulations were performed using RANS modeling at wind-tunnel test conditions. The computational simulations were performed on the same outer mold lines as the wind-tunnel model.

A comparison process was developed to compare the PSP surface pressure coefficient $\left(C_{P, P S P}\right)$ measurements with CFD predicted surface pressure coefficient $\left(C_{P, C F D}\right)$ data. The process heavily utilized the data visualization and manipulation computer code Tecplot. The end result is the delta $C_{P}$ parameter on the PSP surface mesh, where $\Delta C_{P}=C_{P, C F D}-C_{P, P S P}$. The process was applied to 20 cases on the SLS-28k configuration varying in freestream Mach number $\left(M_{\infty}=0.8\right.$ and 1.3$)$, angle of attack $\left(\alpha_{V}=0^{\circ}, 2^{\circ}, 4^{\circ}\right.$, and $\left.8^{\circ}\right)$, and roll angle $\left(\phi_{V}=0^{\circ}, 45^{\circ}\right.$, and $\left.90^{\circ}\right)$.

An analysis of the PSP results and comparison with CFD results was conducted on the baseline case for the MPCV, forward SRB, and aft sections of the SLS-28k configuration at the baseline conditions of $M_{\infty}=0.8, \alpha_{V}=8^{\circ}$, and $\phi_{V}=0^{\circ}$. The PSP and CFD results agreed well with several notable differences. Most of the differences occurred at the location of protuberances such as the aft SRB attachment ring or at areas of high curvature in the geometry such as the SRB nose and the LOX line forward fairing. In general, when a difference between CFD and PSP was observed in the $\triangle C_{P}$ data, the PSP $C_{P}$ measurements had less intensity than that predicted by the CFD (i.e., $C_{P, P S P}$ had a lower absolute value than the predicted $\left.C_{P, C F D}\right)$.

Many of the observations made on the $M_{\infty}=0.8$ baseline case were also observed in the $M_{\infty}=1.3$ data. One exception was the footprint in the $C_{P}$ data from the flow interaction at the SRB nose cone edge, core body, and forward SRB attachment hardware. This flow feature was captured by both CFD and PSP methods but PSP measurements were lower in intensity when compared to the CFD predictions. This flow feature was not present in the $M_{\infty}=1.3$ data. However, the $M_{\infty}=1.3$ data did have a diagonal contour pattern that emanated downstream and outboard from the intersection of each SRB structural ring and the core body. These diagonal contour patterns are evidence of shocks occurring between and over the core and SRBs. The shock patterns were strongest at $\alpha_{V}=8^{\circ}$ and decreased in intensity with decreasing $\alpha_{V}$. The shock patterns became skewed when $\phi_{V}$ was increased and interacted with other shock patterns emanating from the LOX line brackets with the non-zero $\phi_{V}$ condition. CFD and PSP agreed well in measuring the intensity of these shock patterns.

Future areas of study with this existing PSP data set include examining the data for the other two SLS configurations and comparing the $C_{P, P S P}$ with the $C_{P}$ data measured at traditional pressure taps. Another avenue of study is to use other CFD-generated data such as flowfield contours and surface streamlines to explore the various footprints captured by the surface $C_{P}$ data from the PSP and CFD methods. 
Table 1 Model protuberances.

\begin{tabular}{|c|c|}
\hline Vehicle Element & Protuberances \\
\hline \multirow{7}{*}{ Core Stage } & Systems Tunnel $(1)^{*}$ \\
\hline & LOX Feedline and Fairing (2)* \\
\hline & LOX Aft Attach Structure (2)* \\
\hline & Repressurization Lines (1)* \\
\hline & GOX Vent Cover $(1)^{*}$ \\
\hline & Cameras $(8)^{*}$ \\
\hline & Umbilical Attachment Points (4) \\
\hline \multirow{9}{*}{ SRB } & Systems Tunnel (1) \\
\hline & Tunnel Fairings (4) \\
\hline & Rooster Tail (1) \\
\hline & Hold Down Posts (4)* \\
\hline & TVC Brackets (2)* \\
\hline & Kick Ring (1) \\
\hline & Aft Booster Attach Ring (1) \\
\hline & SRB Attach Hardware (Fwd/Aft) \\
\hline & Booster Separation Motors $(8)^{*}$ \\
\hline \multirow{6}{*}{ SLS-10k Upper Stage } & LAS Tower (1) \\
\hline & LAS Nozzles (4)* \\
\hline & LAS Systems Tunnel (1)* \\
\hline & MPCV Umbilical (1)* \\
\hline & Upper Stage Systems Tunnel (1)* \\
\hline & Cameras $(1)^{*}$ \\
\hline \multirow{2}{*}{ SLS-27k Upper Stage } & Upper Stage Systems Tunnel (1)* \\
\hline & Cameras $(2)^{*}$ \\
\hline \multirow{6}{*}{ SLS-28k Upper Stage } & LAS Tower (1) \\
\hline & LAS Nozzles (4)* \\
\hline & LAS Systems Tunnel (1)* \\
\hline & MPCV Umbilical (1)* \\
\hline & Upper Stage Systems Tunnel (1)* \\
\hline & Cameras $(3)^{*}$ \\
\hline
\end{tabular}

* Removable protuberance for clean configuration testing 
Table 2 Flow and model conditions in wind-tunnel test.

\begin{tabular}{|c|c|}
\hline $\begin{array}{c}\text { Flow and Model } \\
\text { Parameter }\end{array}$ & Dimension \\
\hline \multicolumn{2}{|c|}{ UPWT 11- by 11-Foot Transonic Wind Tunnel } \\
\hline$M_{\infty}$ & $0.3,0.5,0.8,0.9,0.95,1.05,1.1,1.2,1.3,1.4$ \\
\hline$R e$ & $3 \times 10^{6} / \mathrm{ft}, 5 \times 10^{6} / \mathrm{ft}$ \\
\hline$\alpha_{V}$ & $-8^{\circ}$ to $8^{\circ}$ in $1^{\circ}$ increments \\
\hline$\beta_{V}$ & $-^{\circ}$ to $8^{\circ}$ in $4^{\circ}$ increments \\
\hline$\phi_{V}$ & $0^{\circ}$ to $180^{\circ}$ in $22.5^{\circ}$ increments, $270^{\circ}$ \\
\hline \multicolumn{2}{|c|}{ UPWT 9- by 7-Foot Supersonic Wind Tunnel } \\
\hline$M_{\infty}$ & $1.55,1.6,1.8,2.0,2.5$ \\
\hline$R e$ & $3 \times 10^{6} / \mathrm{ft}, 5 \times 10^{6} / \mathrm{ft}$ \\
\hline$\alpha_{V}$ & $-8^{\circ}$ to $8^{\circ}$ in $1^{\circ}$ increments \\
\hline$\beta_{V}$ & $-8^{\circ}$ to $8^{\circ}$ in $1^{\circ}$ increments \\
\hline$\phi_{V}$ & $0^{\circ}, 90^{\circ}, 180^{\circ}$ \\
\hline
\end{tabular}

Table 3 Trip dot sizes and locations used in wind-tunnel test.

\begin{tabular}{|c|c|}
\hline Location & Trip Dot Height [in] \\
\hline UPWT 11- by 11-Foot Transonic Wind Tunnel \\
\hline LAS Tower & 0.0056 \\
\hline LAS/Orion Fairing & 0.0080 \\
\hline SRB Nose Cones & 0.0060 \\
\hline UPWT 9- by 7-Foot Supersonic Wind Tunnel \\
\hline LAS Tower & 0.0075 \\
\hline LAS/Orion Fairing & 0.0114 \\
\hline SRB Nose Cones & 0.0086 \\
\hline
\end{tabular}


Table 4 Surface grid elements.

\begin{tabular}{|c|c|}
\hline Grid Elements & Measurement \\
\hline \multicolumn{2}{|c|}{ Wind-tunnel Surface Grid } \\
\hline Patches & 2,233 \\
\hline Nodes & 264,944 \\
\hline Cells & 224,677 \\
\hline \multicolumn{2}{|c|}{ CFD Surface Grid } \\
\hline Patches & 417 \\
\hline Nodes & 761,403 \\
\hline Cells & $1,446,234$ \\
\hline
\end{tabular}

Table 5 Processing PSP image-intensity data to PSP surface-pressure data.

\begin{tabular}{|c|l|}
\hline Step & Description \\
\hline 1 & Generate a surface grid for the wind tunnel geometry \\
\hline 2 & Map and average camera pixels onto surface grid nodes \\
\hline 3 & Apply a pretest calibration to the image intensity data \\
\hline 4 & Apply a prerun 'pump down' calibration to step 3 data \\
\hline 5 & $\begin{array}{l}\text { Apply a pressure tap calibration global offset to step } 4 \\
\text { data }\end{array}$ \\
\hline
\end{tabular}

Table 6 PSP and CFD $C_{P}$ comparison process.

\begin{tabular}{|c|l|}
\hline Step & Description \\
\hline 1 & Find no-data patches on PSP surface grid \\
\hline 2 & Find anomalous patches on PSP surface grid \\
\hline 3 & Combine patches in CFD surface grid \\
\hline 4 & Interpolate CFD solution onto PSP surface grid \\
\hline 5 & $\begin{array}{l}\text { Calculate the delta between the CFD and PSP surface } \\
\text { pressures on the PSP surface grid }\end{array}$ \\
\hline 6 & $\begin{array}{l}\text { Generate contour plots of the delta surface pressures } \\
\text { for analysis }\end{array}$ \\
\hline
\end{tabular}

Table 7 Subset of run $\log$ for present investigation on the SLS-28k configuration.

\begin{tabular}{|c|c|c|c|c|c|c|}
\hline$R e$ & \multicolumn{6}{|c|}{$3 \times 10^{6} / \mathrm{ft}$} \\
\hline$\beta_{V}$ & \multicolumn{5}{|c|}{$0^{\circ}$} \\
\hline$M_{\infty}$ & \multicolumn{3}{|c|}{0.8} & \multicolumn{3}{c|}{1.3} \\
\hline \multirow{2}{*}{$\alpha_{V}$} & \multicolumn{3}{|c|}{$\phi_{V}$} & \multicolumn{3}{c|}{$\phi_{V}$} \\
\cline { 2 - 8 } & $0^{\circ}$ & $45^{\circ}$ & $90^{\circ}$ & $0^{\circ}$ & $45^{\circ}$ & $90^{\circ}$ \\
\hline $0^{\circ}$ & $\sqrt{ }$ & & & $\sqrt{ }$ & & \\
\hline $2^{\circ}$ & $\sqrt{ }$ & $\sqrt{ }$ & $\sqrt{ }$ & $\sqrt{ }$ & $\sqrt{ }$ & $\sqrt{ }$ \\
\hline $4^{\circ}$ & $\sqrt{ }$ & $\sqrt{ }$ & $\sqrt{ }$ & $\sqrt{ }$ & $\sqrt{ }$ & $\sqrt{ }$ \\
\hline $8^{\circ}$ & $\sqrt{ }$ & $\sqrt{ }$ & $\sqrt{ }$ & $\sqrt{ }$ & $\sqrt{ }$ & $\sqrt{ }$ \\
\hline
\end{tabular}




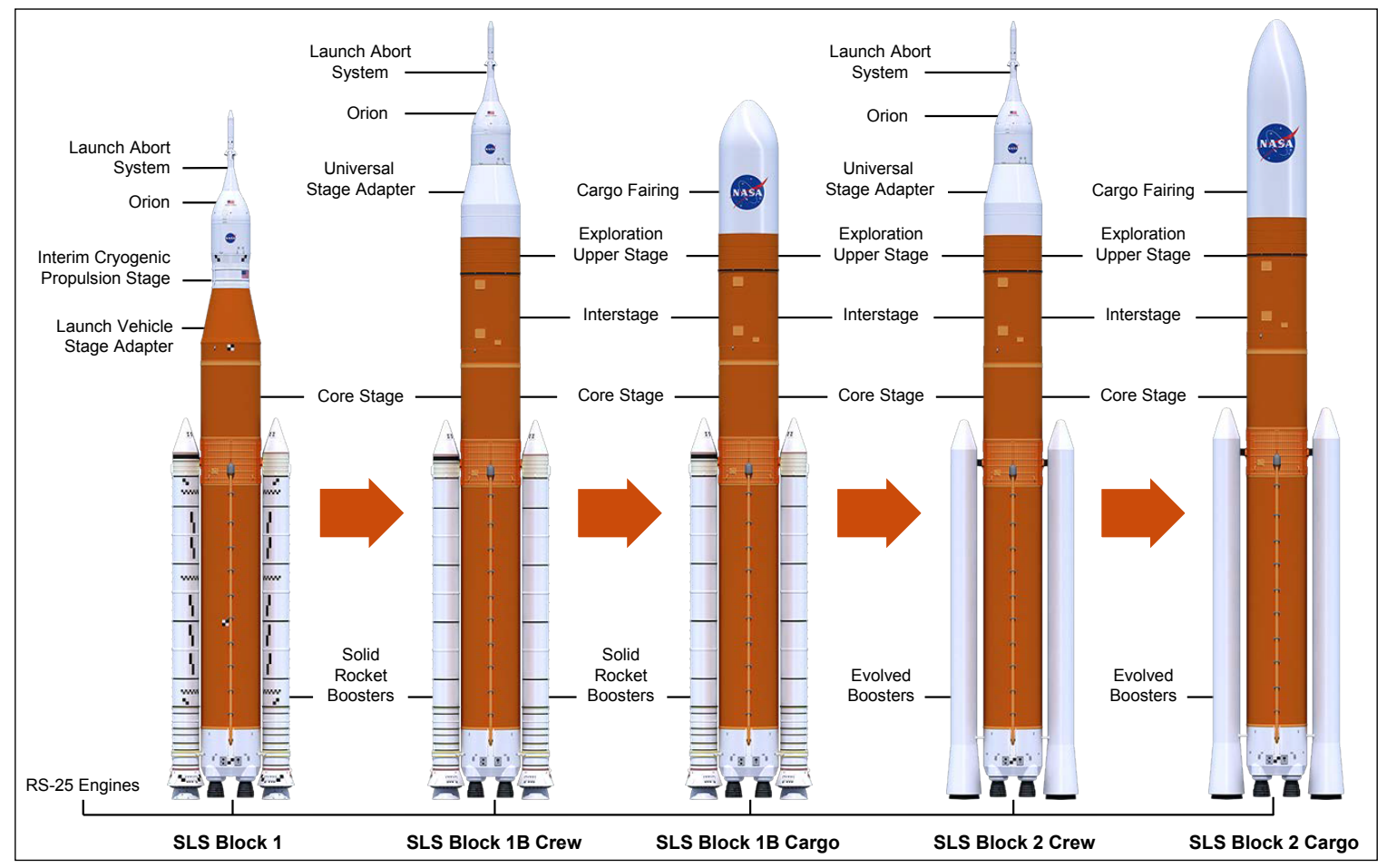

Fig. 1 Artist renderings of the SLS vehicle configurations [9].

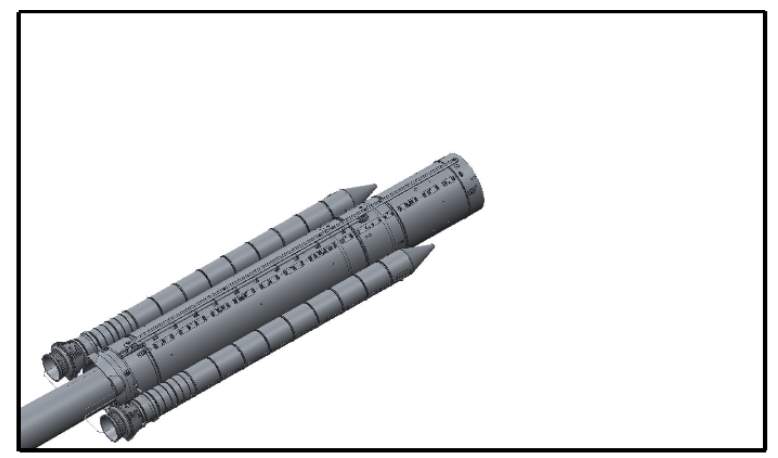

(a) Common stack.

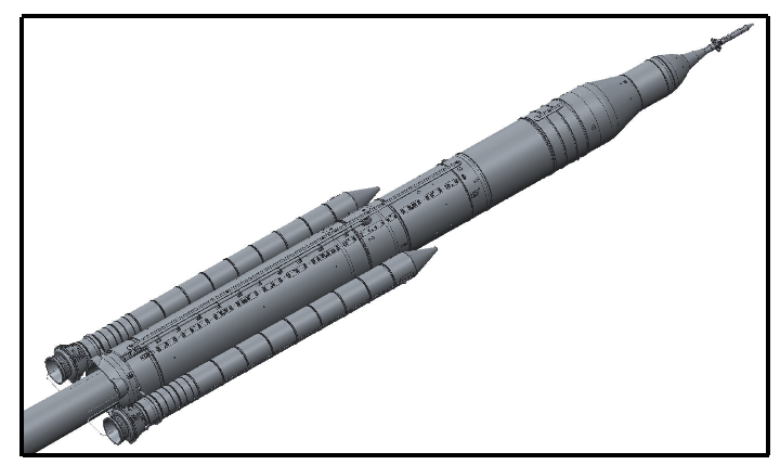

(c) SLS-28k configuration.

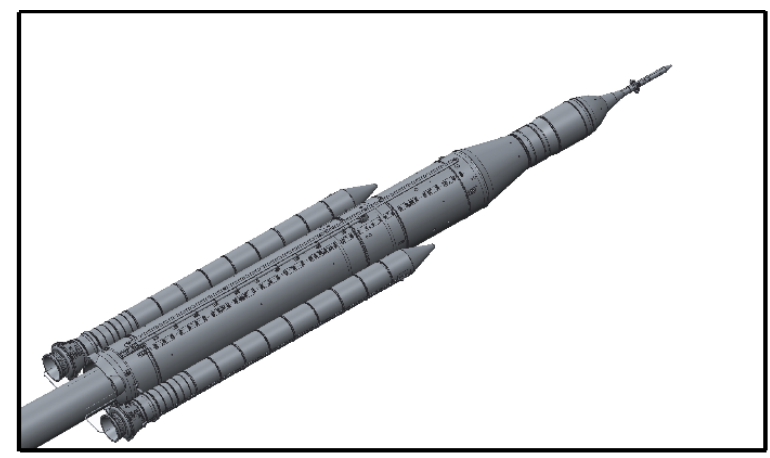

(b) SLS-10k configuration.

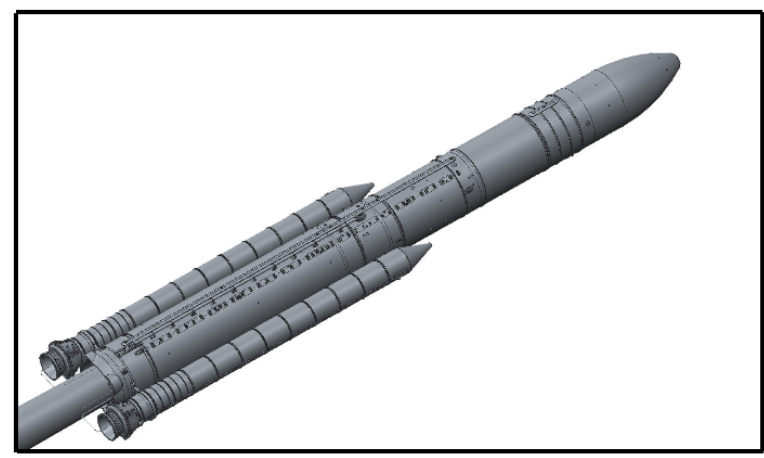

(d) SLS-27k configuration.

Fig. 2 CAD models of assembled wind-tunnel configurations. 


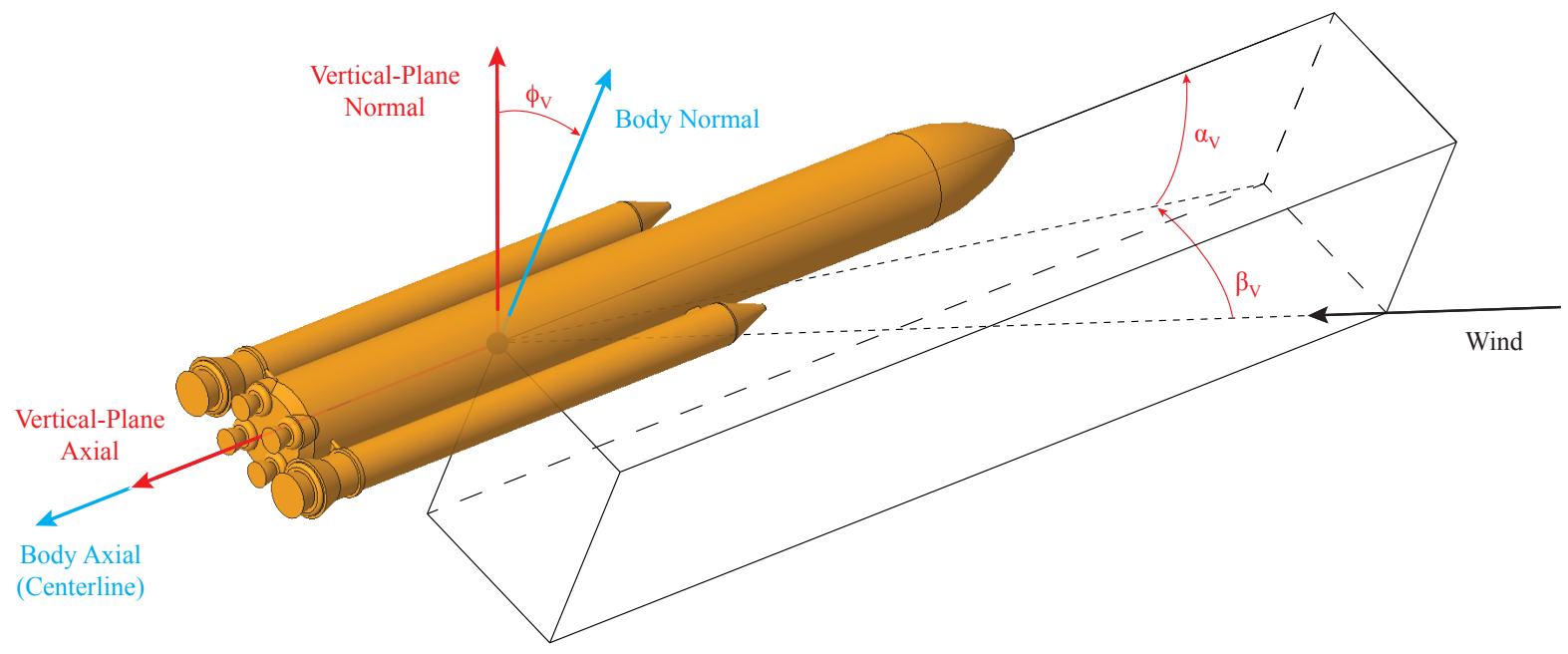

Fig. 3 Sketch of vertical plane axis system.

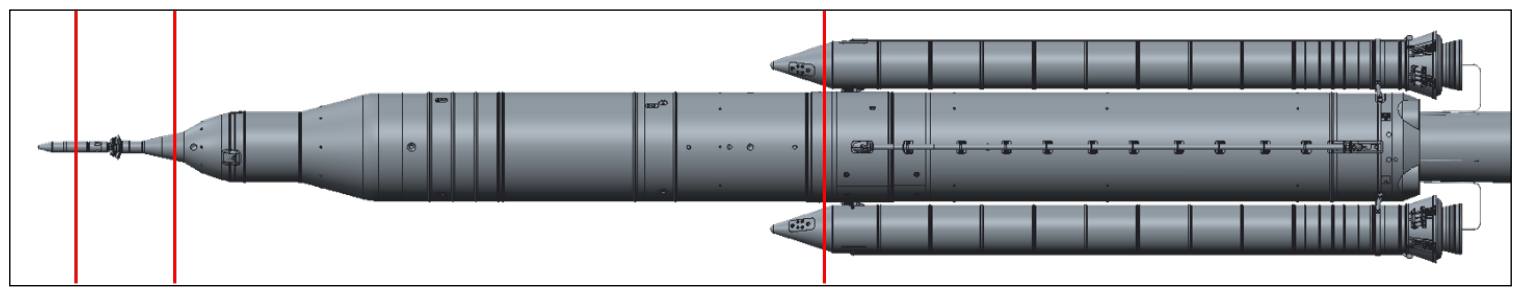

Fig. 4 Axial locations of the boundary layer trip dots for the SLS-28k configuration.

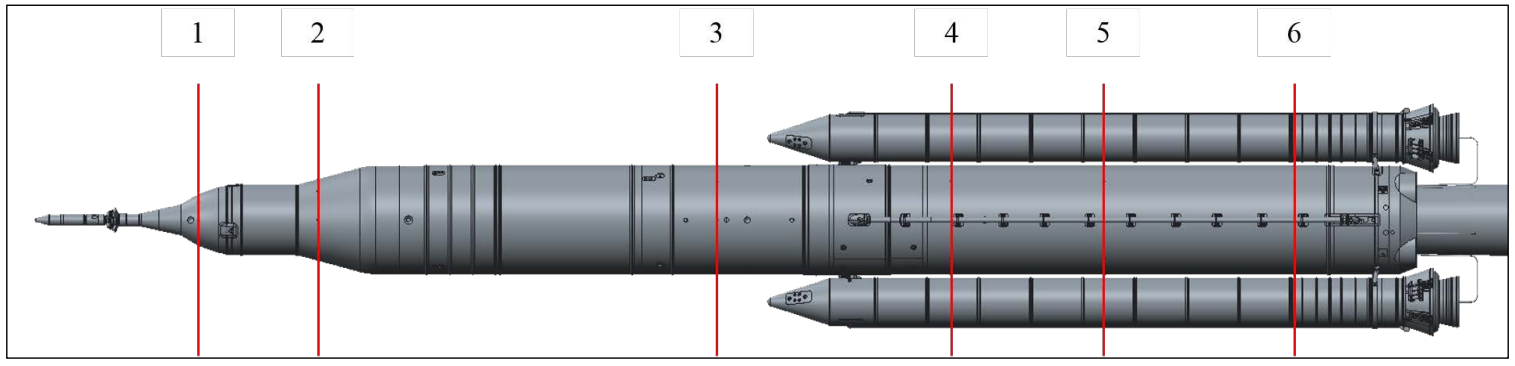

Fig. 5 Axial locations of the surface pressure taps for the SLS-28k configuration. 


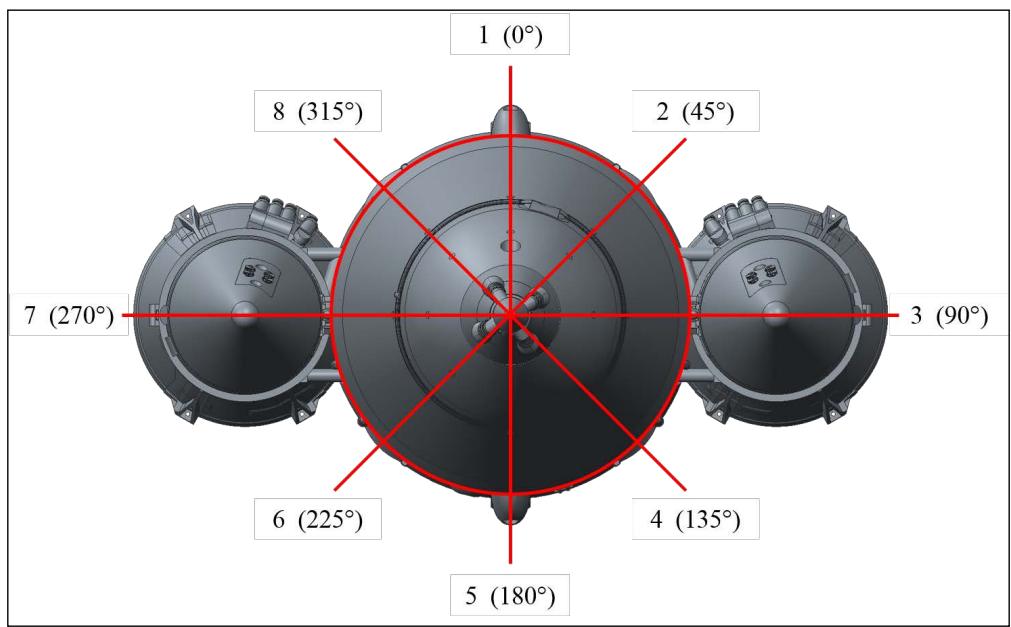

Fig. 6 Radial locations of the surface pressure taps on the core component of the SLS-28k configuration.

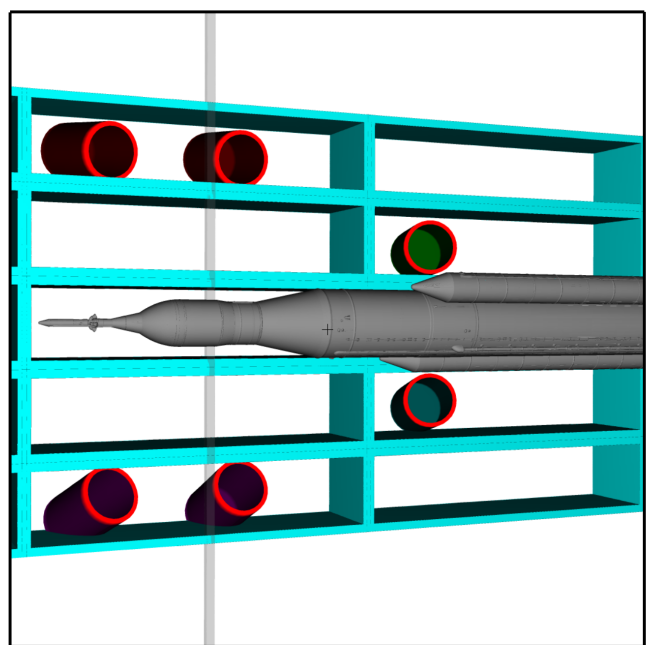

(a) Front port side view

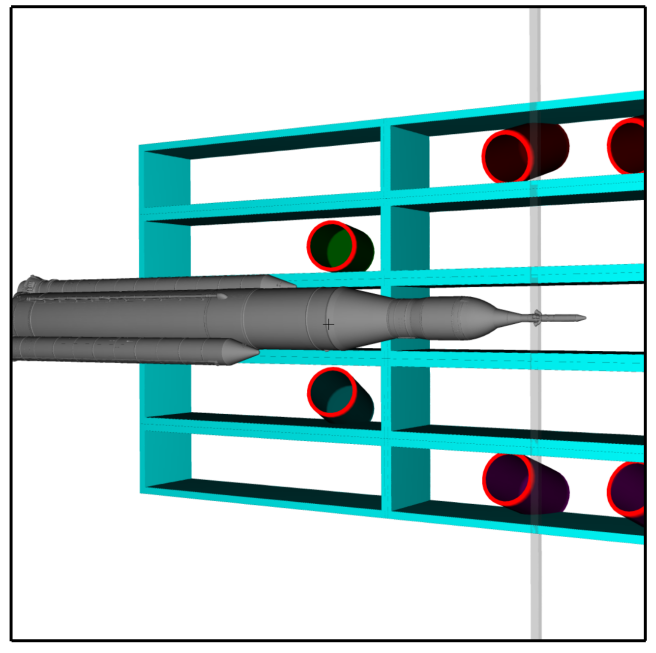

(c) Front starboard side view.

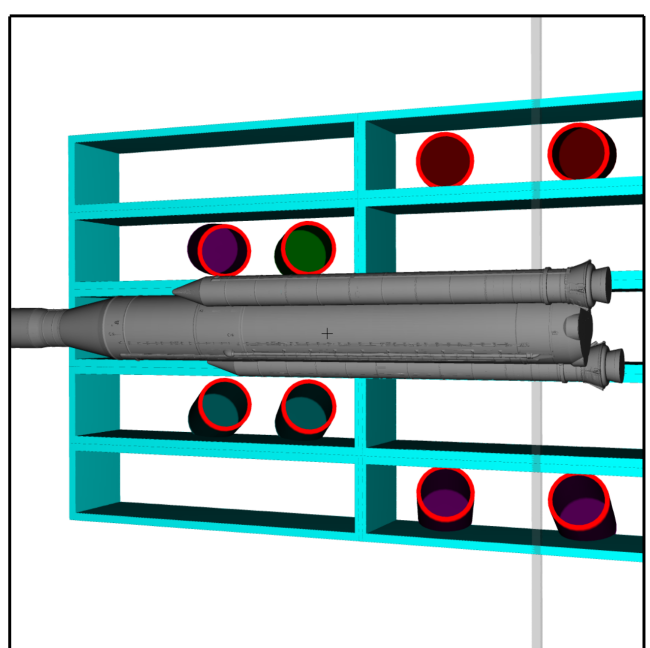

(b) Aft port side view.

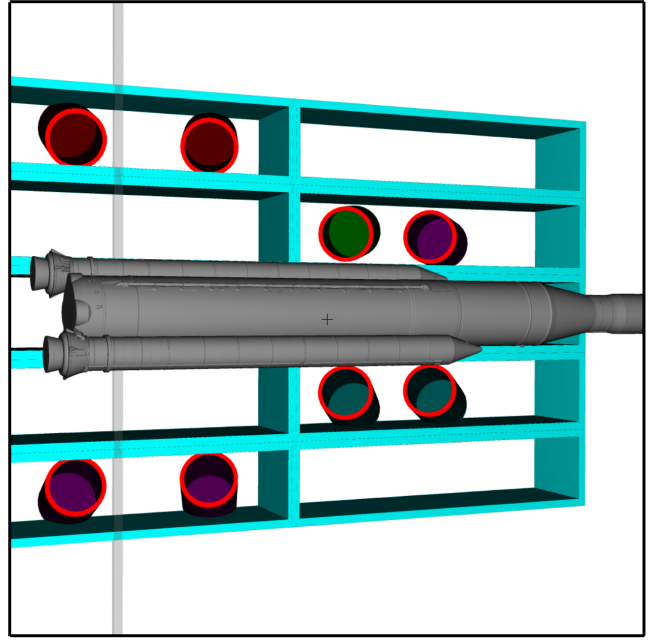

(d) Aft starboard side view.

Fig. 7 Various views of the lamps and cameras set up in the slotted side windows of the test section for the SLS-28k configuration at $\alpha_{V}=0^{\circ}$ and $\phi_{V}=4^{\circ}$. 


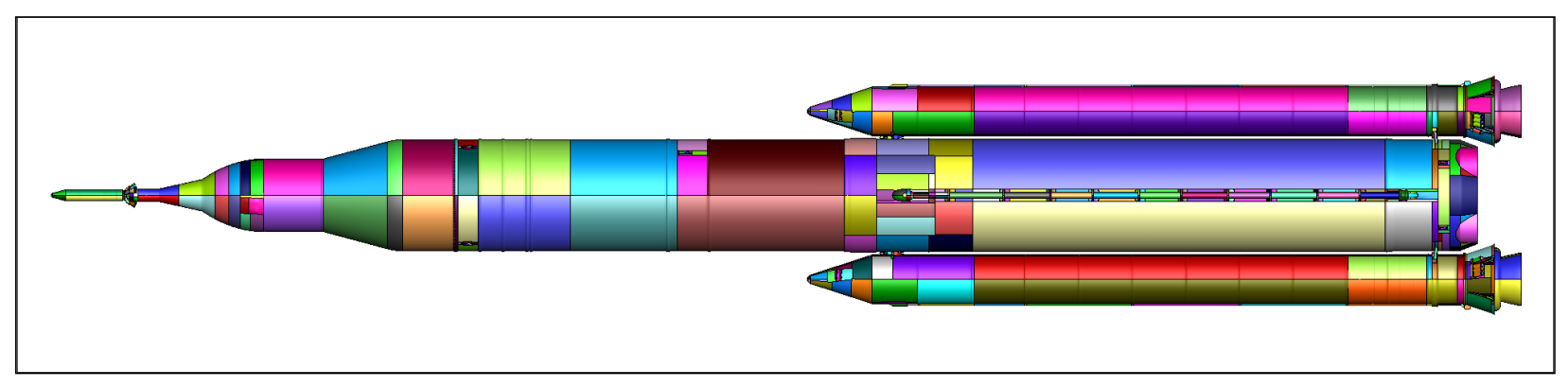

Fig. 8 Sketch of patches on the upper surface of the wind-tunnel surface grid for the SLS-28k configuration.

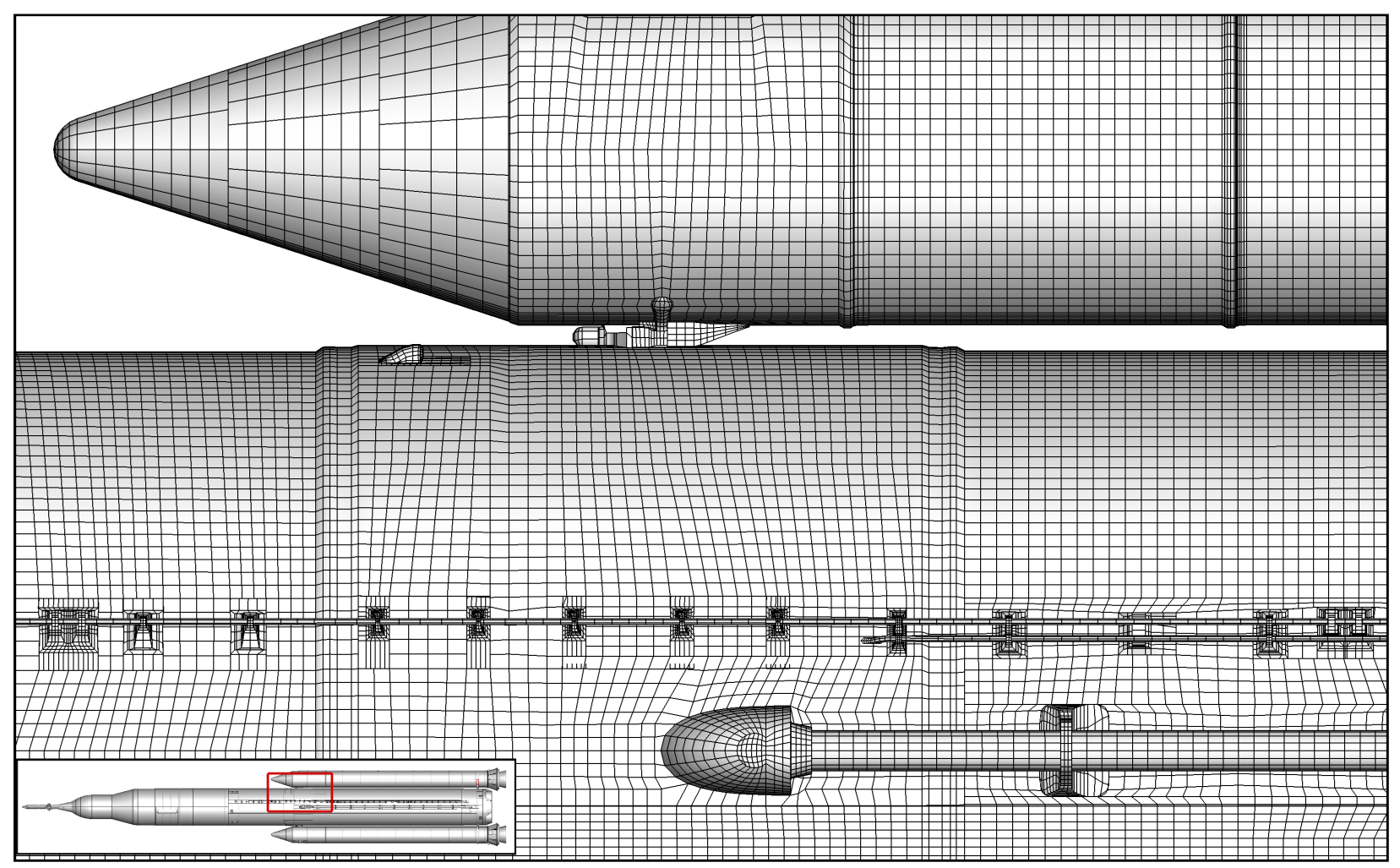

Fig. 9 Lower SLS-28k wind-tunnel surface grid at the forward SRB attachment location. 


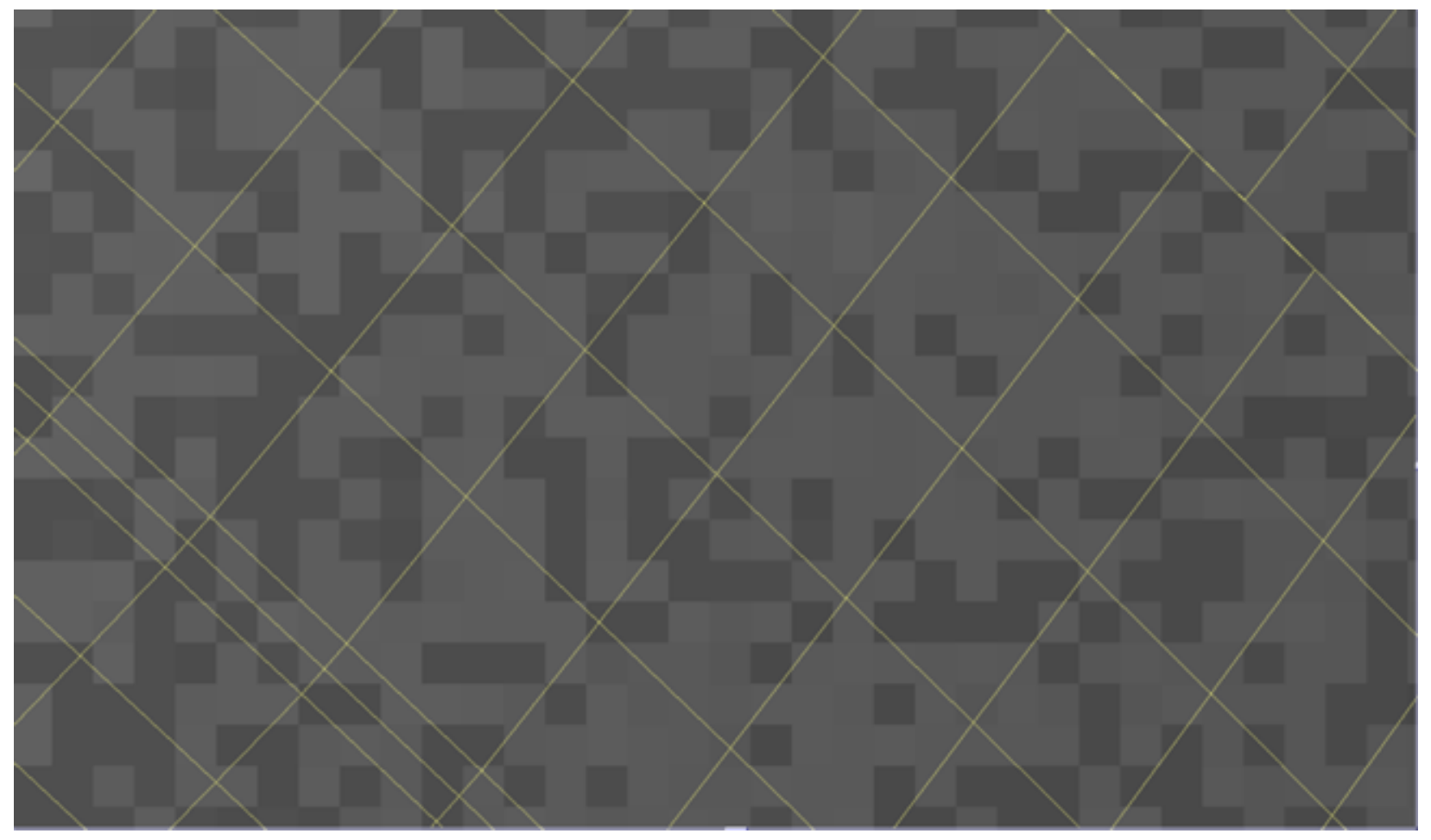

Fig. 10 Camera pixels and PSP surface grid cells.

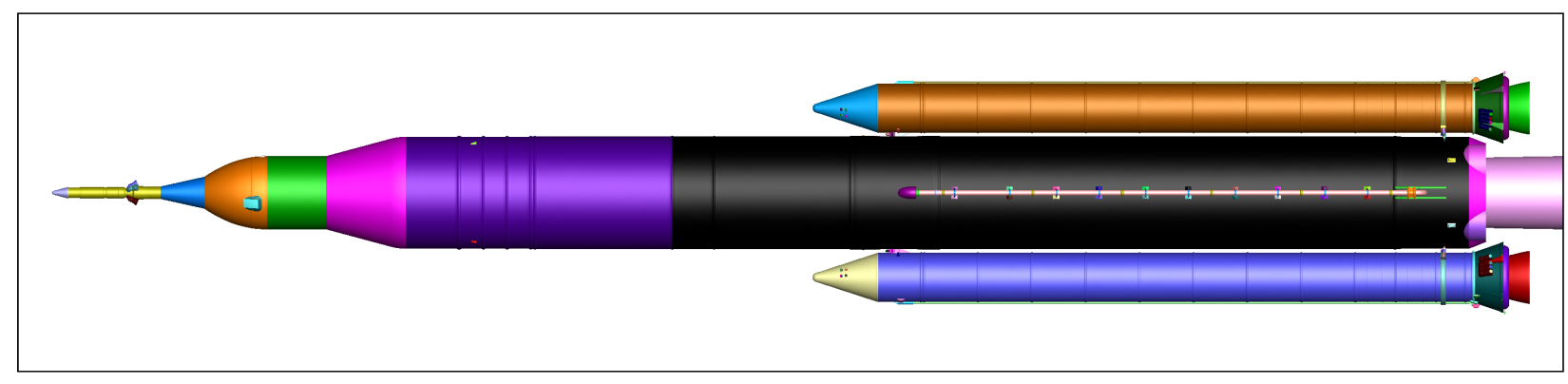

Fig. 11 Sketch of patches on the upper surface of the CFD surface grid of the SLS-28k configuration. 


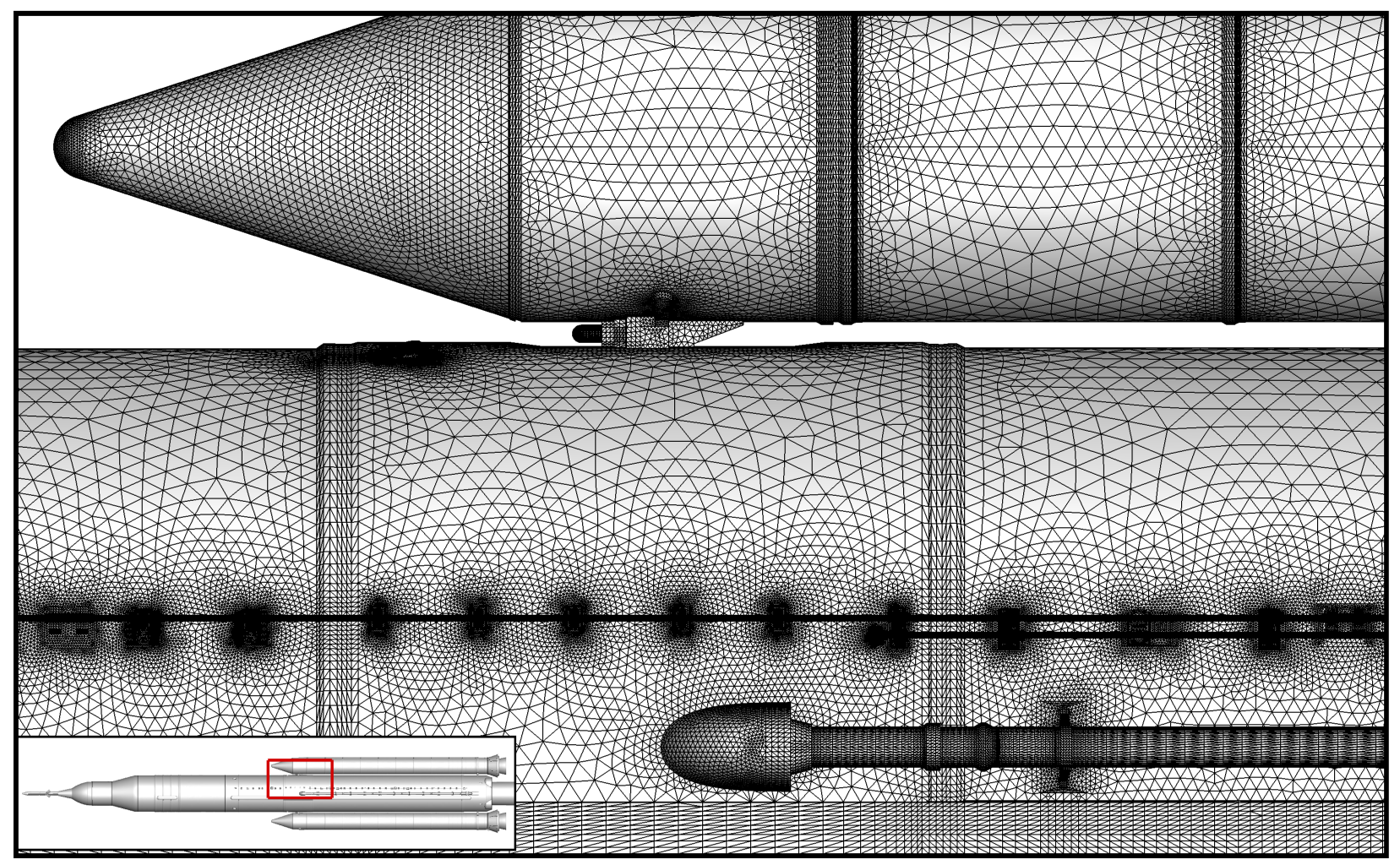

Fig. 12 Lower SLS-28k CFD surface grid at the forward SRB attachment location.

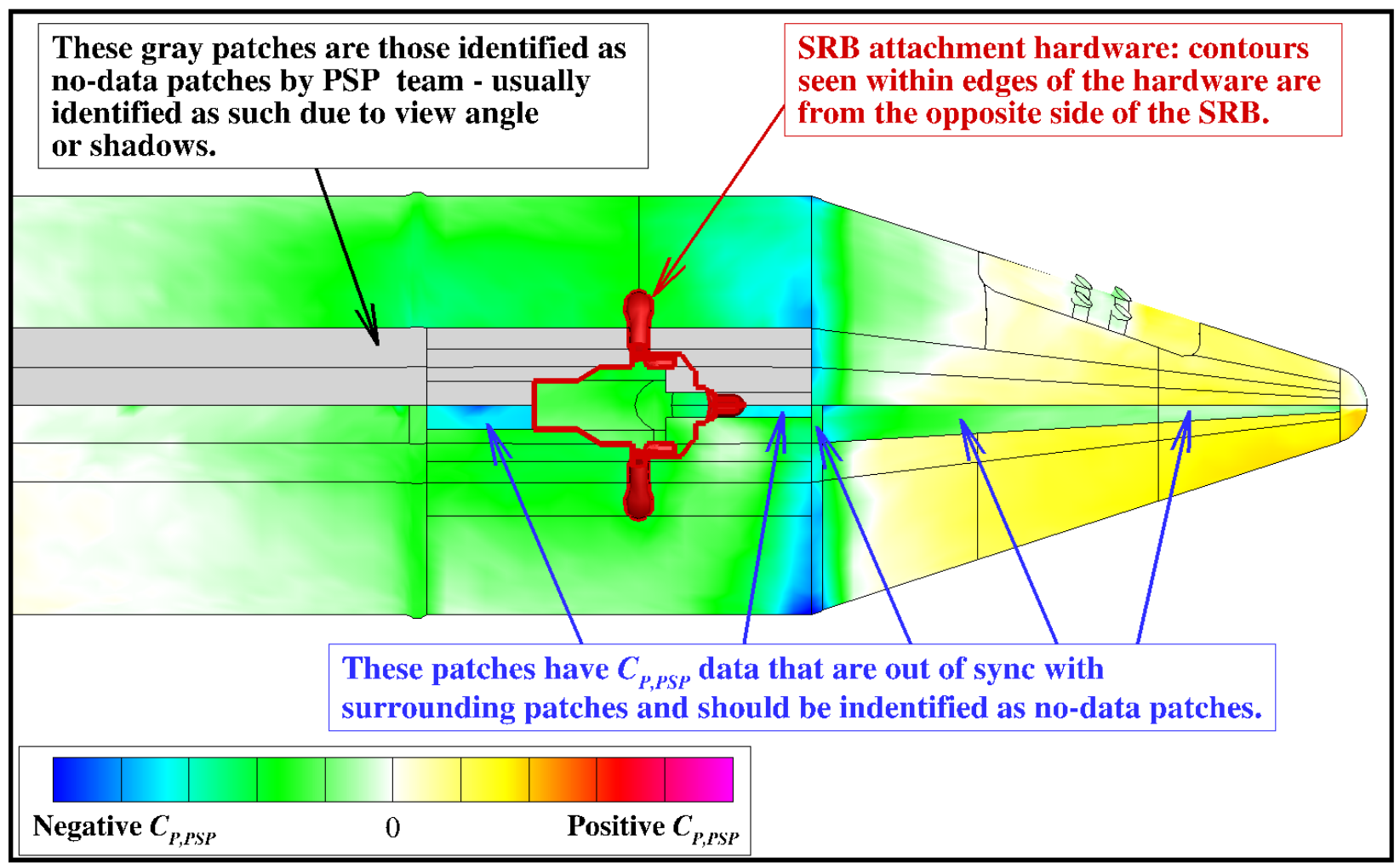

Fig. $13 C_{P, P S P}$ data on the inboard side of the left SRB at $M_{\infty}=0.8, \alpha_{V}=4^{\circ}$, and $\phi_{V}=0^{\circ}$. 


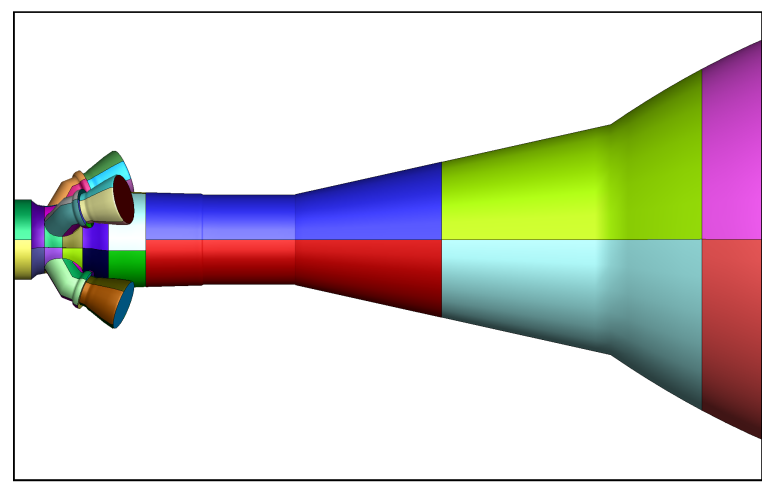

(a) Patches on PSP surface grid.

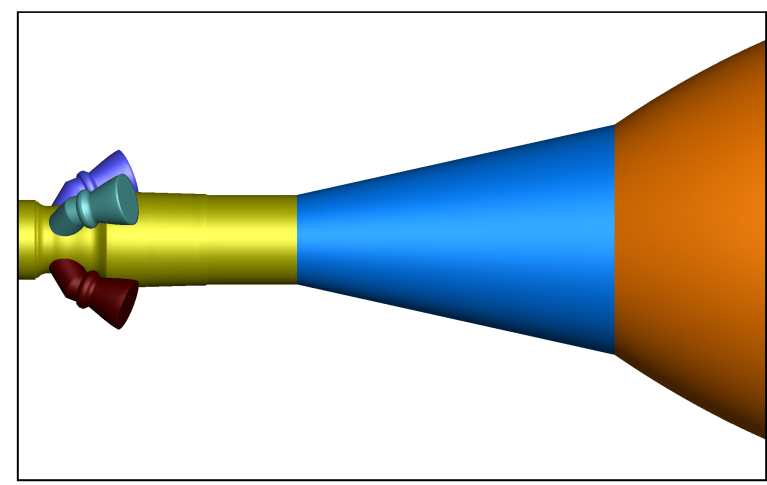

(b) Patches on CFD surface grid.

Fig. 14 Sketches of patches on the upper surface of the PSP and CFD surface grids on a portion of the LAS/MPCV components of the SLS-28k configuration.

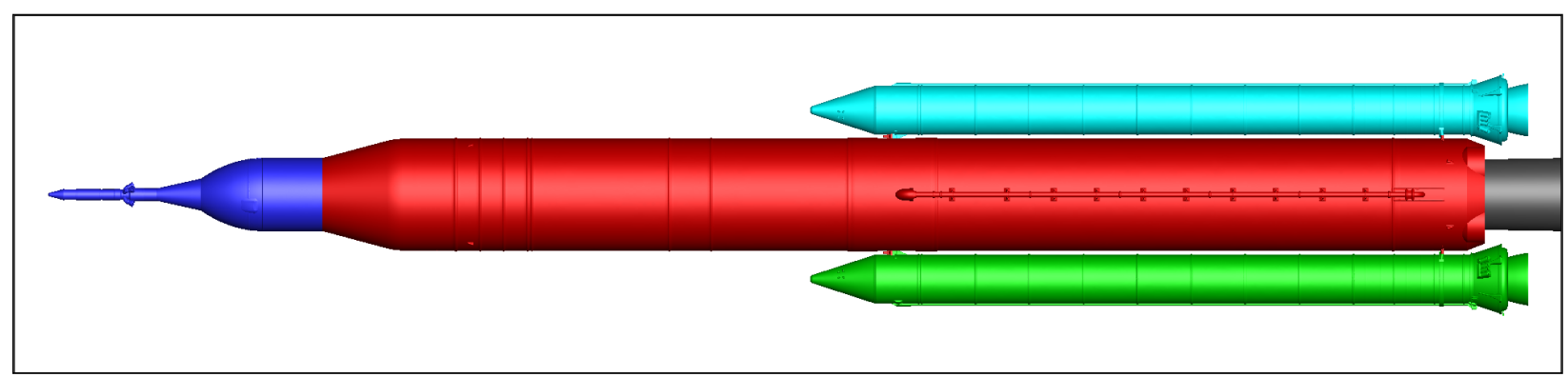

Fig. 15 Sketch of patches on the upper surface of the SLS-28k CFD surface grid.

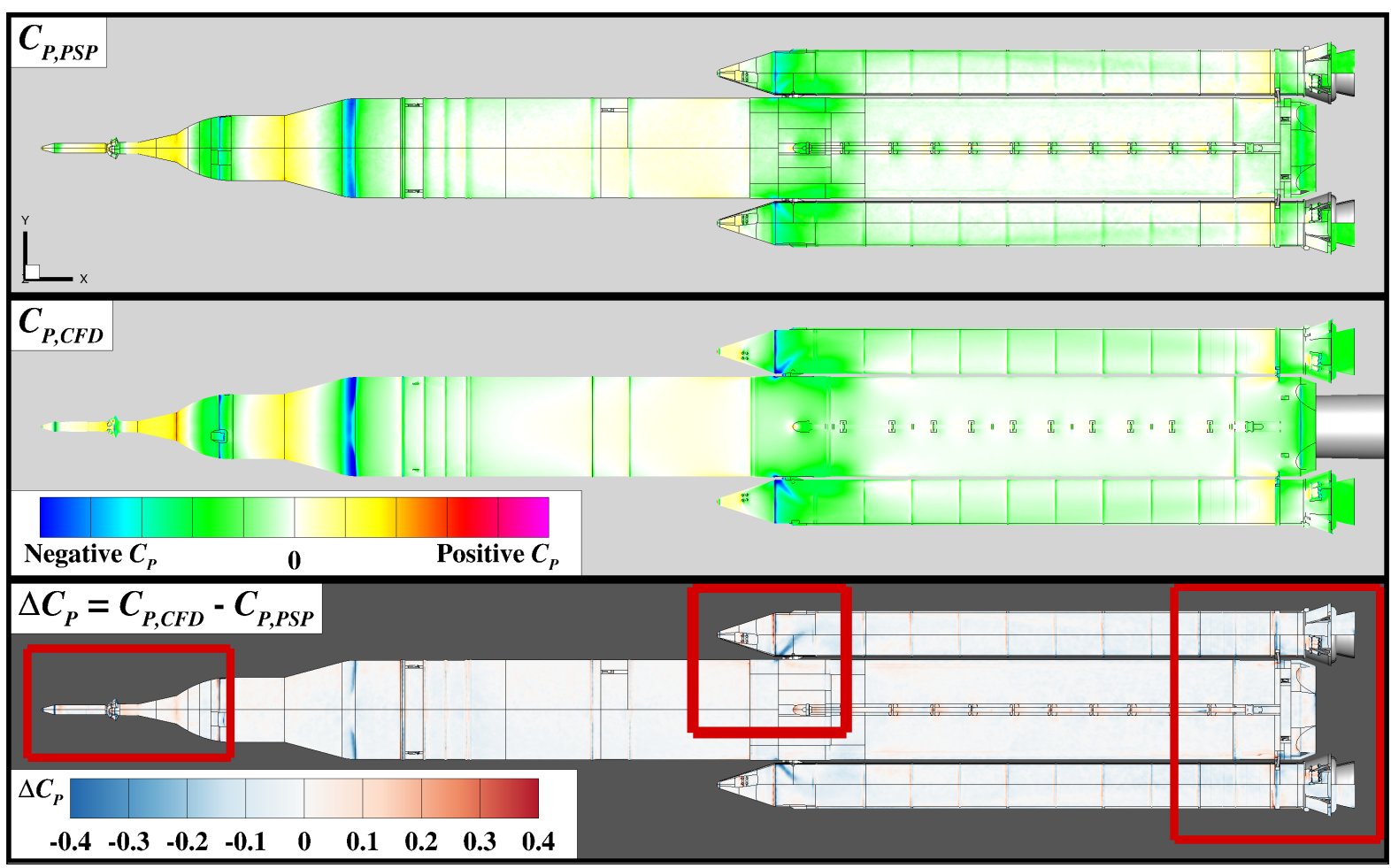

(a) Upper surface.

Fig. 16 Comparison of PSP and CFD surface pressure coefficient data on the SLS-28k configuration at $M_{\infty}=0.8, \alpha_{V}=8^{\circ}$, and $\phi_{V}=0^{\circ}$. 


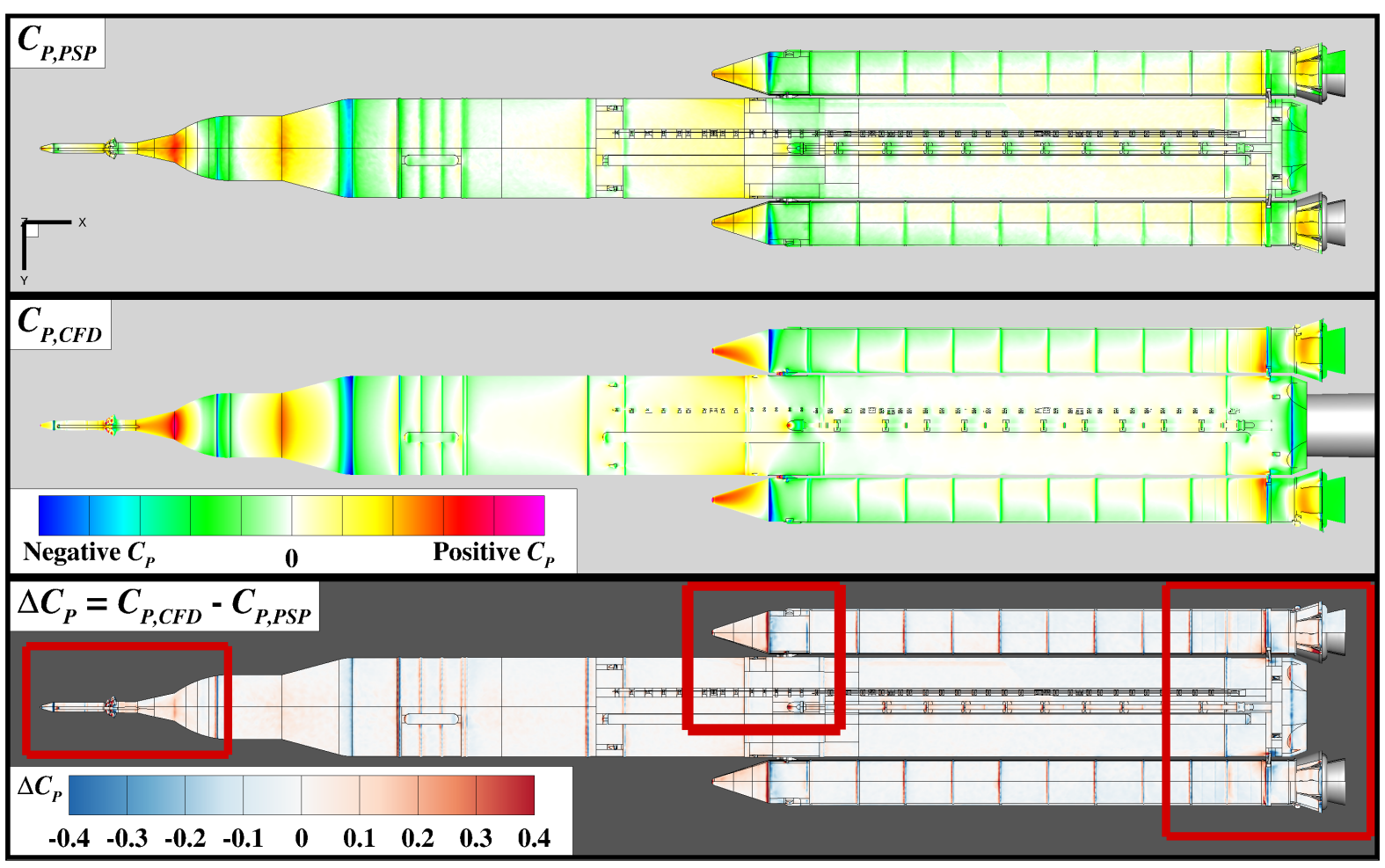

(b) Lower surface.

Fig. 16 Concluded.

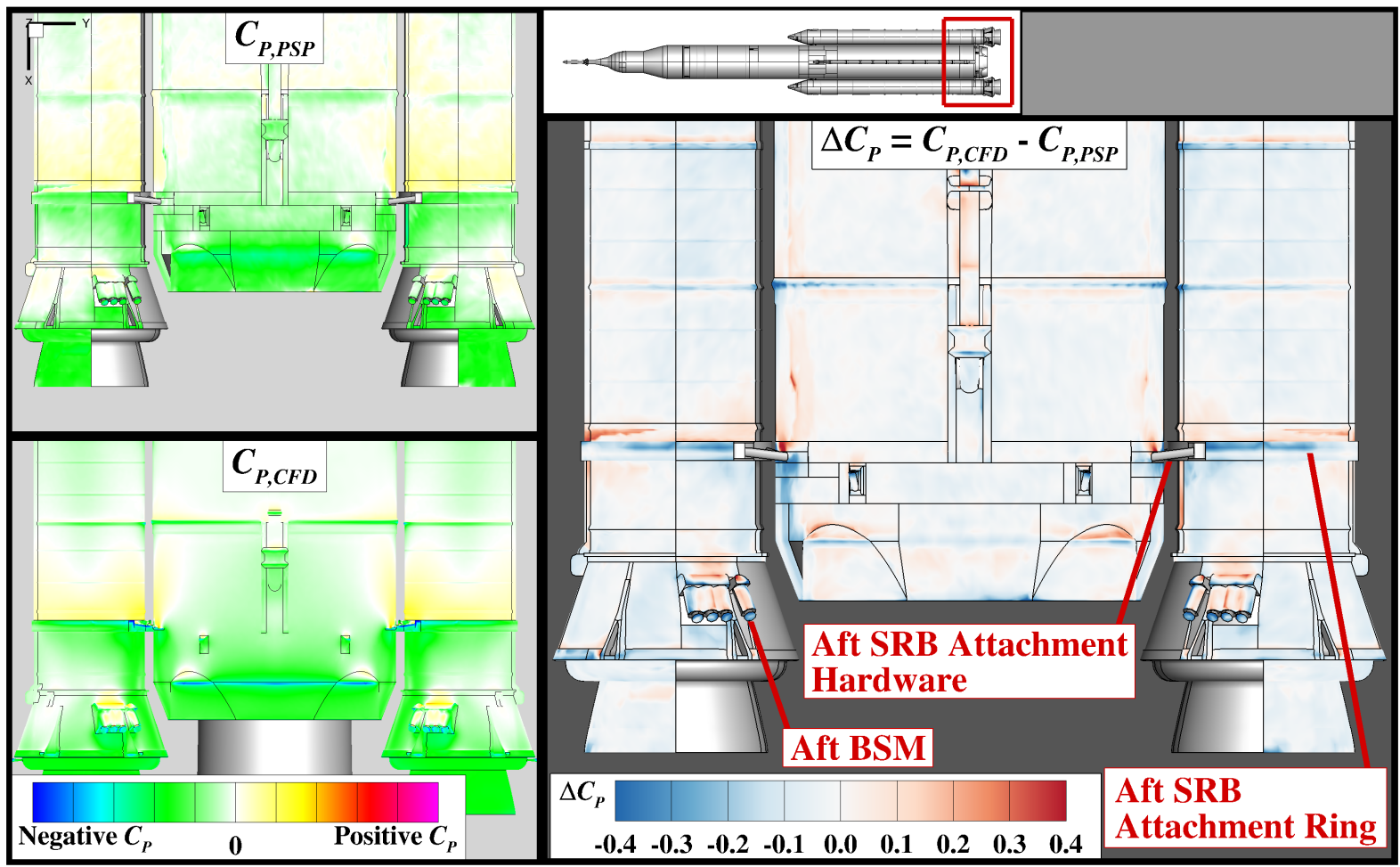

(a) Upper surface.

Fig. 17 Comparison of PSP and CFD surface pressure coefficient data on the aft section of the SLS-28k configuration at $M_{\infty}=0.8, \alpha_{V}=8^{\circ}$, and $\phi_{V}=0^{\circ}$. 


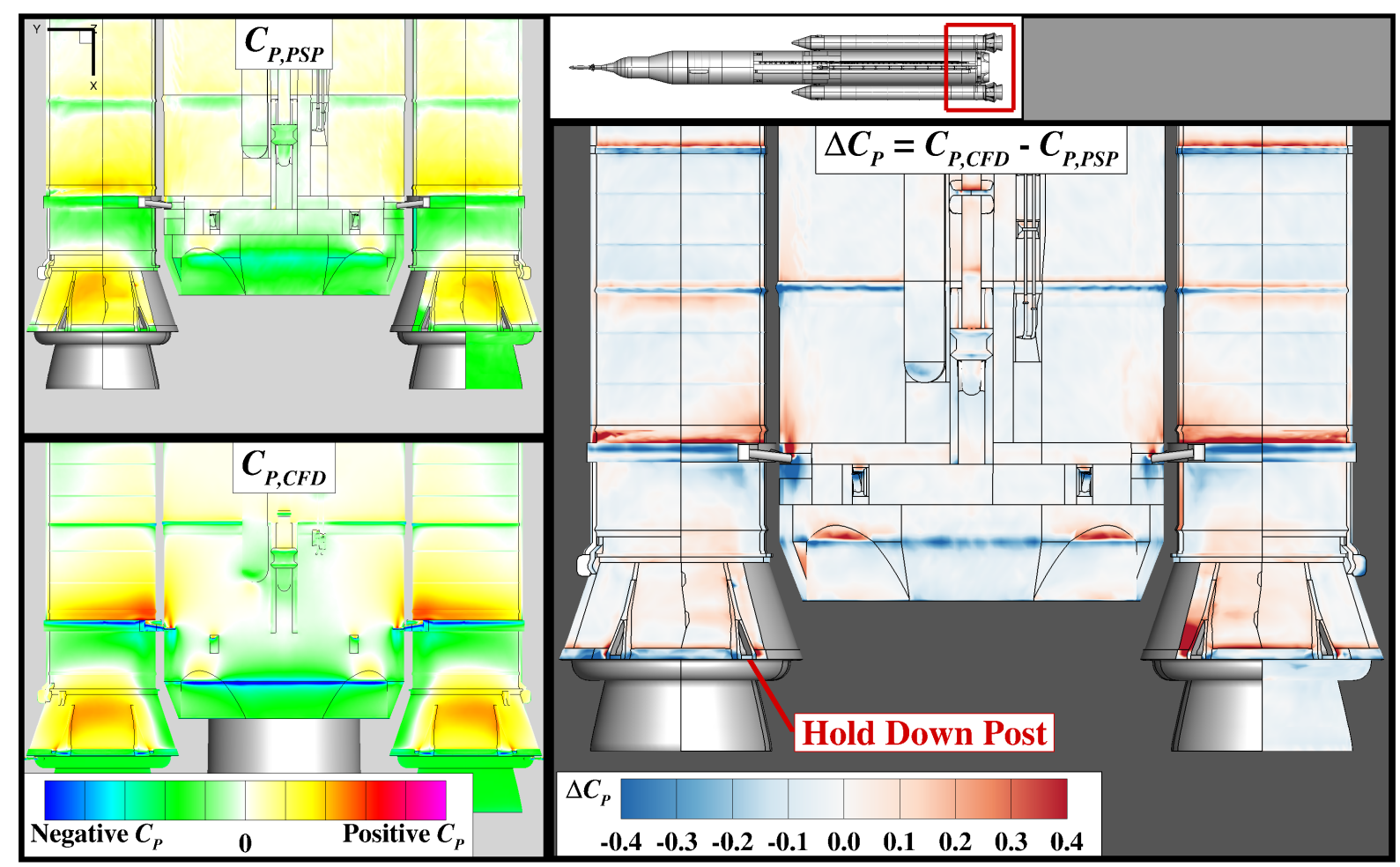

(b) Lower surface.

Fig. 17 Concluded.

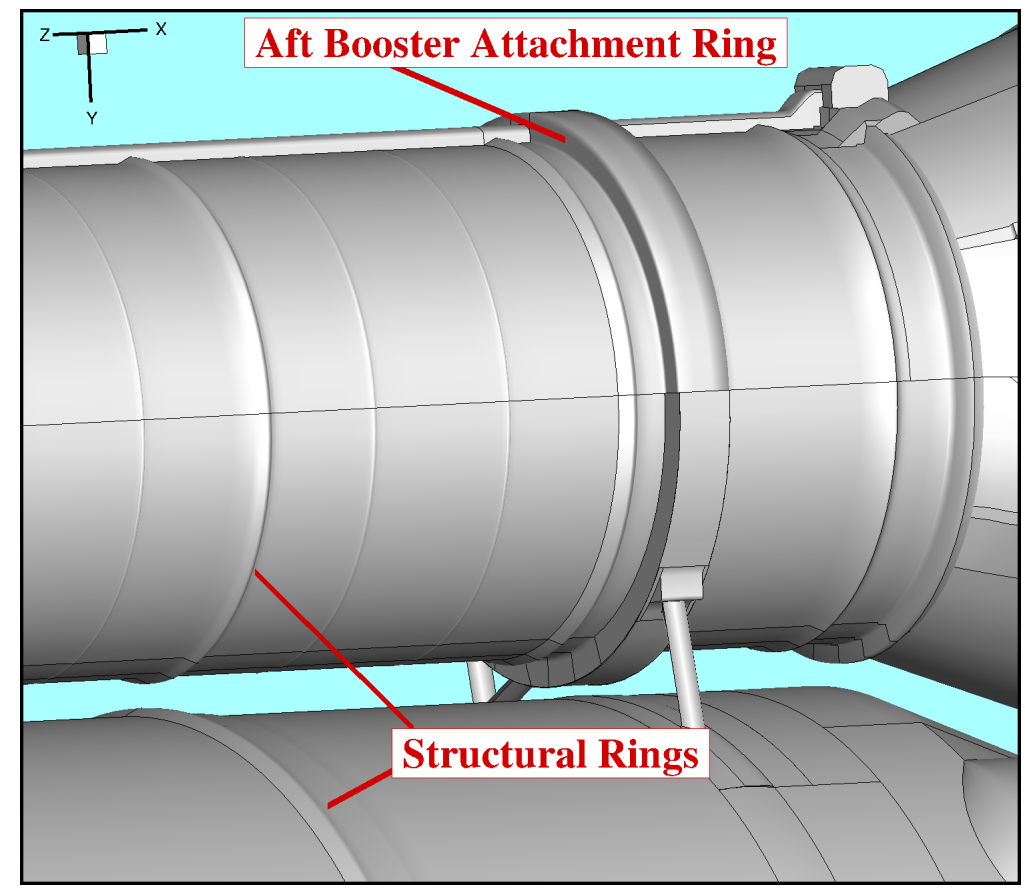

Fig. 18 View of structural rings and port-side aft booster attachment ring on the lower surface. 


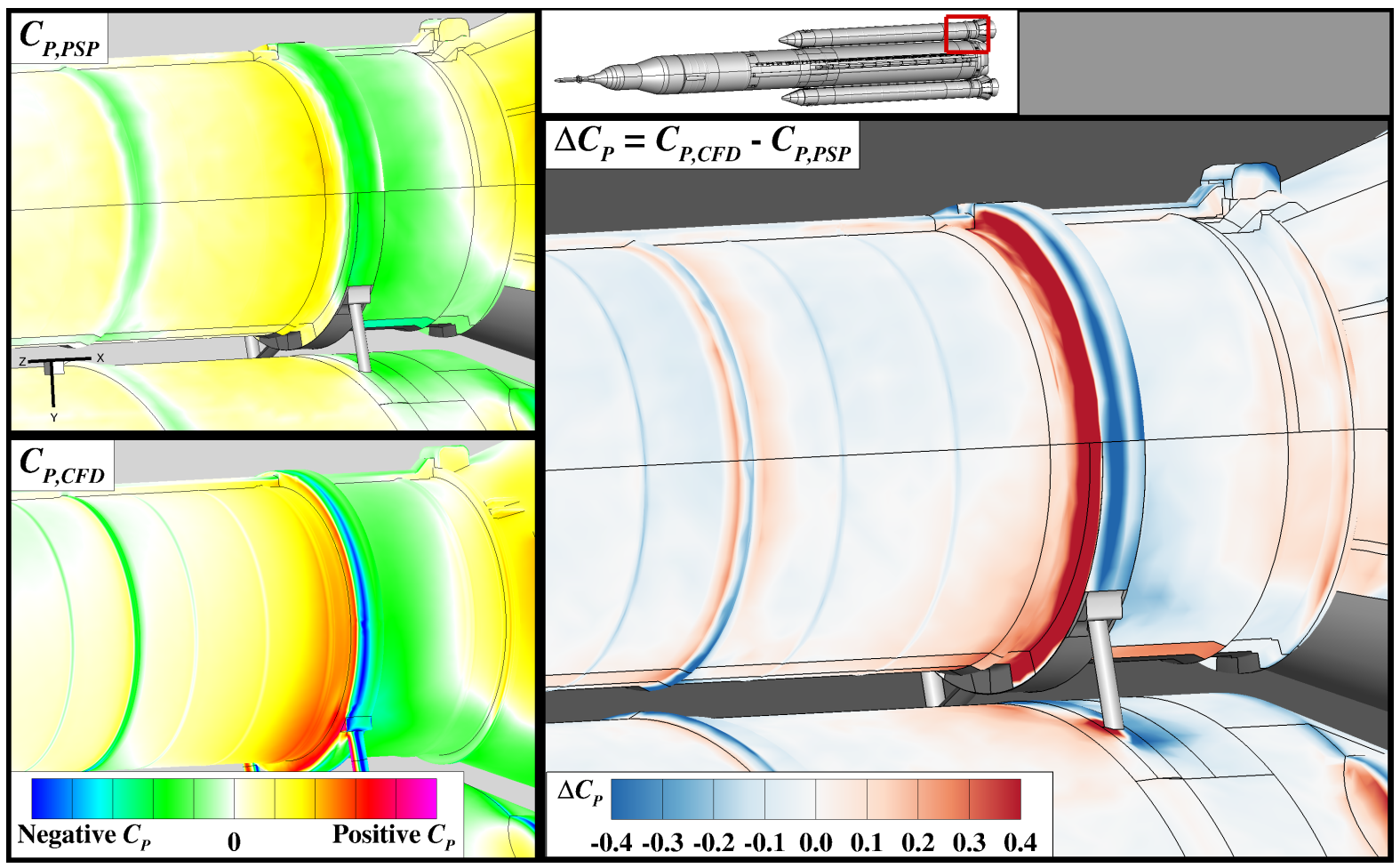

Fig. 19 Comparison of PSP and CFD surface pressure coefficient data near the port-side aft booster attachment ring on the lower surface of the SLS-28k configuration at $M_{\infty}=0.8, \alpha_{V}=8^{\circ}$, and $\phi_{V}=0^{\circ}$.

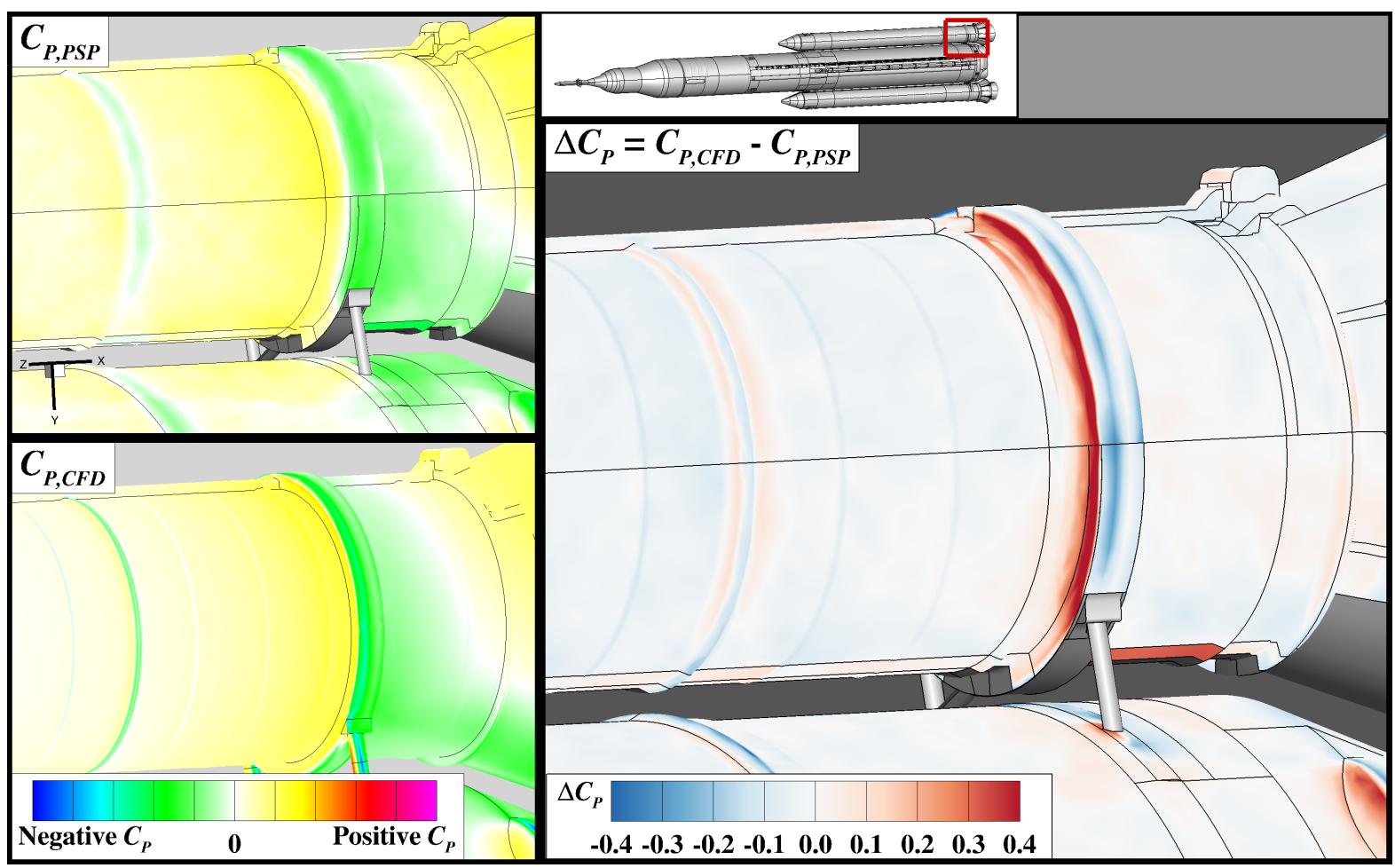

Fig. 20 Comparison of PSP and CFD surface pressure coefficient data near the port-side aft booster attachment ring on the lower surface of the SLS-28k configuration at $M_{\infty}=0.8, \alpha_{V}=0^{\circ}$, and $\phi_{V}=0^{\circ}$. 


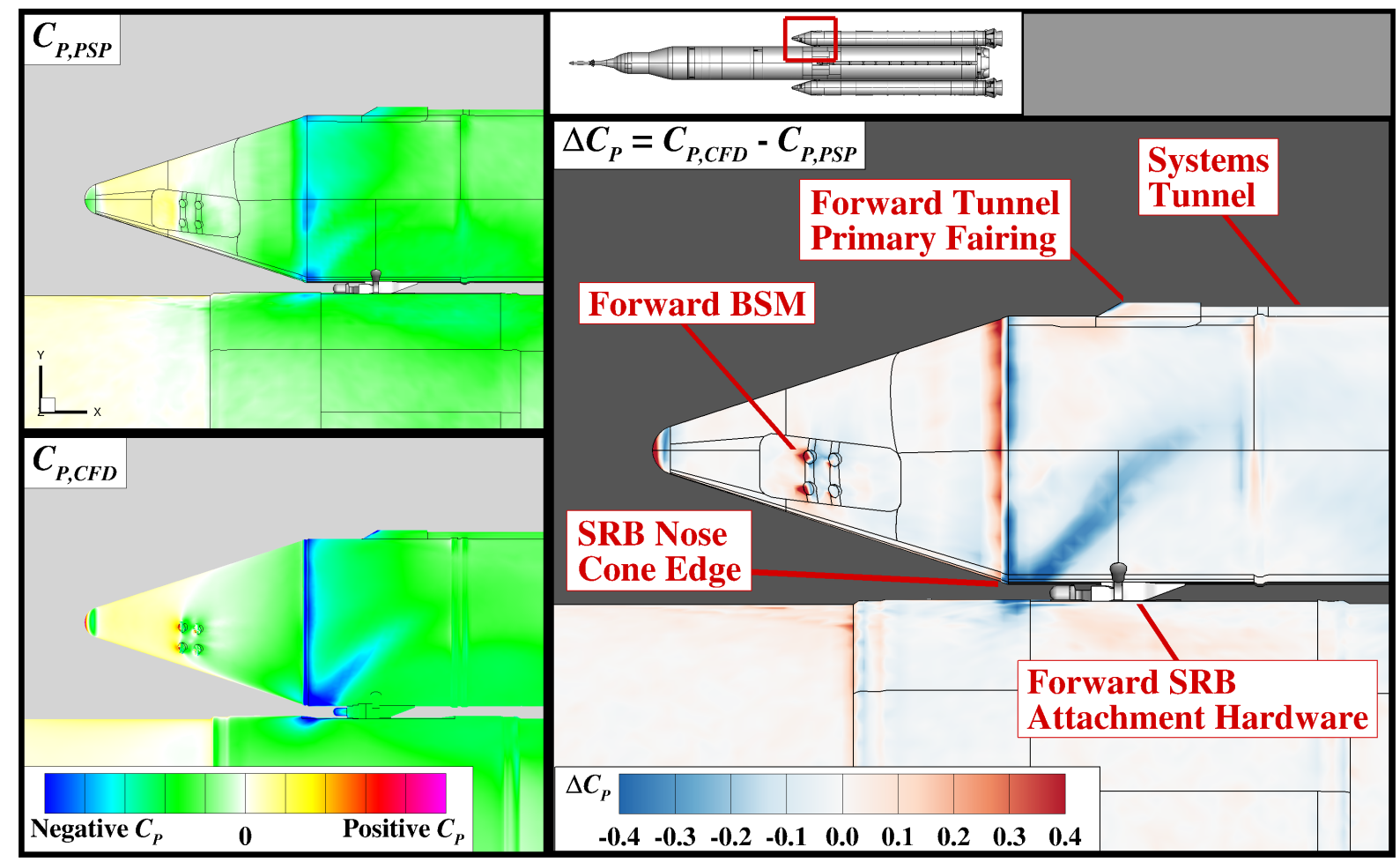

(a) Upper surface.

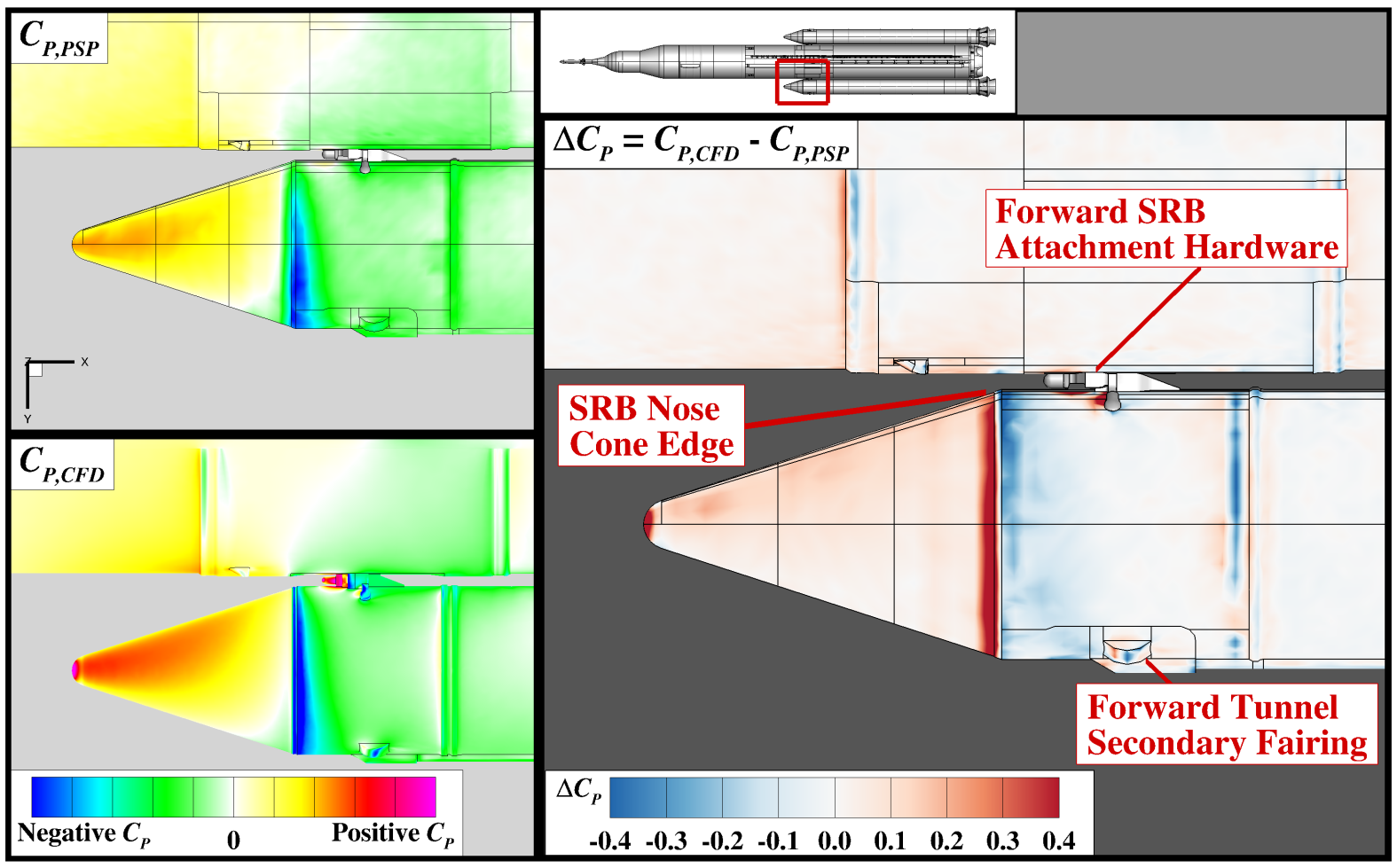

(b) Lower surface.

Fig. 21 Comparison of PSP and CFD surface pressure coefficient data on the starboard forward SRB of the SLS-28k configuration at $M_{\infty}=0.8, \alpha_{V}=8^{\circ}$, and $\phi_{V}=0^{\circ}$. 


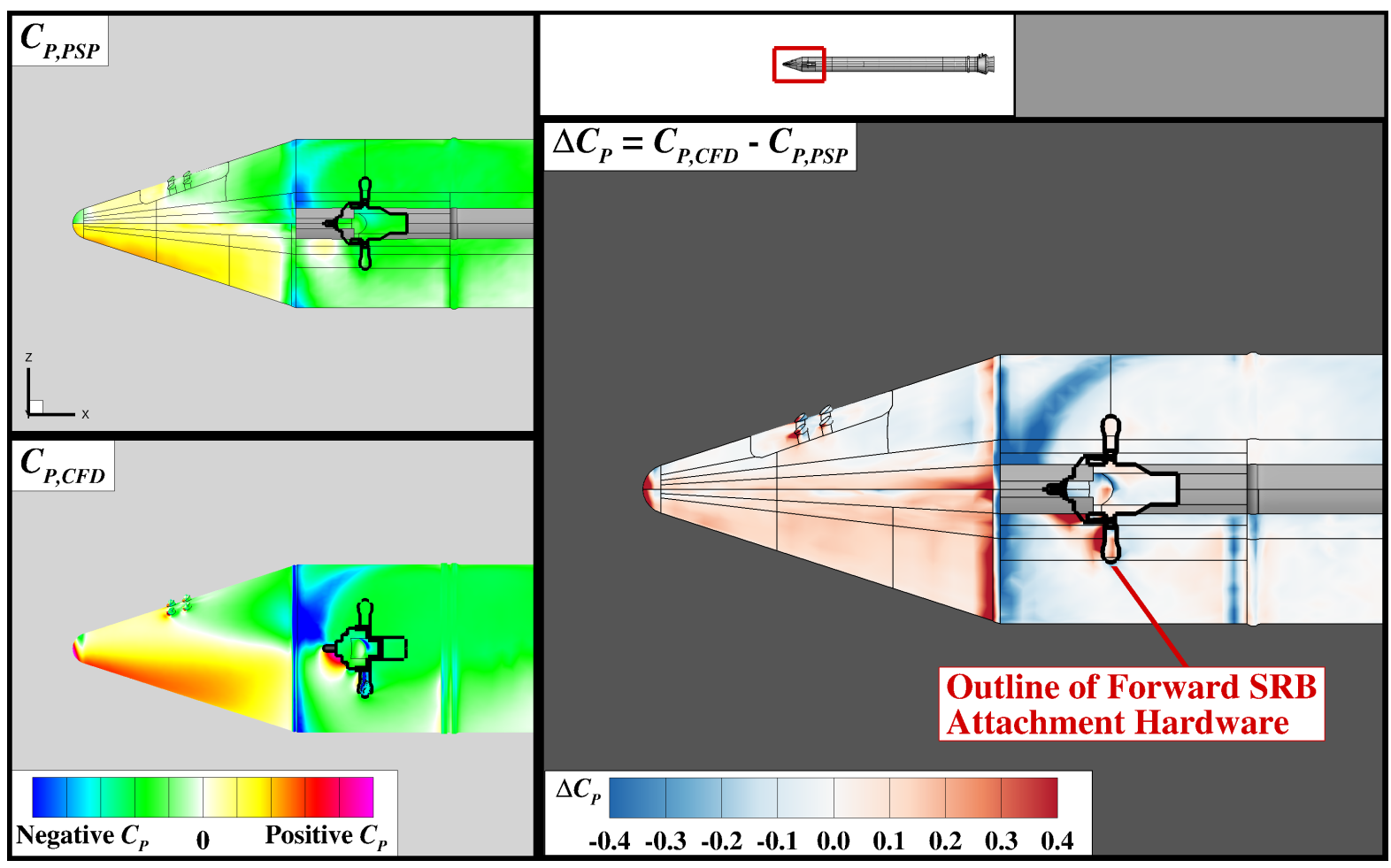

Fig. 22 Comparison of PSP and CFD surface pressure coefficient data for the inboard side of the starboard forward SRB of the SLS-28k configuration at $M_{\infty}=0.8, \alpha_{V}=8^{\circ}$, and $\phi_{V}=0^{\circ}$.

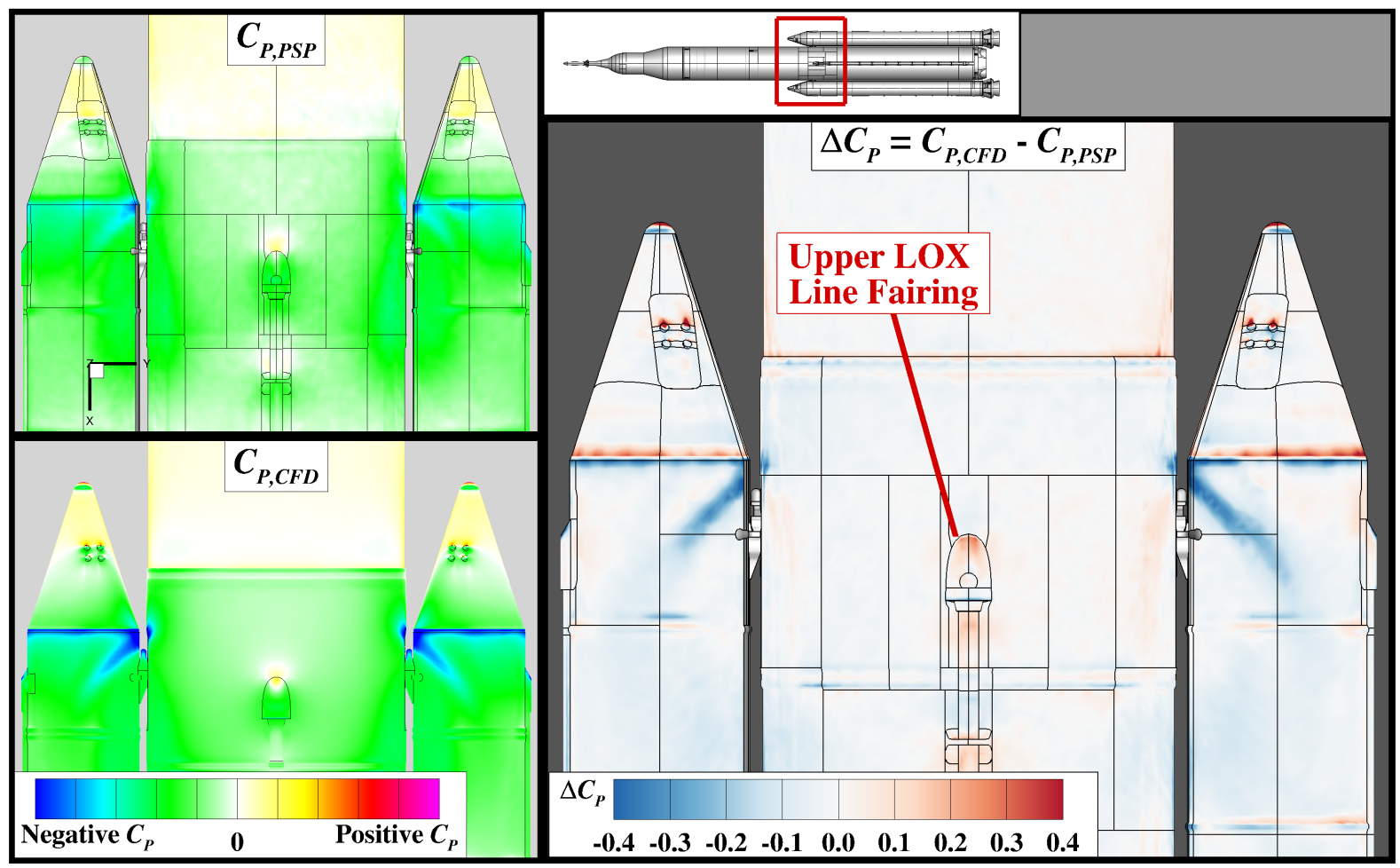

(a) Upper surface.

Fig. 23 Comparison of PSP and CFD surface pressure coefficient data near the forward SRB attachment point of the SLS-28k configuration at $M_{\infty}=0.8, \alpha_{V}=8^{\circ}$, and $\phi_{V}=0^{\circ}$. 


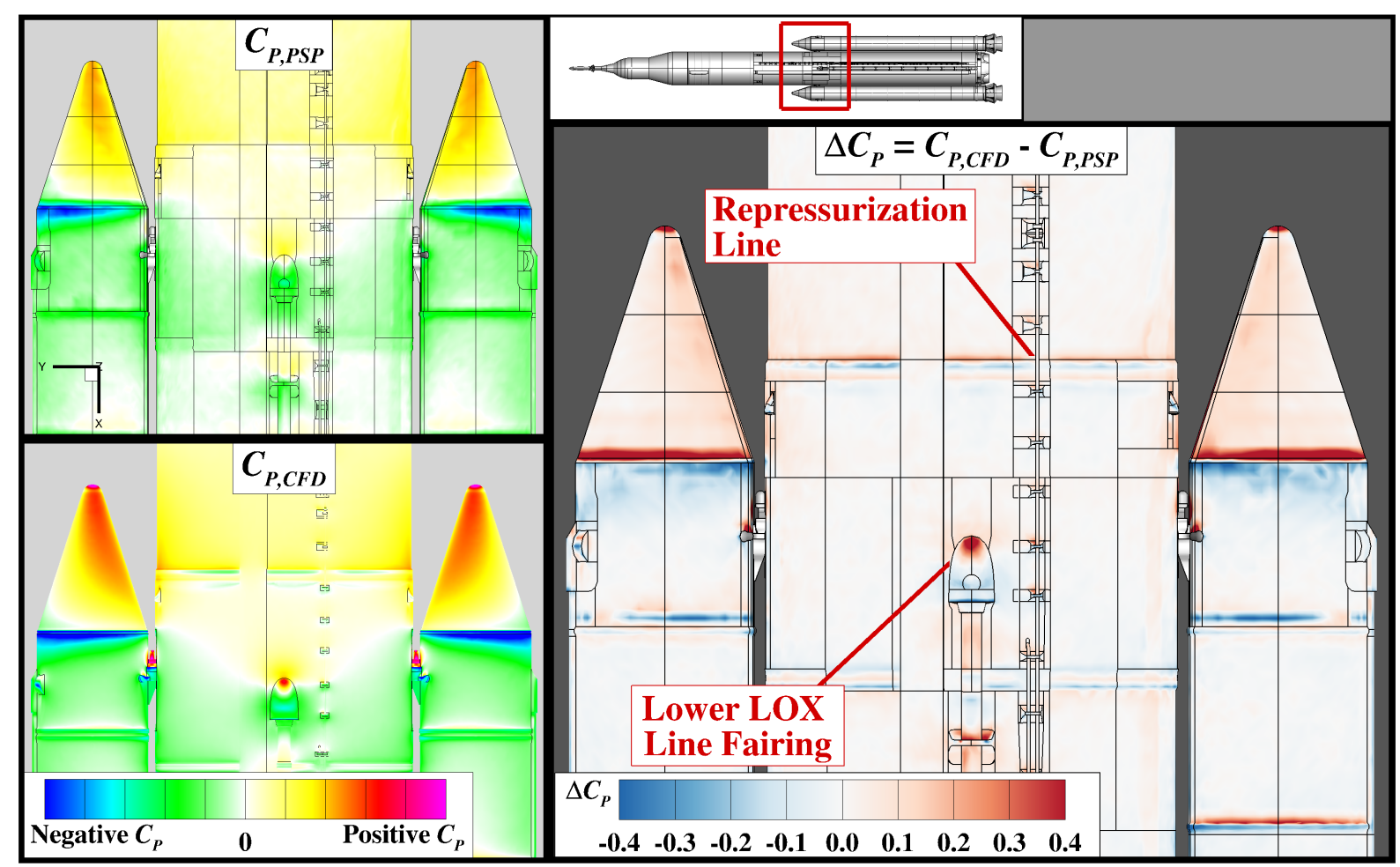

(b) Lower surface.

Fig. 23 Concluded.

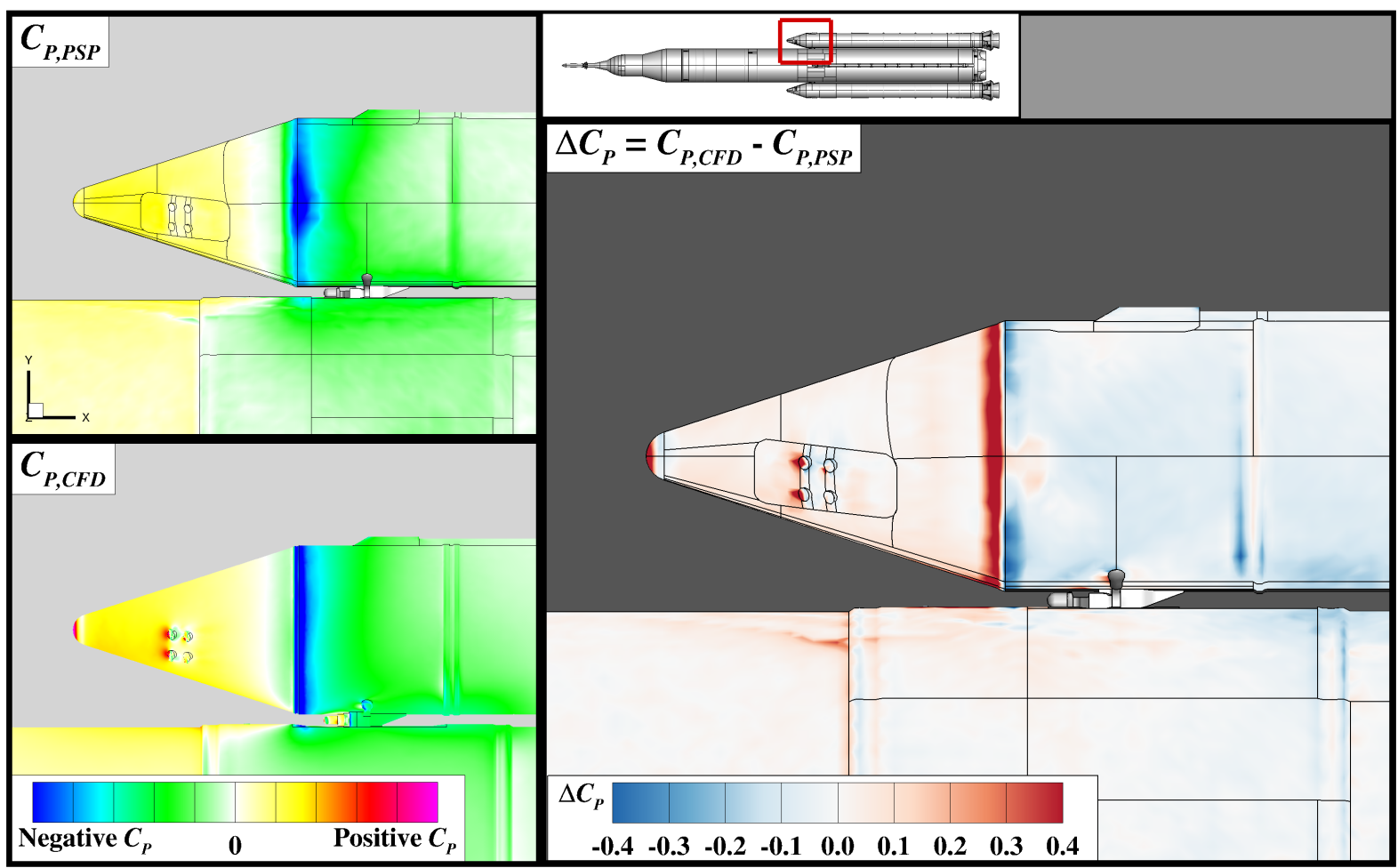

(a) Upper surface.

Fig. 24 Comparison of PSP and CFD surface pressure coefficient data on the starboard forward SRB of the SLS-28k configuration at $M_{\infty}=0.8, \alpha_{V}=0^{\circ}$, and $\phi_{V}=0^{\circ}$. 


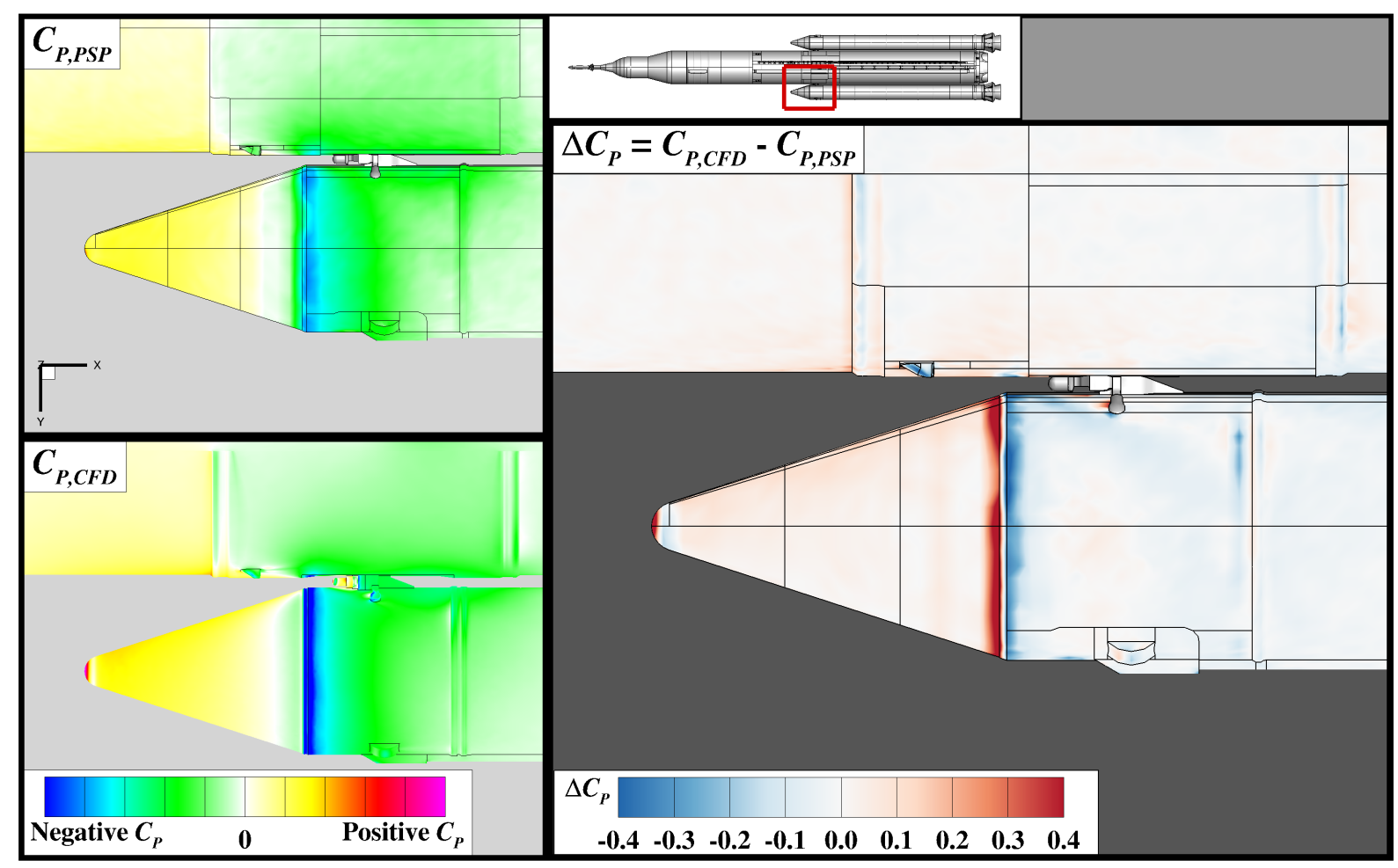

(b) Lower surface.

Fig. 24 Concluded.

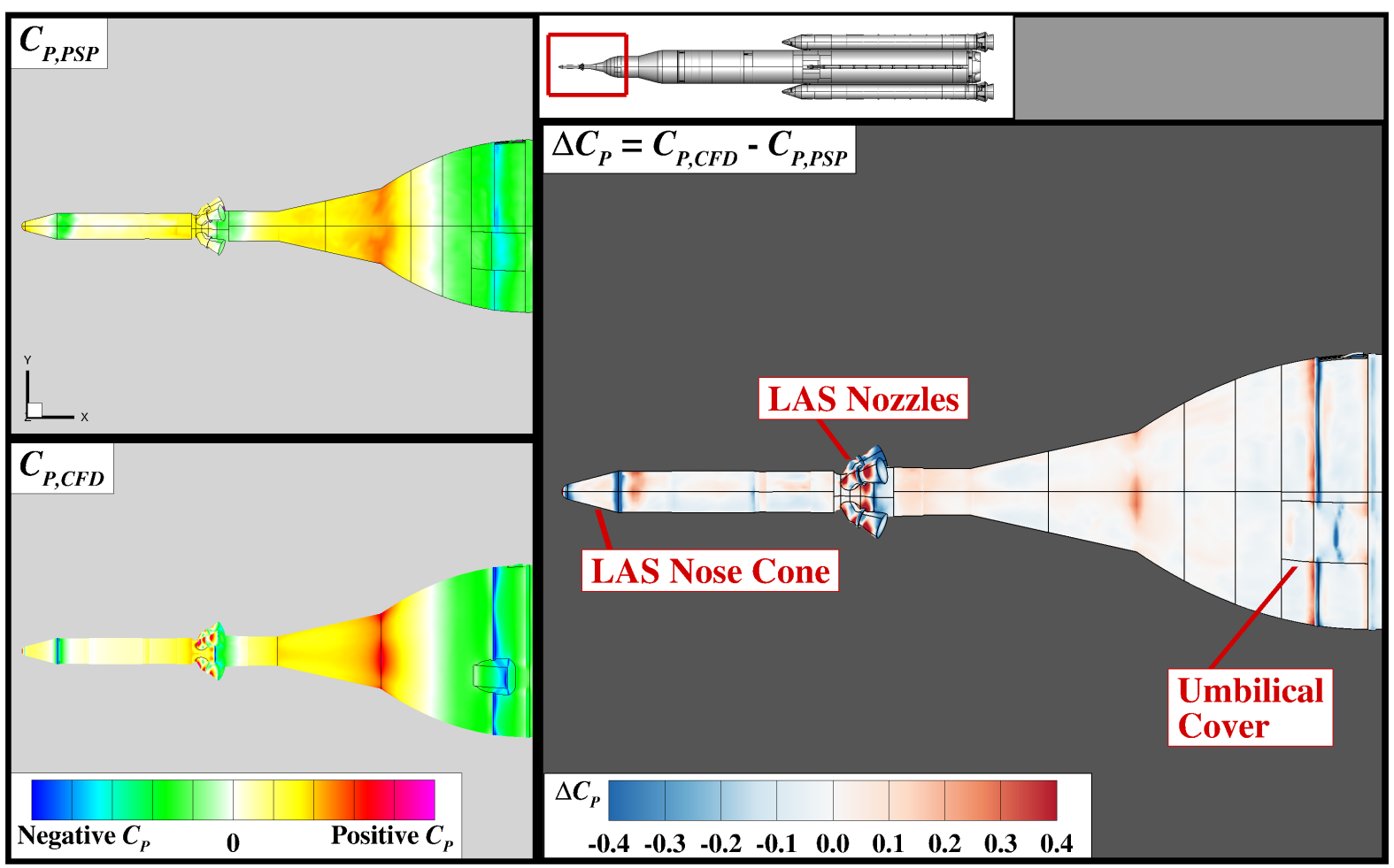

(a) Upper surface.

Fig. 25 Comparison of PSP and CFD surface pressure coefficient data on the LAS/MPCV of the SLS-28k configuration at $M_{\infty}=0.8, \alpha_{V}=8^{\circ}$, and $\phi_{V}=0^{\circ}$. 


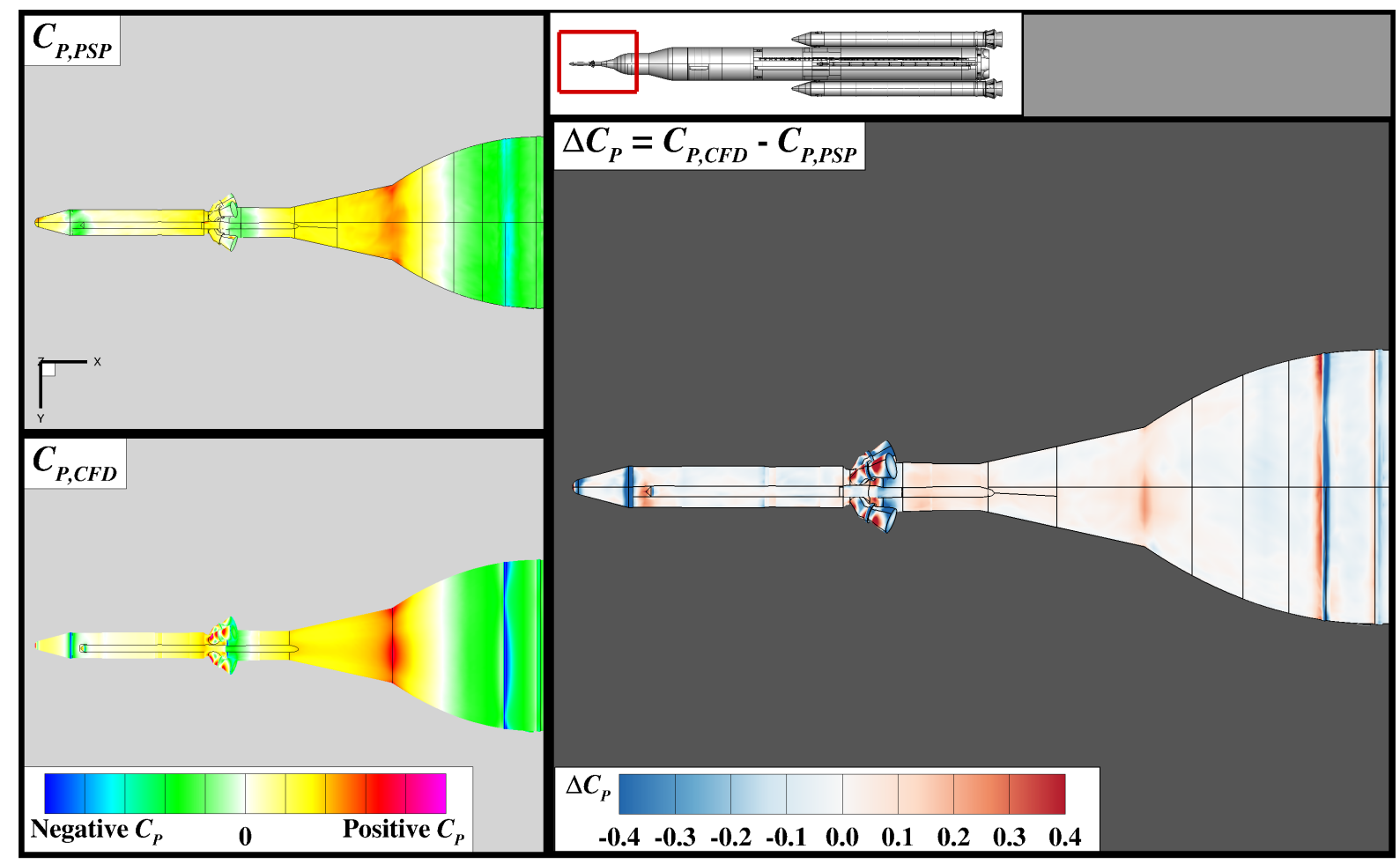

(b) Lower surface.

Fig. 25 Concluded.

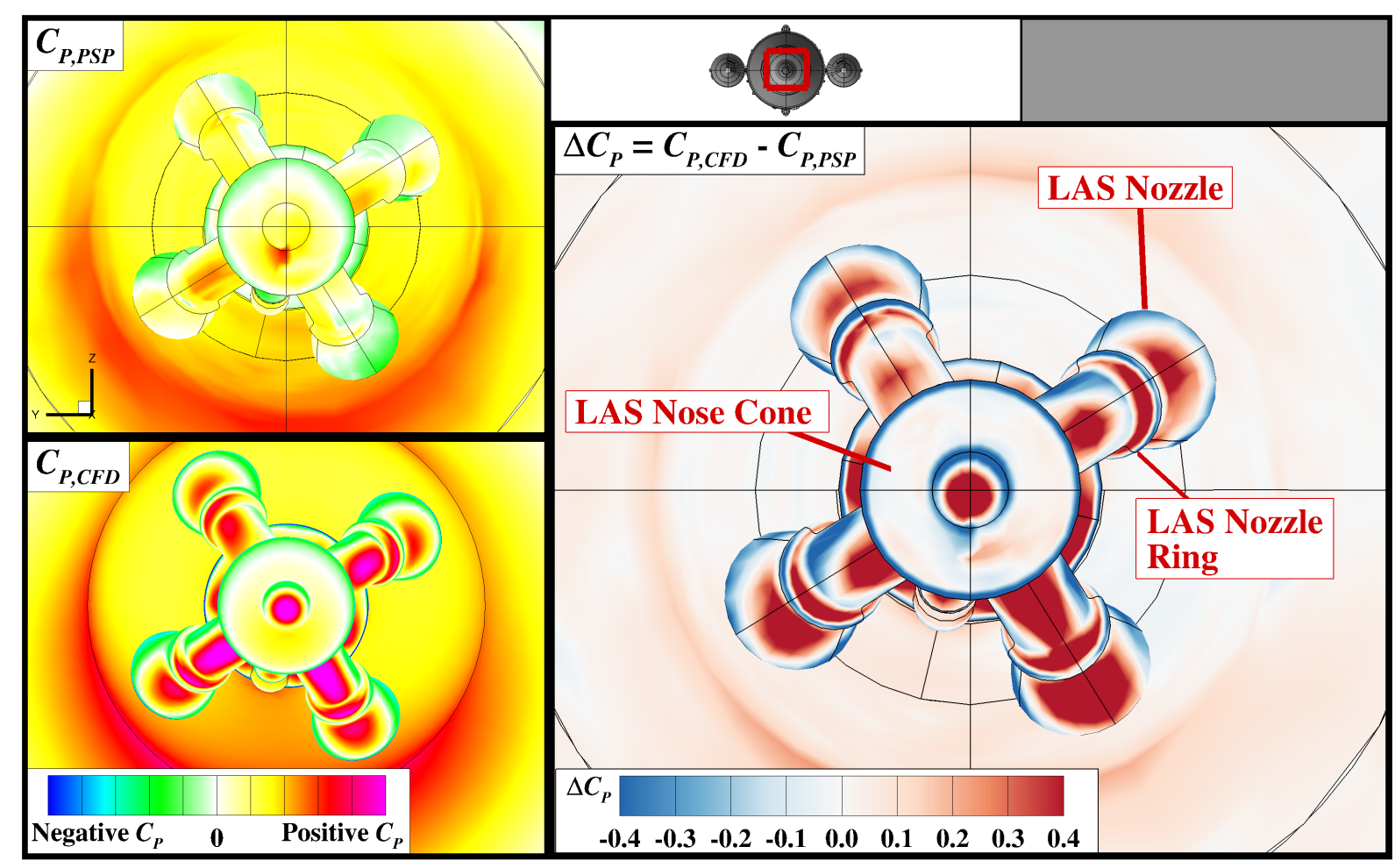

Fig. 26 Comparison of PSP and CFD surface pressure coefficient data on the LAS tower from a front view of the SLS-28k configuration at $M_{\infty}=0.8, \alpha_{V}=8^{\circ}$, and $\phi_{V}=0^{\circ}$. 


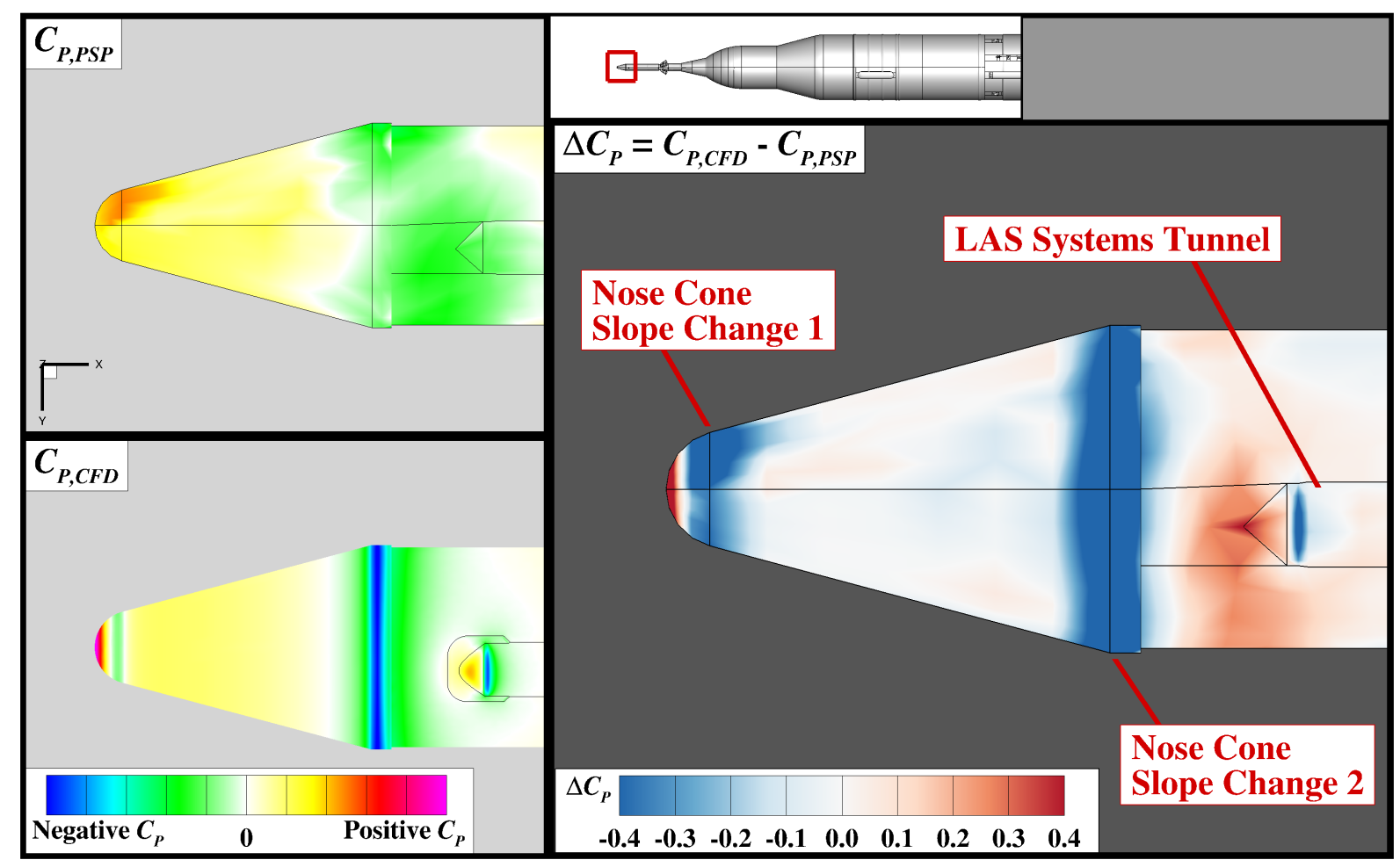

Fig. 27 Comparison of PSP and CFD surface pressure coefficient data on the lower LAS nose surface of the SLS-28k configuration at $M_{\infty}=0.8, \alpha_{V}=8^{\circ}$, and $\phi_{V}=0^{\circ}$.

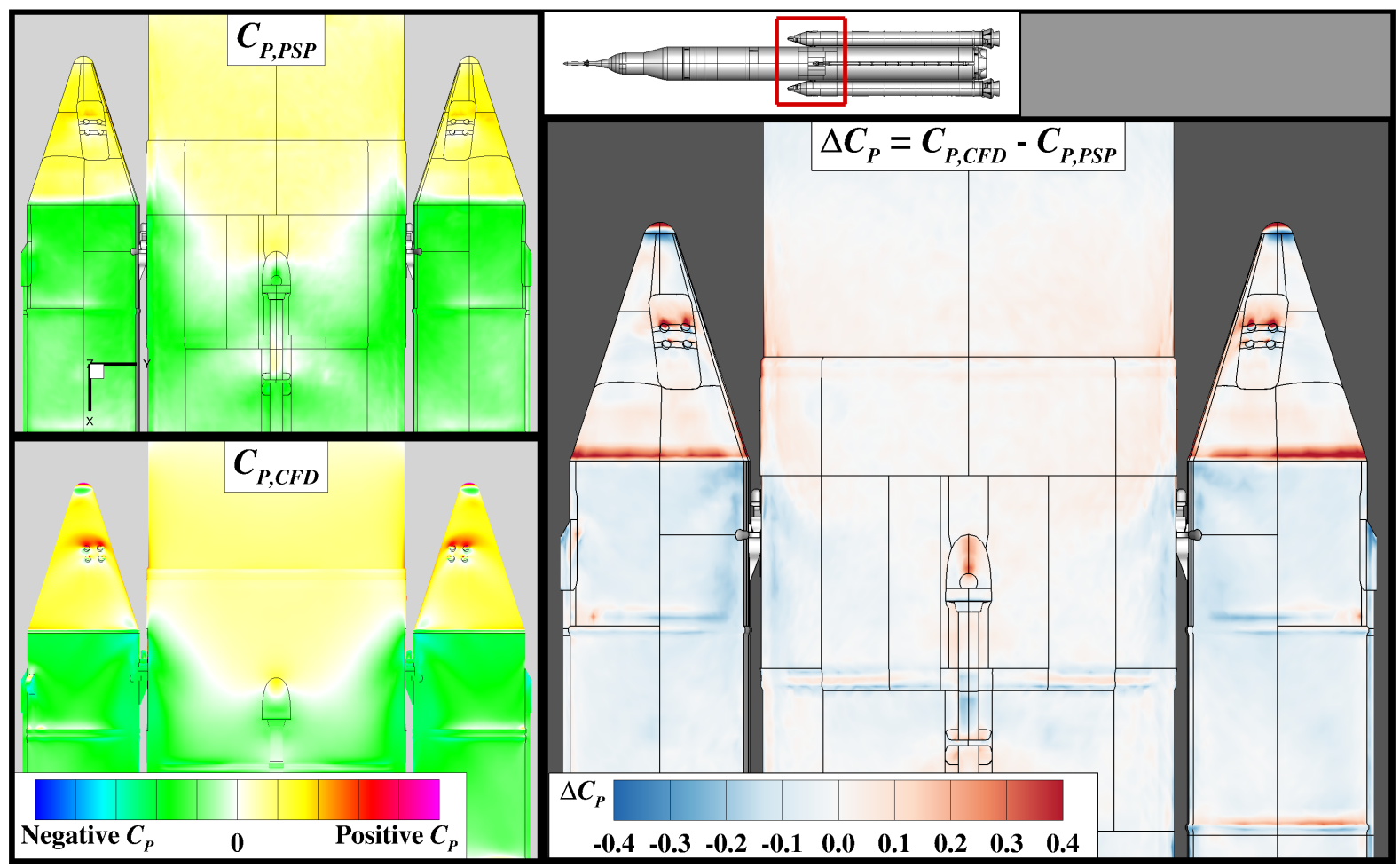

(a) Upper surface.

Fig. 28 Comparison of PSP and CFD surface pressure coefficient data near the forward SRB attachment point of the SLS-28k configuration at $M_{\infty}=1.3, \alpha_{V}=8^{\circ}$, and $\phi_{V}=0^{\circ}$. 


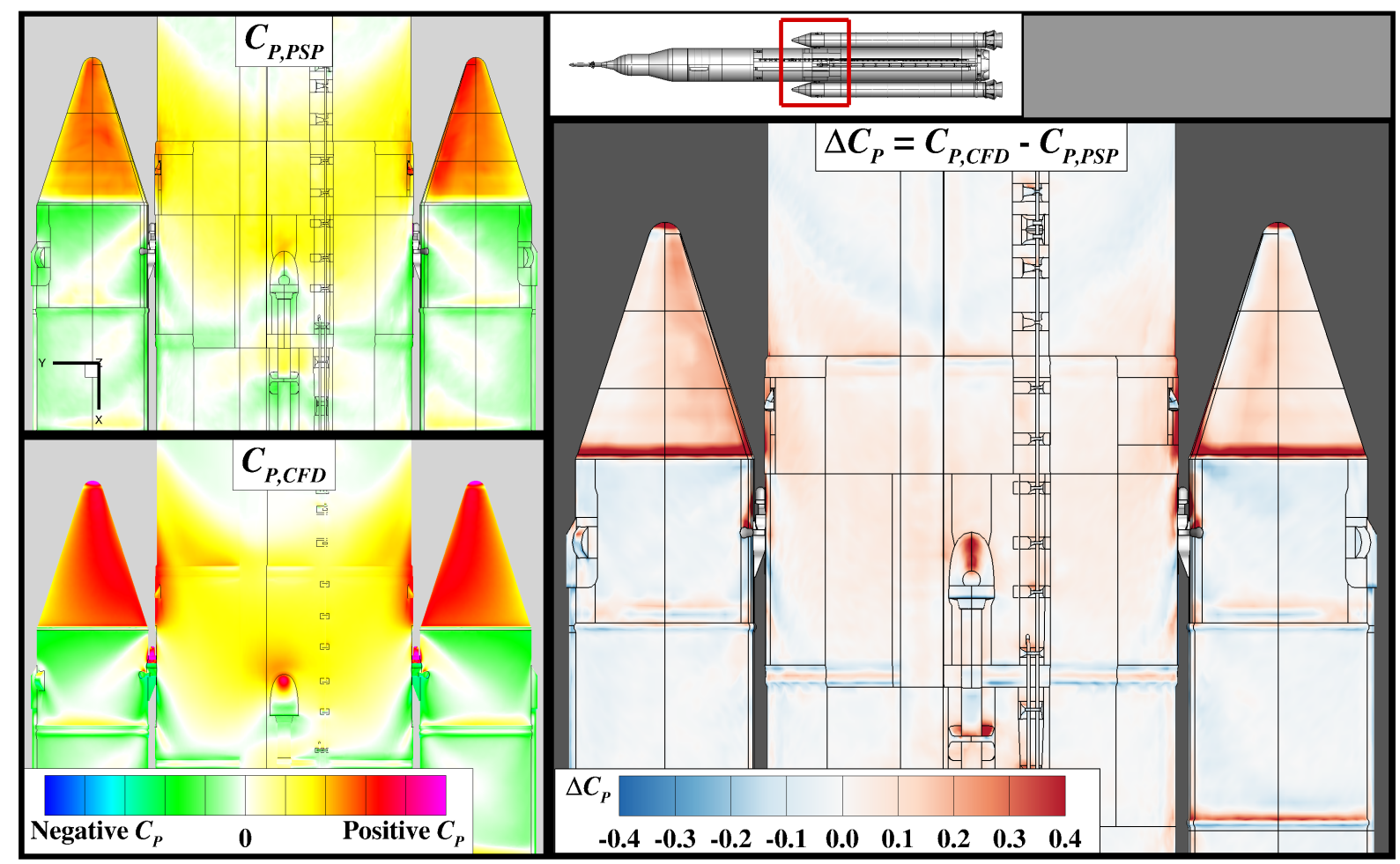

(b) Lower surface.

Fig. 28 Concluded.

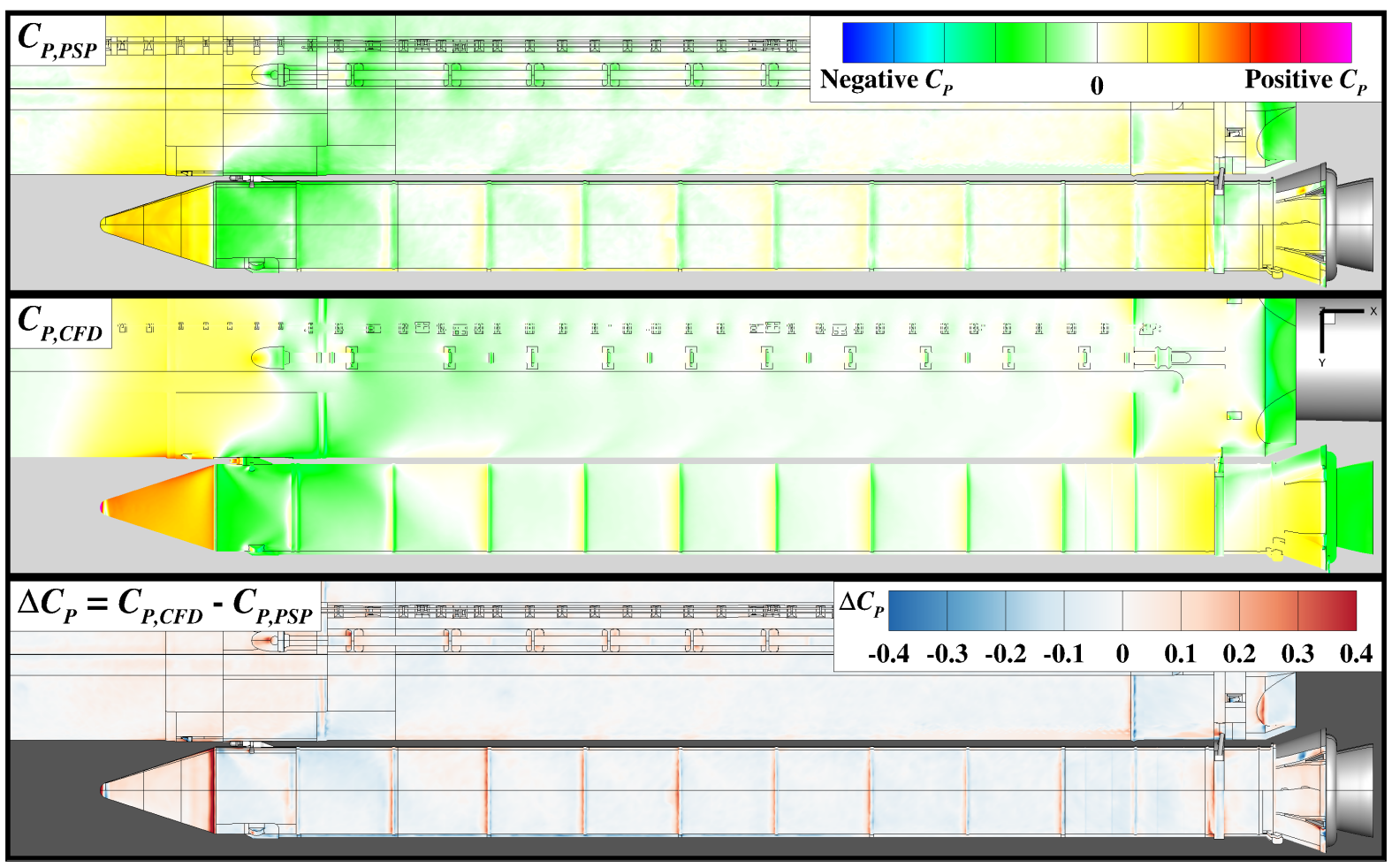

(a) $\alpha_{V}=0^{\circ}$.

Fig. 29 Comparison of PSP and CFD surface pressure coefficient data on the lower surface of the starboard SRB of the SLS-28k configuration at $M_{\infty}=1.3, \alpha_{V}=0^{\circ}, 2^{\circ}, 4^{\circ}$, and $8^{\circ}$, and $\phi_{V}=0^{\circ}$. 


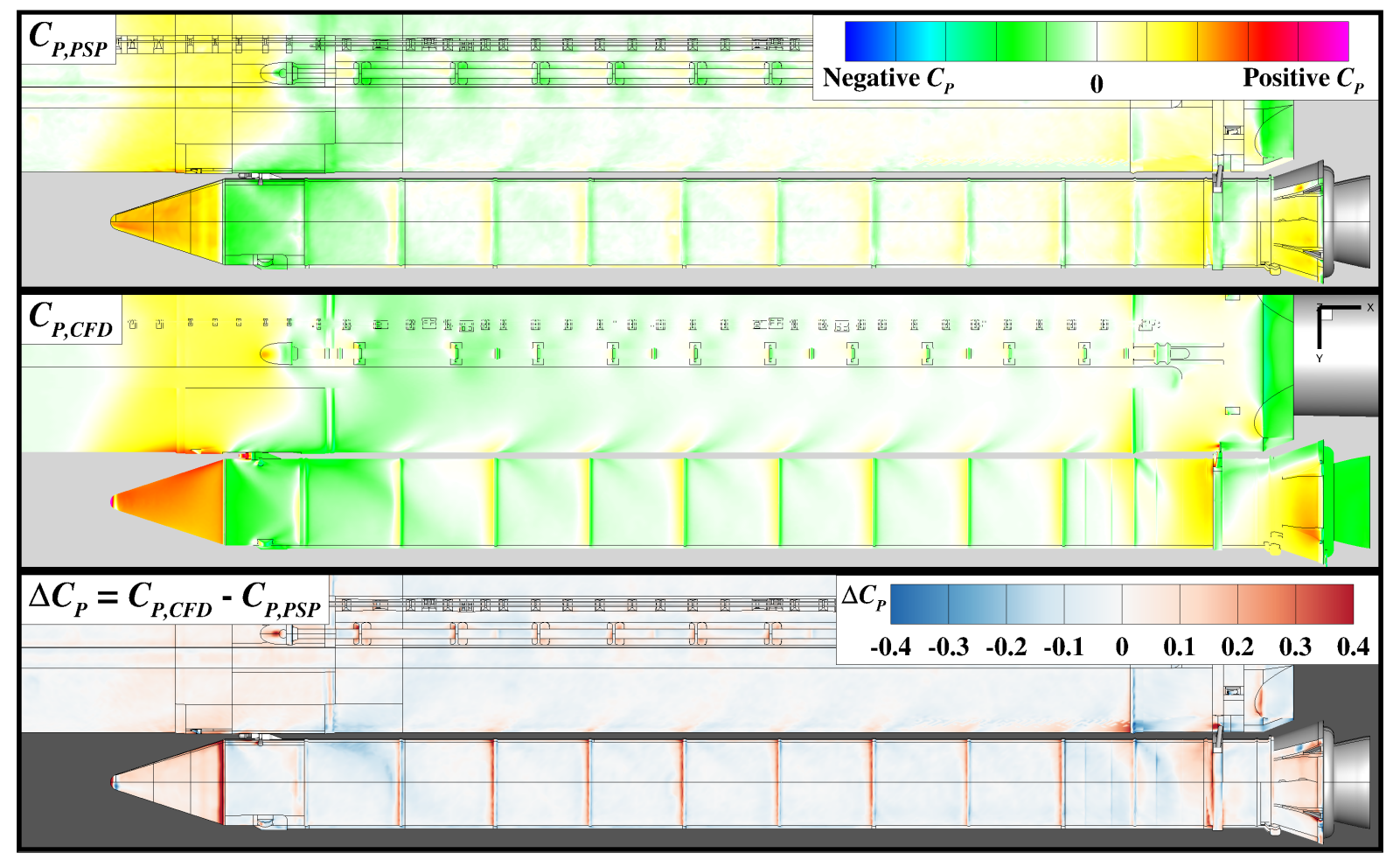

(b) $\alpha_{V}=2^{\circ}$.

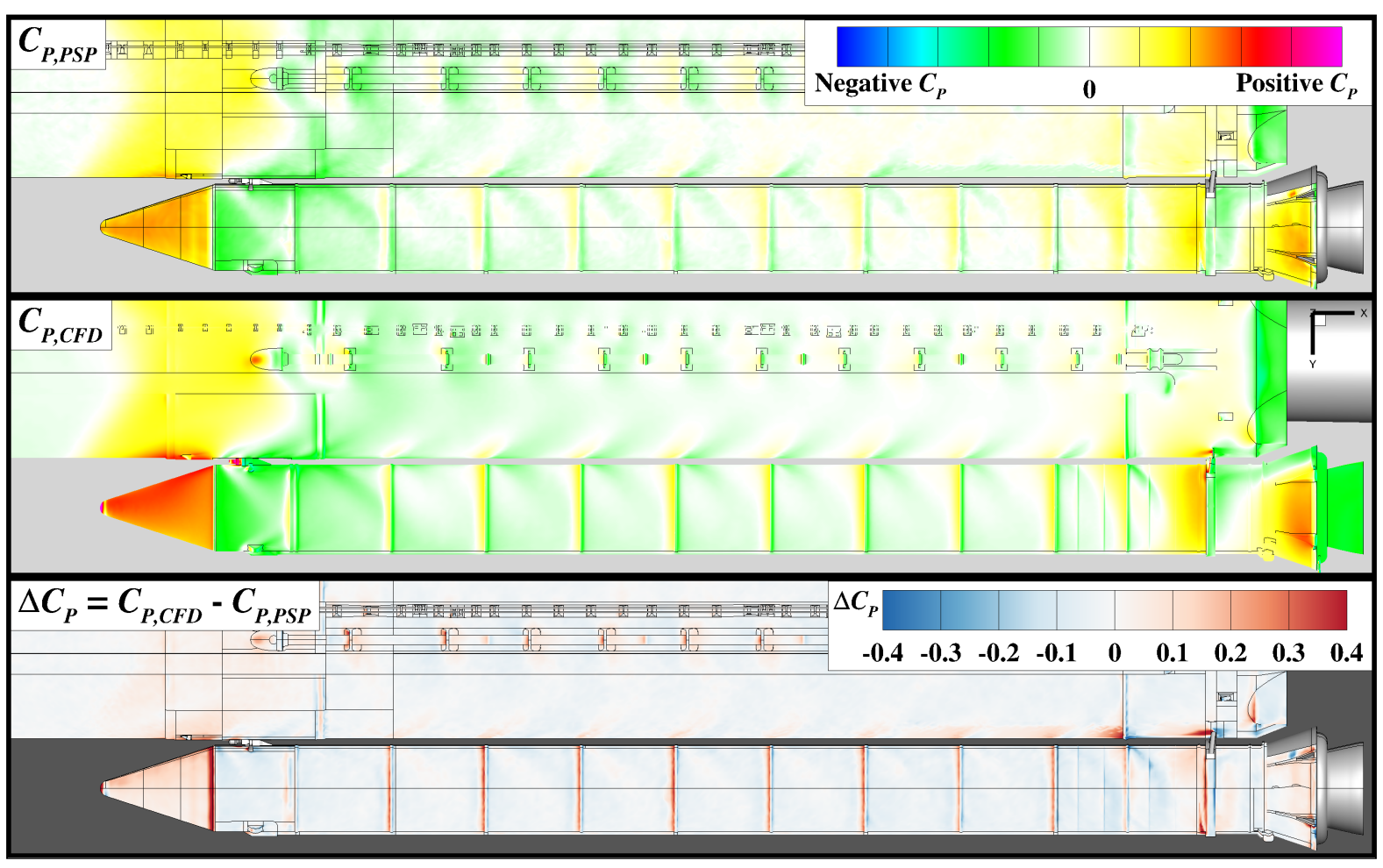

(c) $\alpha_{V}=4^{\circ}$.

Fig. 29 Continued. 


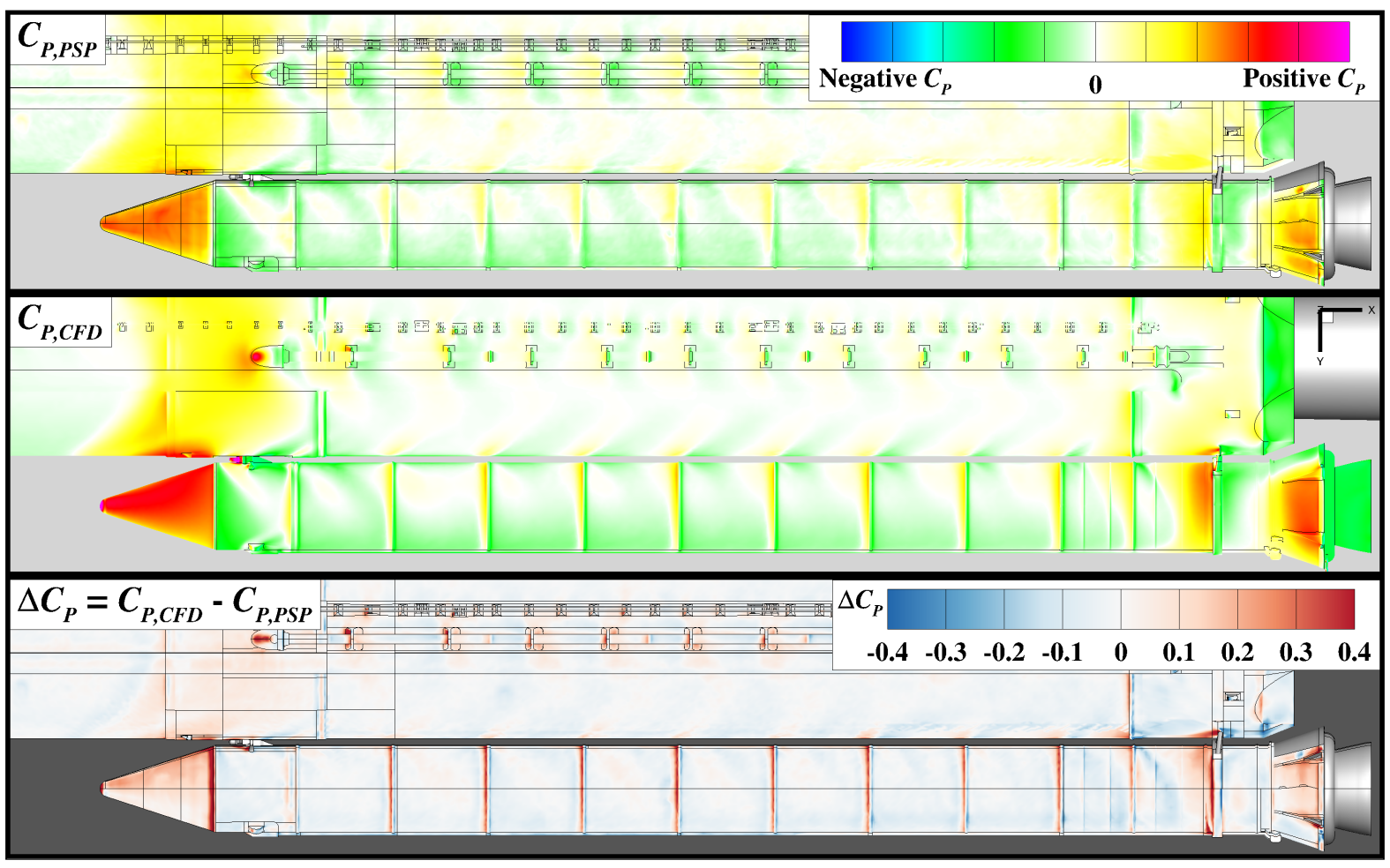

(d) $\alpha_{V}=\mathbf{8}^{\circ}$.

Fig. 29 Concluded.

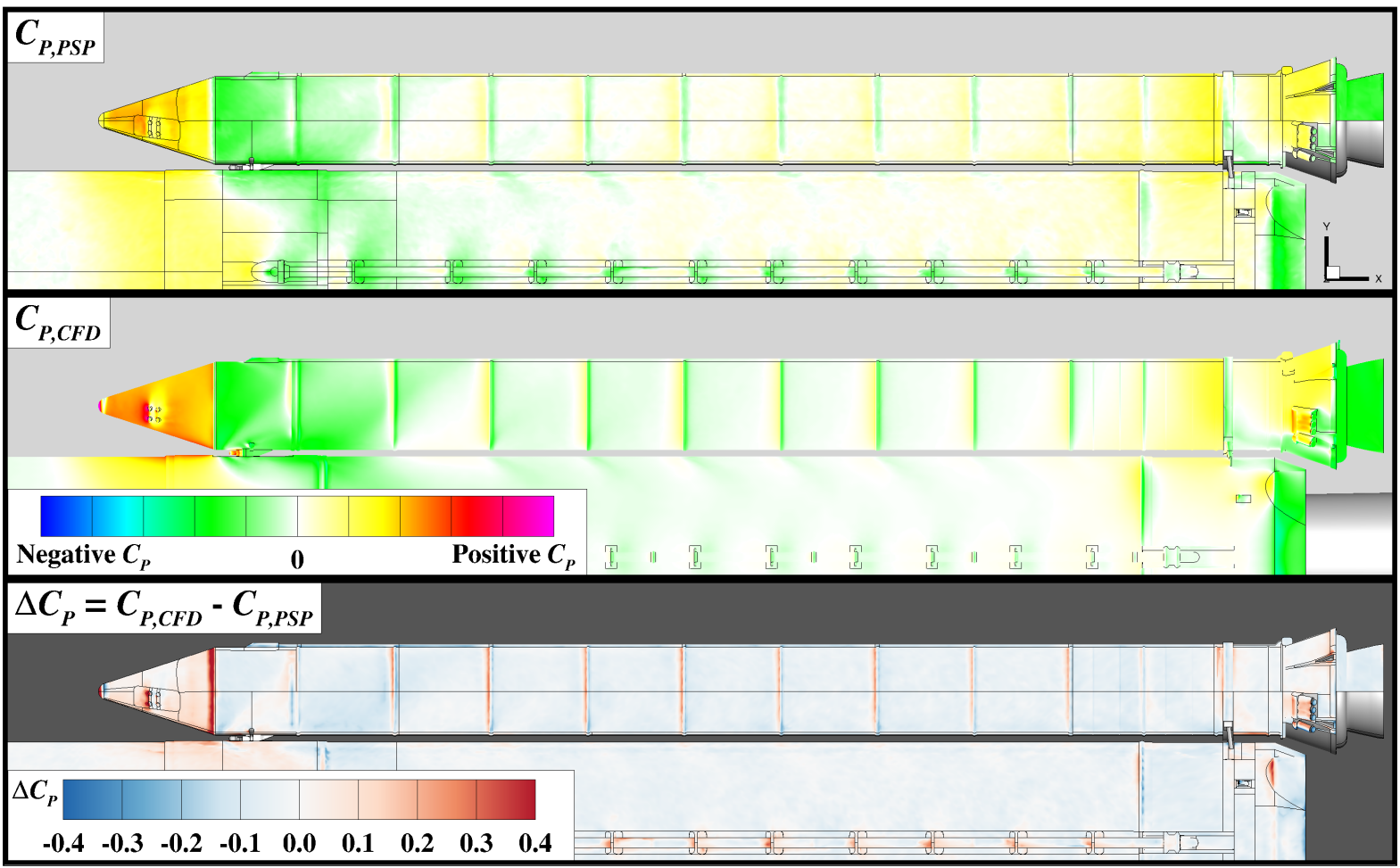

(a) $\alpha_{V}=\mathbf{0}^{\circ}$.

Fig. 30 Comparison of PSP and CFD surface pressure coefficient data on the upper surface of the starboard SRB of the SLS-28k configuration at $M_{\infty}=1.3, \alpha_{V}=0^{\circ}, 2^{\circ}, 4^{\circ}$, and $8^{\circ}$, and $\phi_{V}=0^{\circ}$. 


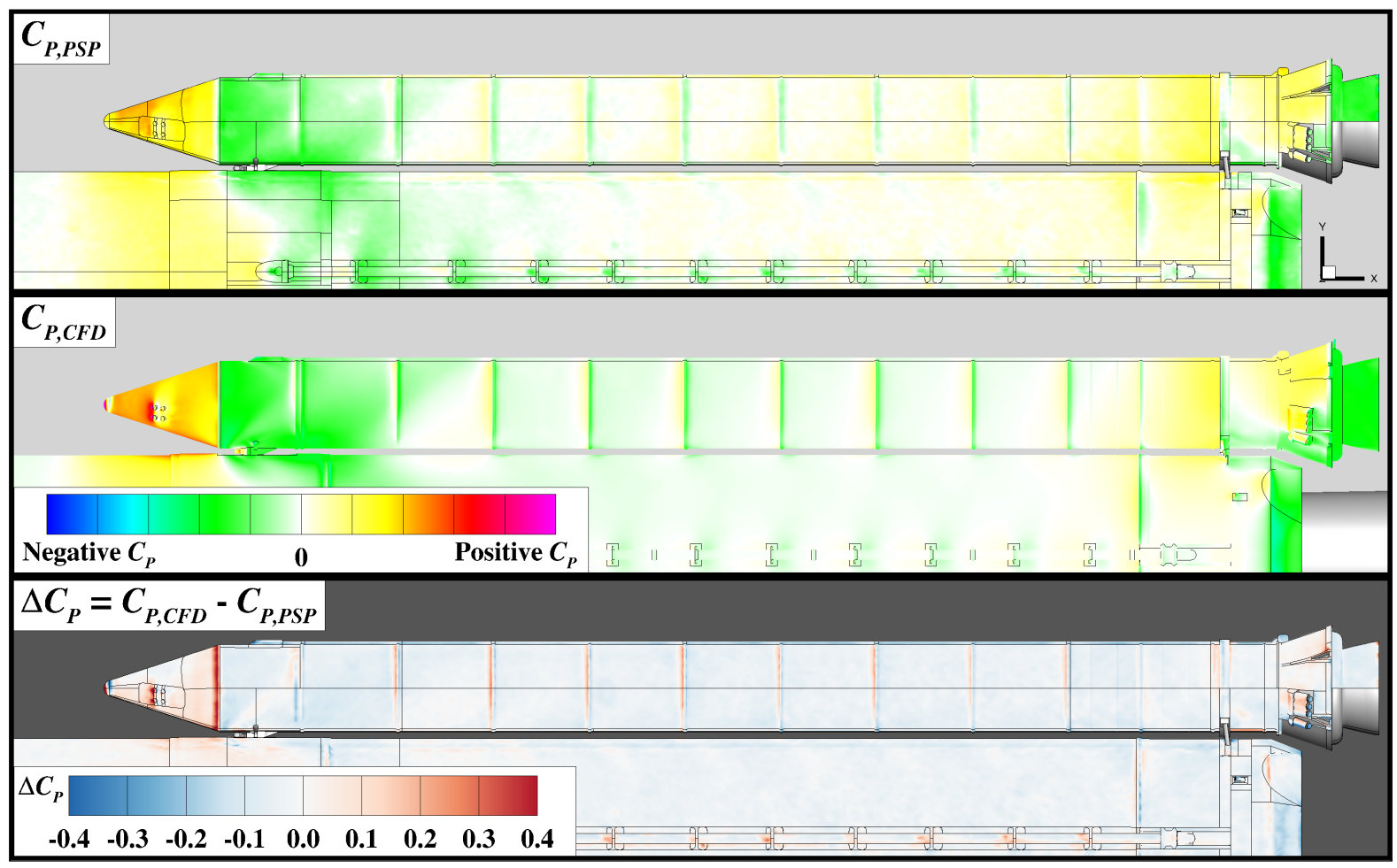

(b) $\alpha_{V}=2^{\circ}$.

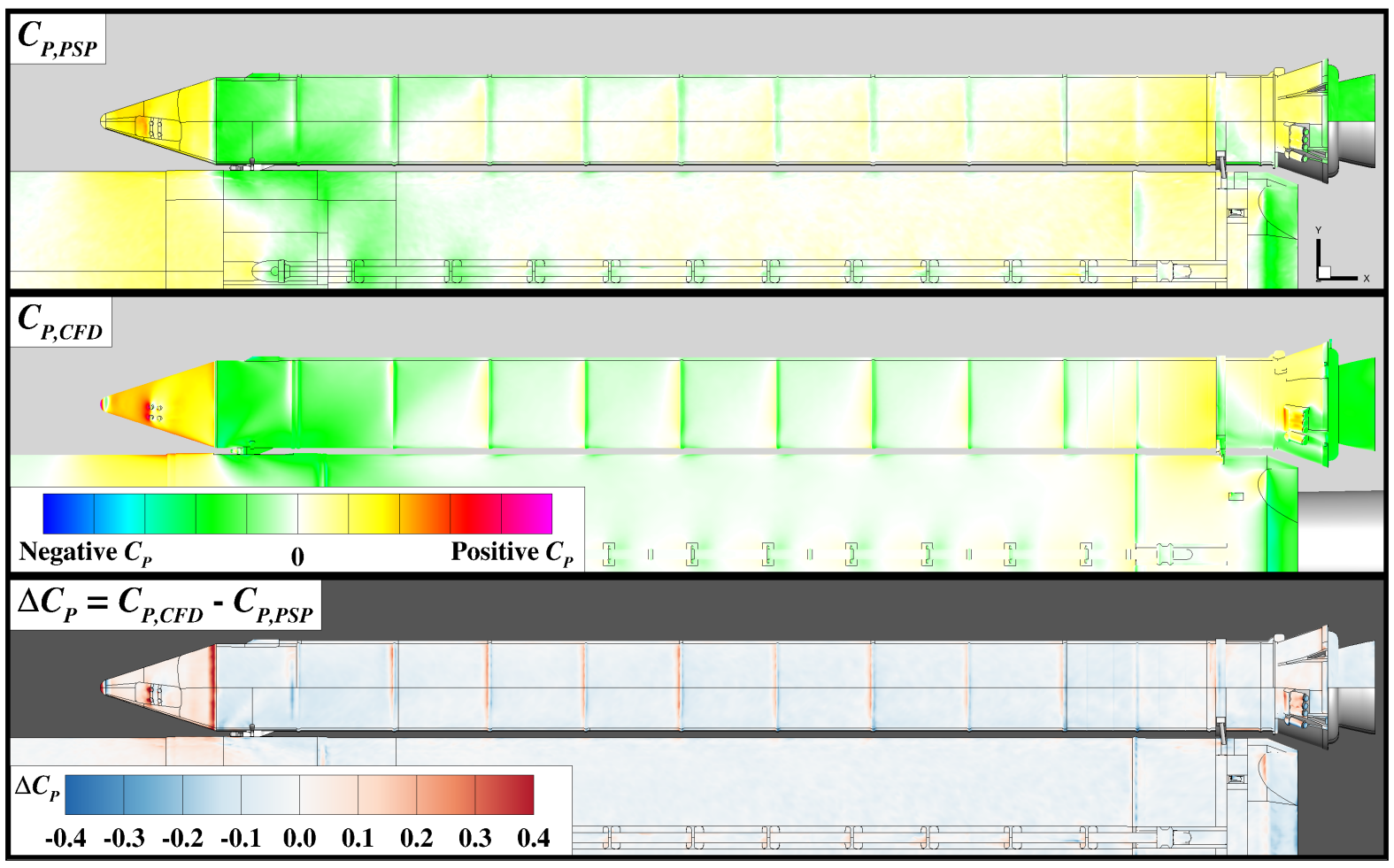

(c) $\alpha_{V}=4^{\circ}$.

Fig. 30 Continued. 


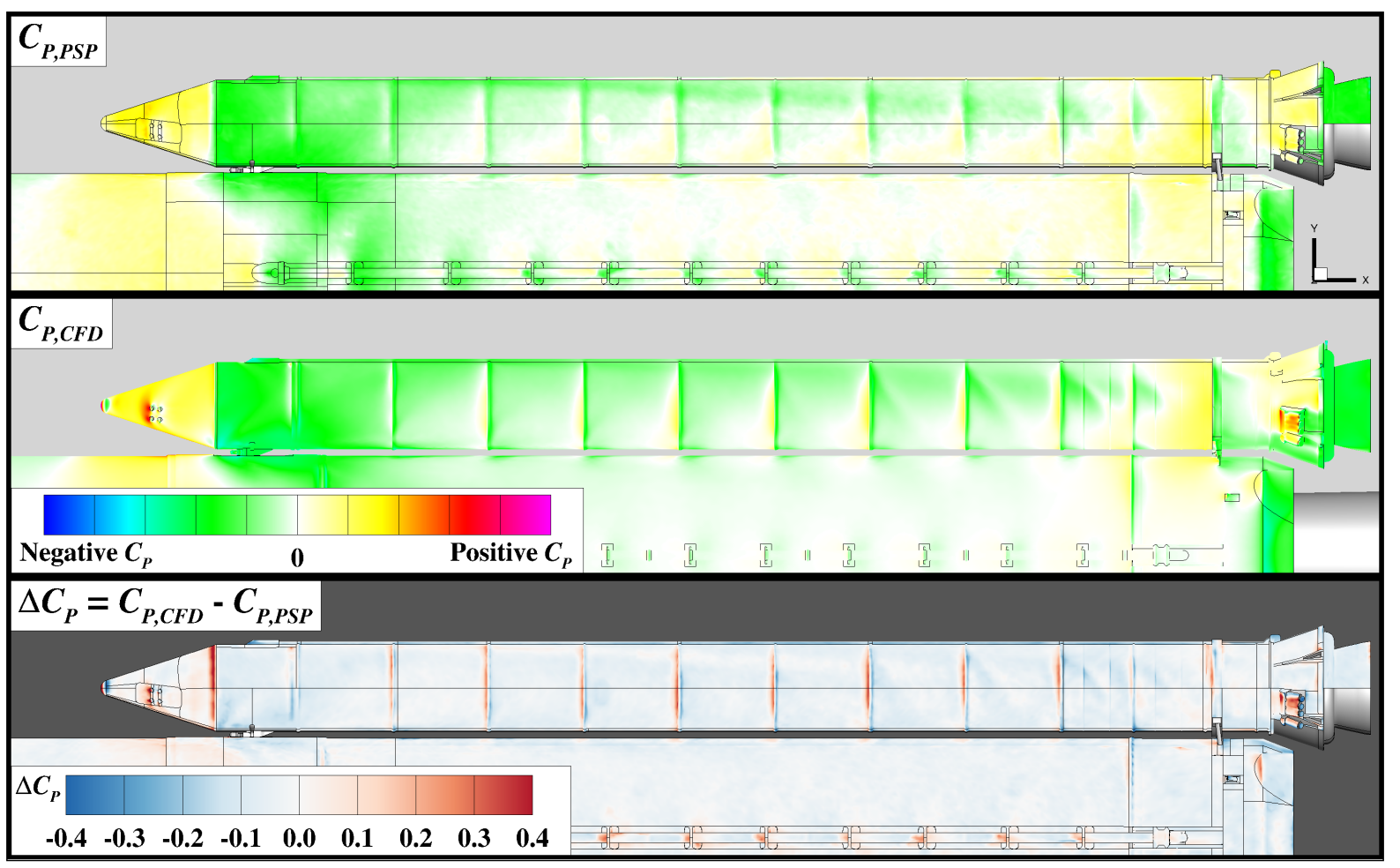

(d) $\alpha_{V}=8^{\circ}$.

Fig. 30 Concluded.

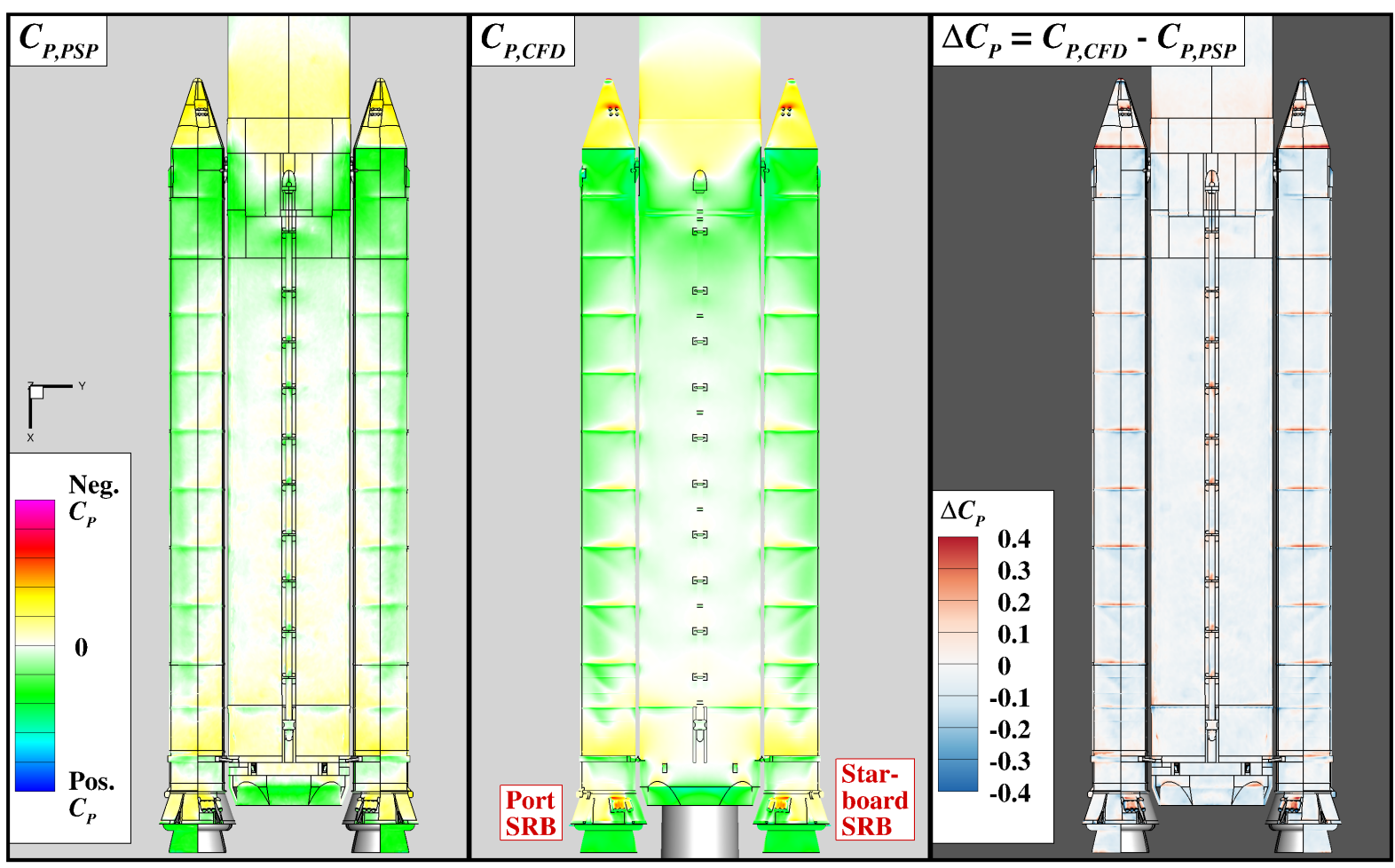

(a) $\phi_{V}=0^{\circ}$.

Fig. 31 Comparison of PSP and CFD surface pressure coefficient data on the upper surface of the core/SRBs of the SLS-28k configuration at $M_{\infty}=1.3, \alpha_{V}=8^{\circ}$, and $\phi_{V}=0^{\circ}, 4^{\circ}$, and $90^{\circ}$. 


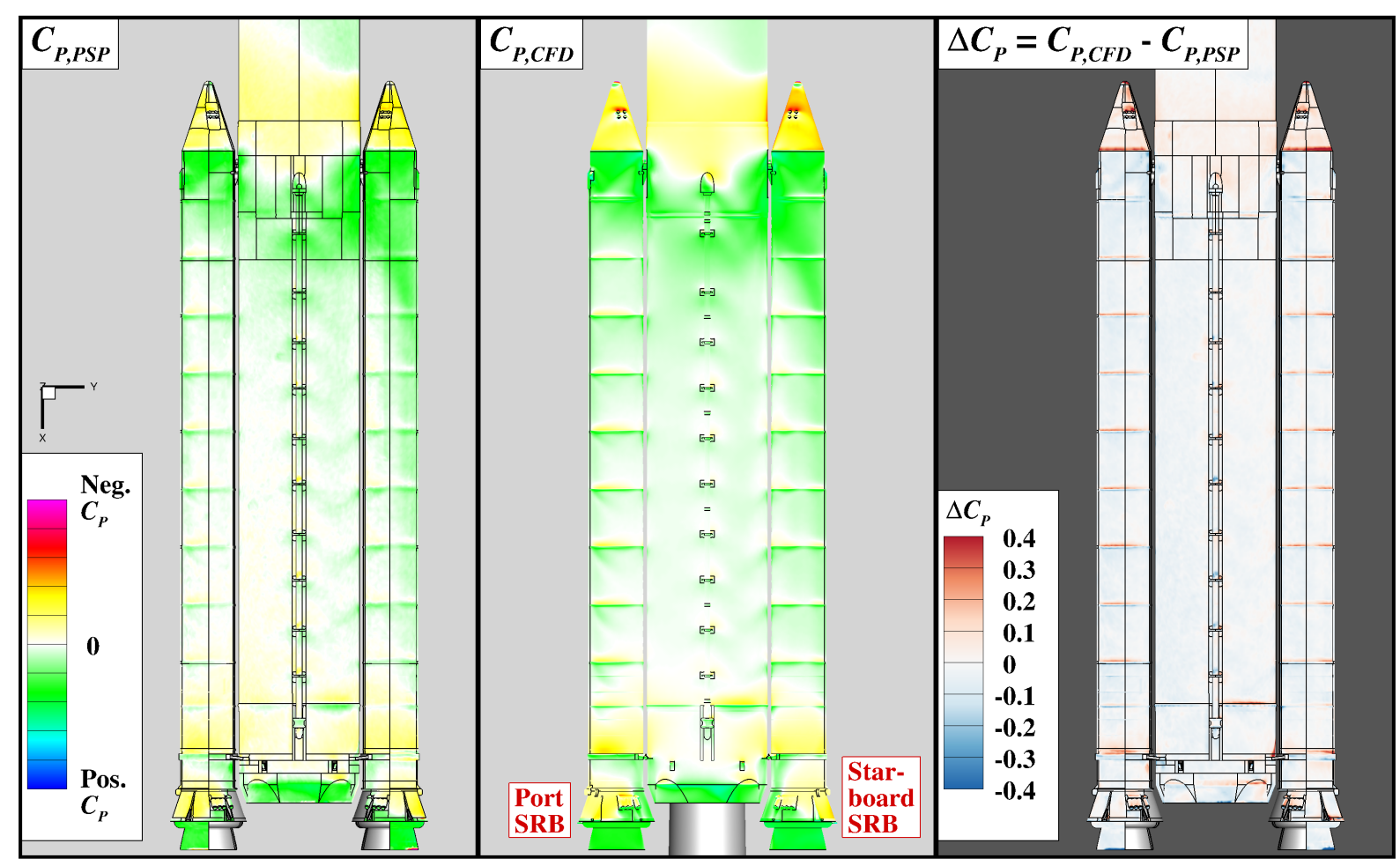

(b) $\phi_{V}=45^{\circ}$.

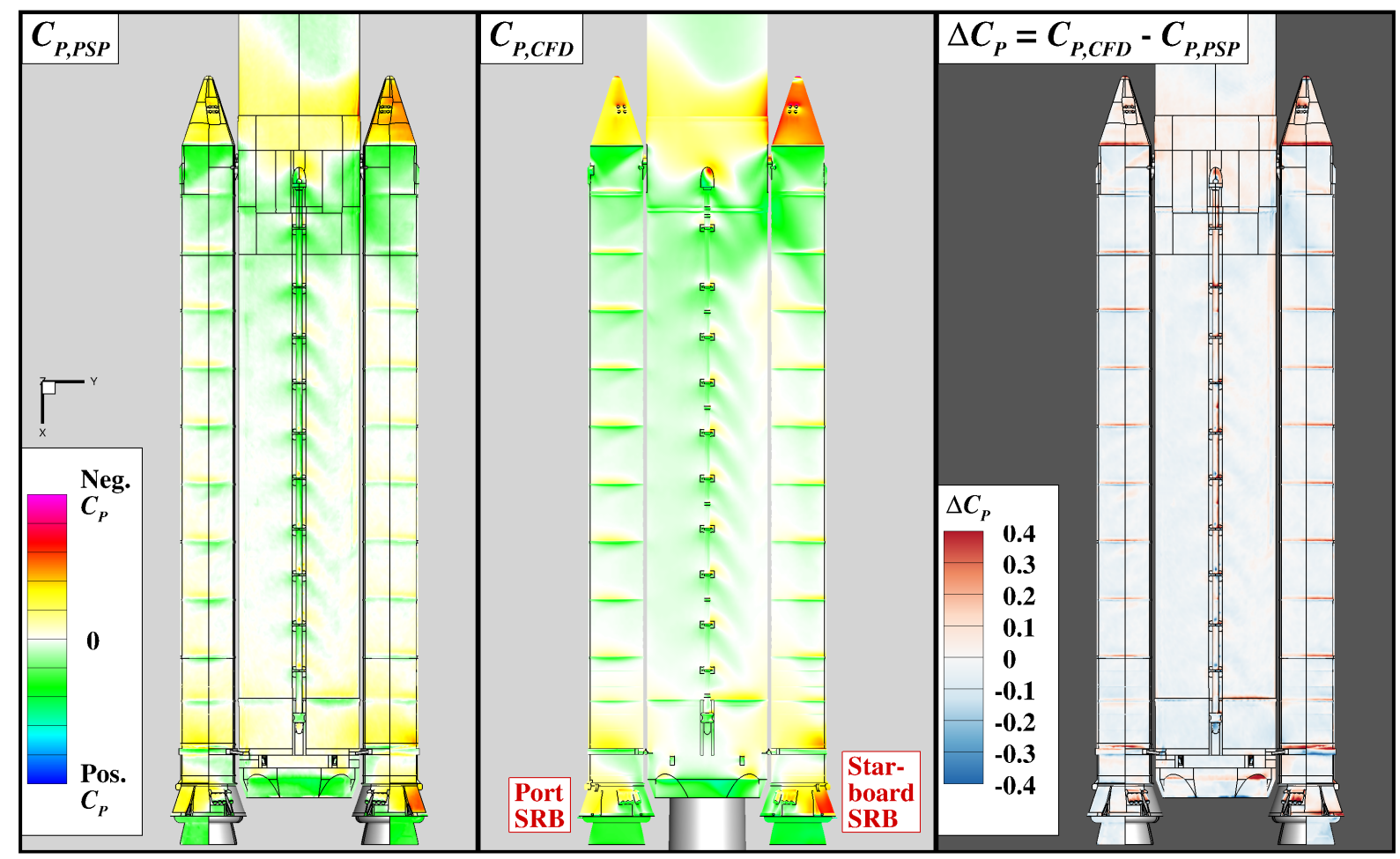

(c) $\phi_{V}=90^{\circ}$.

Fig. 31 Concluded. 


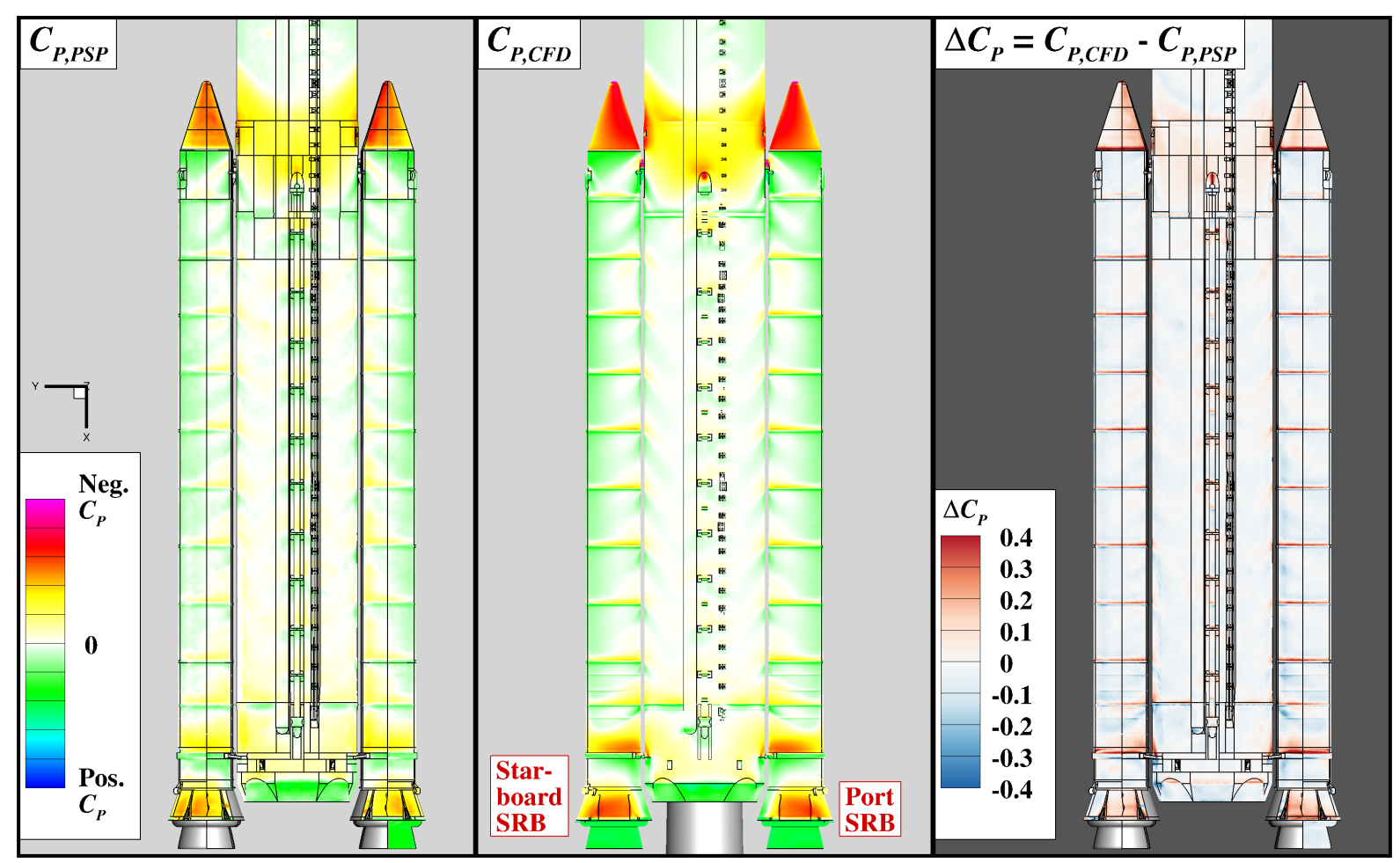

(a) $\phi_{V}=0^{\circ}$.

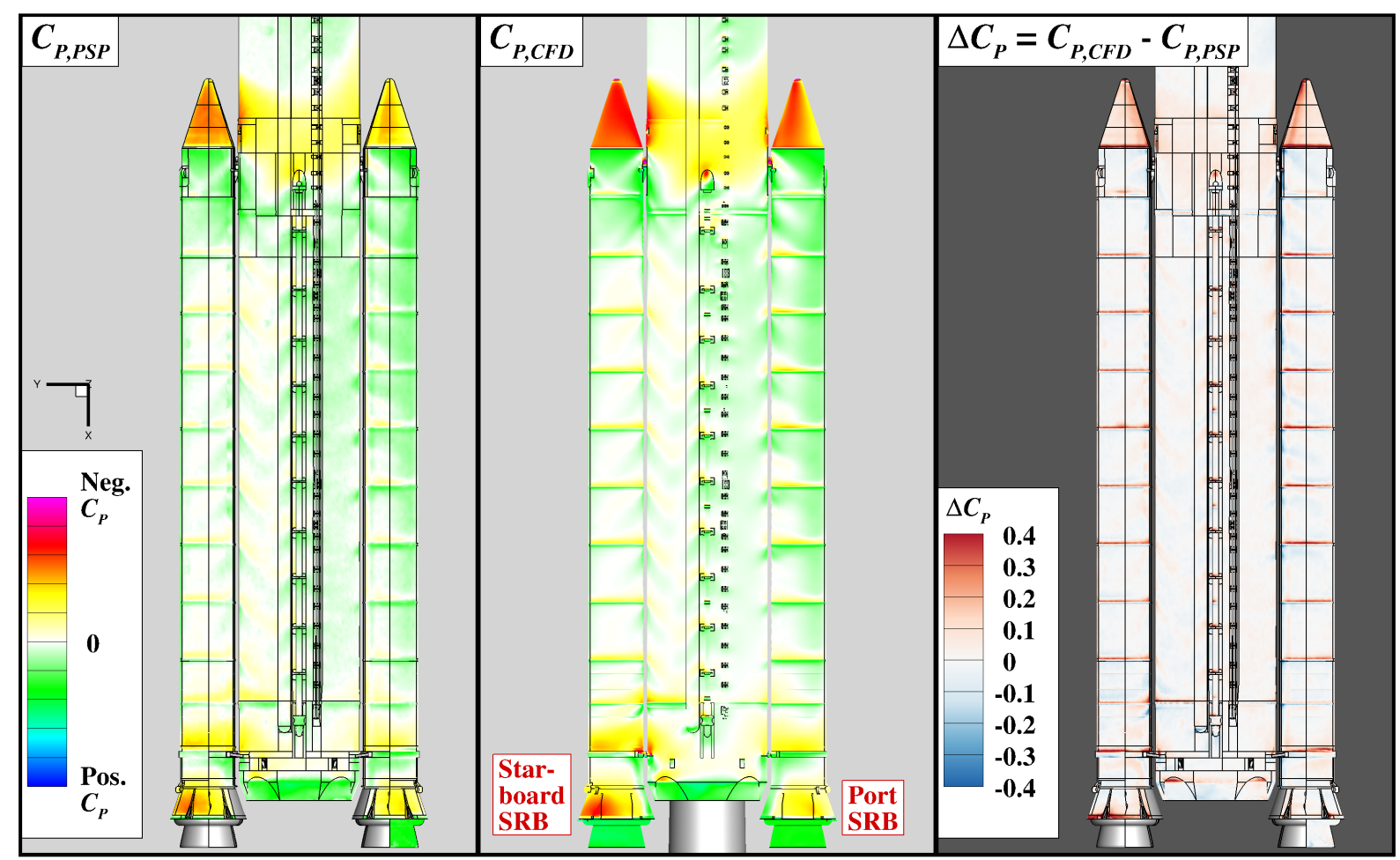

(b) $\phi_{V}=45^{\circ}$.

Fig. 32 Comparison of PSP and CFD surface pressure coefficient data on the lower surface of the core/SRBs of the SLS-28k configuration at $M_{\infty}=1.3, \alpha_{V}=8^{\circ}$, and $\phi_{V}=0^{\circ}, 45^{\circ}$, and $90^{\circ}$. 


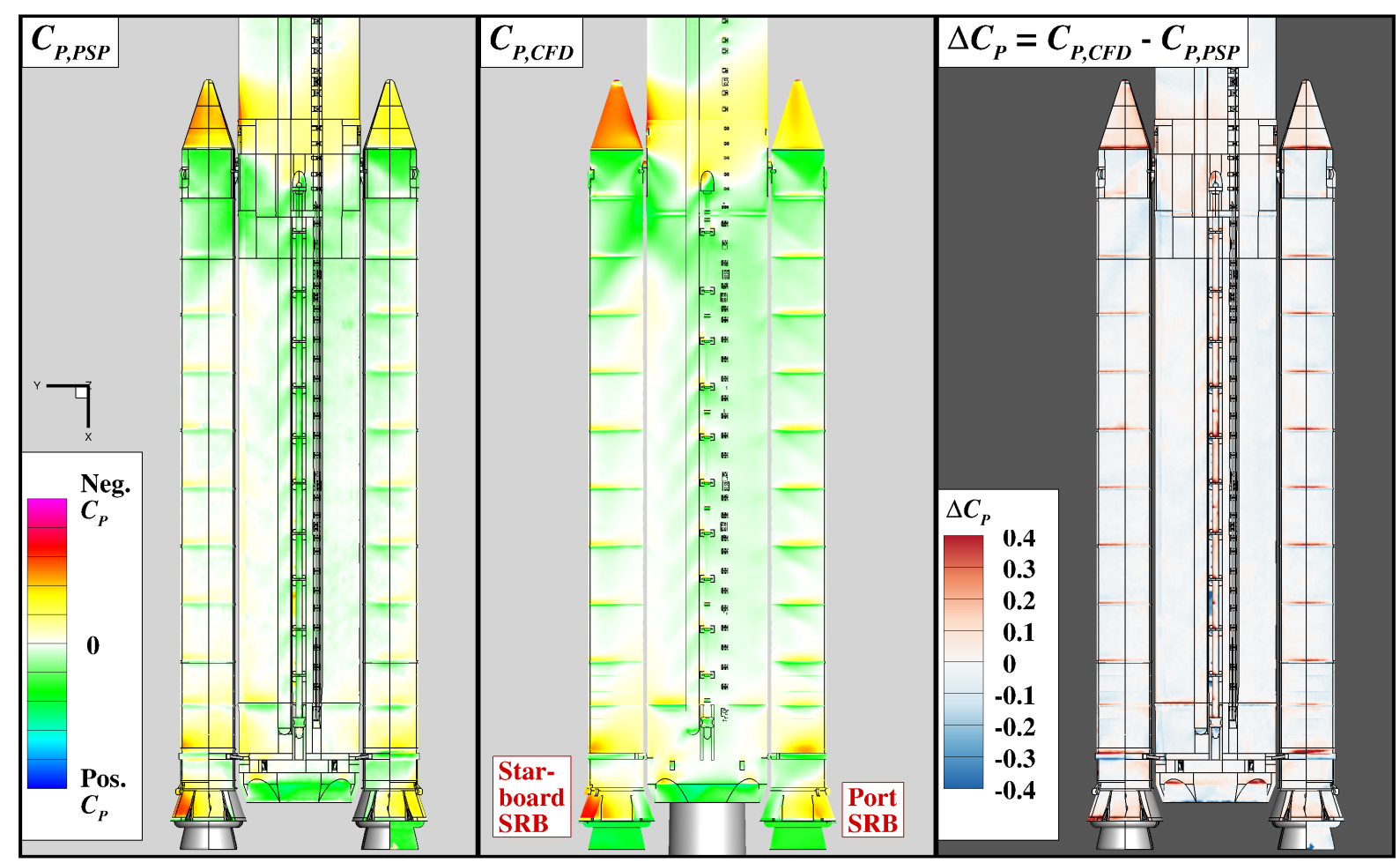

(c) $\phi_{V}=90^{\circ}$.

Fig. 32 Concluded.

This space intentionally left blank. 


\section{References}

[1] Shea, P. R., Pinier, J. T., Houlden, H. P., Favaregh, A. L., Hemsch, M. J., Dalle, D. J., Rogers, S. E., Meeroff, J. G., and Lee, H. C., "Ascent Aerodynamic Force and Moment Database Development for the Space Launch System," AIAA 2019-3298, 2019.

[2] Meeroff, J. G., Lee, H. C., Dalle, D. J., Rogers, S. E., Roozeboom, N. H., and Baerny, J. K., "Comparison of Space Launch System Sectional Loads from Pressure Sensitive Paint and CFD," AIAA 2019-2127, 2019.

[3] Holmberg, J., "NASA Ames Research Center Wind Tunnel Division, Test Planning Guide for High Speed Wind Tunnels, A027-9391-XB2, Revision 6," https://www.nasa.gov/centers/ames/pdf/643643main_HSpeedTestPlanGuide.pdf, 2005. Accessed: 1 October 2018.

[4] Kmak, F. J., "Modernization and Activation of the NASA Ames 11- by 11-Foot Transonic Wind Tunnel,” AIAA 2000-2680, 2000.

[5] Kmak, F. J., "Capabilities of the Unitary Plan Wind Tunnel," Oral Presentation, 55 ${ }^{\text {th }}$ AIAA Aerospace Sciences Mtg., 9-13 January 2017, Grapevine, TX, ARC-E-DAA-TN38585, 2017.

[6] Guide: Nomenclature and Axis Systems for Aerodynamic Wind Tunnel Testing (AIAA G-129-2012(2017)), American Institute of Aeronautics and Astronautics, Inc., 2012. doi:doi:10.2514/4.869150.001, URL https://doi .org/10.2514/4.869150.001.

[7] Roozeboom, N., and Baerny, J. K., "Guide for Pressure-Sensitive Paint Testing at NASA Ames Research Center Unitary Plan Wind Tunnel," AIAA 2017-1055, 2000.

[8] Biedron, R. T., Derlaga, J. M., Gnoffo, P. A., Hammond, D. P., Jones, W. T., Kleb, B., Lee-Rausch, E. M., Nielsen, E. J., Park, M. A., Rumsey, C. L., Thomas, J. L., , and Wood, W. A., "FUN3D Guide: 13.1," NASA TM-2017-219580, 2017.

[9] "SLS Lift Capabilities and Configurations," https://www .nasa.gov/sites/default/files/atoms/files/sls_lift_ capabilities_and_configurations_508_03152018_0.pdf, 2018. Accessed: 08 May 2018. 